\title{
2012 ACCF/AATS/SCAI/STS expert consensus document on transcatheter aortic valve replacement
}

Developed in collabration with the American Heart Association, American Society of Echocardiography, European Association for Cardio-Thoracic Surgery, Heart Failure Society of America, Mended Hearts, Society of Cardiovascular Anesthesiologists, Society of Cardiovascular Computed Tomography, and Society for Cardiovascular Magnetic Resonance

Writing David R. Holmes, Jr, MD, FACC, Chair ${ }^{*}$ Michael J. Mack, MD, FACC, Vice Chair, ${ }^{\dagger}$ Committee Sanjay Kaul, MBBS, FACC, Vice Chair,* Arvind Agnihotri, MD,

Members: $\quad$ Karen P. Alexander, MD, FACC, ${ }^{*}$ Steven R. Bailey, MD, FACC, FSCAI, ${ }^{\S}$ John H. Calhoon, MD, ${ }^{\ddagger}$ Blase A. Carabello, MD, FACC,* Milind Y. Desai, MBBS, FACC, $\|, \uparrow$

Fred H. Edwards, MD, FACC, ${ }^{\dagger}$ Gary S. Francis, MD, FACC, ${ }^{\#}$ Timothy J. Gardner, MD, FACC, ${ }^{\dagger}$ A. Pieter Kappetein, MD, PhD,** Jane A. Linderbaum, MS, CNP, AACC,* Chirojit Mukherjee, MD ${ }^{\dagger \dagger}$ Debabrata Mukherjee, MD, FACC ${ }^{*}$ Catherine M. Otto, MD, FACC, $*$ Carlos E. Ruiz, MD, PhD, FACC, FSCAI, ${ }^{\S}$ Ralph L. Sacco, MD, MS, FAHA, ${ }^{\ddagger \ddagger}$ Donnette Smith, ${ }^{\S \S}$ and James D. Thomas, MD, FACC ${ }^{\|\|}$

ACCF Task Robert A. Harrington, MD, FACC, Chair, Deepak L. Bhatt, MD, MPH, FACC, Vice Chair, Force Victor A. Ferrari, MD, FACC, John D. Fisher, MD, FACC, Mario J. Garcia, MD, FACC, Members: Timothy J. Gardner, MD, FACC, Federico Gentile, MD, FACC, Michael F. Gilson, MD, FACC, Adrian F. Hernandez, MD, FACC, Alice K. Jacobs, MD, FACC, Sanjay Kaul, MBBS, FACC, Jane A. Linderbaum, MS, CNP, AACC, David J. Moliterno, MD, FACC, and Howard H. Weitz, MD, FACC

\section{Supplemental material is available online.}

\footnotetext{
"American College of Cardiology Foundation Representative; ${ }^{\dagger}$ Society of Thoracic Surgeons Representative; ${ }^{\ddagger}$ American Association for Thoracic Surgery Representative; ${ }^{{ }^{5}}$ The Society for Cardiovascular Angiography and Interventions Representative; "Society of Cardiovascular Computed Tomography Representative; "Society for Cardiovascular Magnetic Resonance Representative; "Heart Failure Society of America Representative; ${ }^{* *}$ European Association for Cardio-Thoracic Surgery Representative; ${ }^{\dagger \dagger}$ Society of Cardiovascular Anesthesiologists Representative; ${ }^{\ddagger \ddagger}$ American Heart Association Representative; ${ }^{\S}$ Mended Hearts Consumer Advocate, Patient Representative; $\|||$ American Society of Echocardiography Representative.

Author Recusals: Writing committee members are required to recuse themselves from voting on sections to which their specific relationship with industry and other entities may apply; see Appendix 1 for recusal information.

This document was approved by the American College of Cardiology Foundation (ACCF) Board of Trustees, American Association for Thoracic Surgery (AATS) Council, Society for Cardiovascular Angiography and Interventions (SCAI) Board of Directors, Society of Thoracic Surgeons (STS) Board of Directors in January 2012 and endorsed by the governing bodies of the American Heart Association (AHA) Science Advisory and Coordinating Committee, American Society of Echocardiography (ASE), European Association for Cardio-Thoracic Surgery (EACTS), Heart Failure Society of America (HFSA), Mended Hearts, Society of Cardiovascular Anesthesiologists (SCA), Society of Cardiac Computed Tomography (SCCT), and the Society for Cardiovascular Magnetic Resonance (SCMR) in January 2012. For the purpose of complete transparency, disclosure information for the ACCF Board of Trustees, the board of the convening organization of this document, is available at: http://www.cardiosource.org/ACC/About-ACC/Leadership/ Officers-and-Trustees.aspx. ACCF board members with relevant relationships with industry to the document may review and comment on the document but may not vote on approval.
}

The American College of Cardiology Foundation requests that this document be cited as follows: Holmes DR Jr, Mack MJ, Kaul S, Agnihotri A, Alexander KP, Bailey SR, Calhoon JH, Carabello BA, Desai MY, Edwards FH, Francis GS, Gardner TJ, Kappetein AP, Linderbaum JA, Mukherjee C, Mukherjee D, Otto CM, Ruiz CE, Sacco RL, Smith D, Thomas JD. 2012 ACCF/AATS/SCAI/STS expert consensus document on transcatheter aortic valve replacement. J Thorac Cardiovasc Surg. 2012;144:e29-84.

This article has been copublished in Anesthesia and Analgesia, the Annals of Thoracic Surgery, Catheterization and Cardiovascular Interventions, and the Journal of the American College of Cardiology.

Copies: This document is available on the World Wide Web sites of the American College of Cardiology (http://www.cardiosource.org), the American Association for Thoracic Surgery (http://www.aats.org), the Society for Cardiovascular Angiography and Interventions (http://www.scai.org), the Society of Thoracic Surgeons (http://sts.org), the American Society of Echocardiography (http://www.asecho. org), the Heart Failure Society of America (http://www.hfsa.org), and Mended Hearts (http://mendedhearts.org). For copies of this document, please contact Elsevier Inc. Reprint Department, fax 212-633-3820, e-mail reprints@elsevier. com.

Permissions: Multiple copies, modification, alteration, enhancement, and/or distribution of this document are not permitted without the express permission of the American College of Cardiology Foundation. Please contact Elsevier's permission department at healthpermissions@elsevier.com. $0022-5223 / \$ 36.00$

Copyright (C) 2012 by American Association for Thoracic Surgery, the American College of Cardiology Foundation, the Society for Cardiovascular Angiography and Interventions, and the Society of Thoracic Surgeons doi:10.1016/j.jtcvs.2012.03.001 


$\begin{array}{ll}\text { Abbreviations and Acronyms } \\ \text { AR } & =\text { aortic regurgitation } \\ \text { AS } & =\text { aortic stenosis } \\ \text { AVA } & =\text { aortic valve area } \\ \text { AVR } & =\text { aortic valve replacement } \\ \text { CAD } & =\text { coronary artery disease } \\ \text { CMR } & =\text { cardiac magnetic resonance } \\ \text { COPD } & =\text { chronic obstructive pulmonary } \\ & \text { disease } \\ \text { CT } & =\text { computed tomography } \\ \text { EF } & =\text { ejection fraction } \\ \text { EOA } & =\text { effective orifice area } \\ \text { EuroSCORE }= & \text { European system for cardiac } \\ & \text { operative risk evaluation } \\ \text { LV } & =\text { left ventricular } \\ \text { LVOT } & =\text { left ventricular outflow pact } \\ \text { MDCT } & =\text { multidetector computed tomography } \\ \text { NCDR } & =\text { National Cardiovascular Data } \\ & \text { Registry } \\ \text { PARTNER } & =\text { Placement of Aortic Transcatheter } \\ & \text { Valve trial } \\ \text { PH } & =\text { pulmonary hypertension } \\ \text { RV } & =\text { right ventricular } \\ \text { SOURCE } & =\text { SAPIEN Aortic Biosprosthesis } \\ & \text { European Outcome registry } \\ \text { STS } & =\text { Society of Thoracic Surgeons } \\ \text { TAVR } & =\text { transcatheter aortic valve } \\ & \text { replacement } \\ \text { TEE } & =\text { transesophageal echocardiogram } \\ \text { TTE } & =\text { transthoracic echocardiography } \\ \text { VARC } & =\text { Valve Academic Research } \\ & \text { Consortium } \\ & \end{array}$

\section{TABLE OF CONTENTS}

Preamble ....................... e31

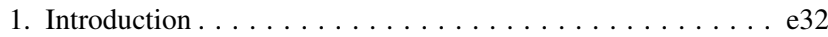

1.1. Document Development Process . . . . . . . . . e32

1.1.1. Writing Committee Organization . . . . . . . . e32

1.1.2. Document Development and Approval . . . . . . e32

1.2. Purpose of This Document . . . . . . . . . . e 32

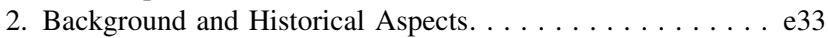

2.1. Pathophysiology and Clinical Course . . . . . . . e 33

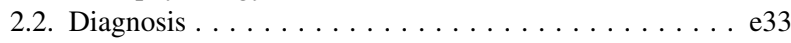
2.2.1. Echocardiography Versus Catheterization ..... e 33

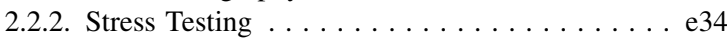

2.3. Special Considerations . . . . . . . . . . . . . e 34 2.3.1. Symptom Status. . . . . . . . . . . . . . e 34 2.3.2. Associated Coronary Artery Disease. . . . . . . e e34 2.3.3. Associated Lesions-AR, MR, Pulmonary

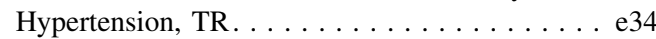

2.3.4. Low Gradient-Low EF . . . . . . . . . . . . . e 35

2.3.5. Basal Septal Hypertrophy-Outflow Tract

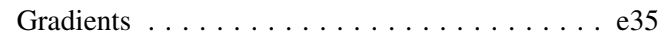

3. Current Treatment Options . . . . . . . . . . e 35

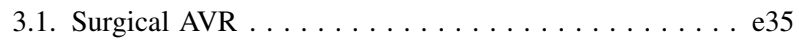

3.1.1. Valve Type ................ e35

3.1.1.1. Mechanical Valves ............. e35

3.1.1.2. Bioprosthetic Valves. . . . . . . . . e 35

3.1.2. Procedural Hazards . . . . . . . . . . e e36

3.1.3. Patient Selection . . . . . . . . . . . e36 3.1.3.1. Use of sts and euroscore Models in

Patient Selection for Conventional AVR e36

3.1.3.2. Patient Risk of AVR. . . . . . . . . . e36 3.1.3.2.1. Specific Surgical Risks. . . . e 36

3.1.3.2.1.1. Stroke...... . e36

3.1.3.2.1.2. Other

Complications .. e 37

3.1.3.3. Prohibitive Risk, Extreme Risk, Inoperability . . . . . . . . . e 37

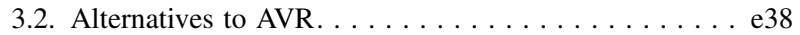

3.2.1. Medical Therapy ... . . . . . . . . . . e 38

3.2.2. Balloon Aortic Valvuloplasty. . . . . . . . . . e 38

4. Transcatheter Aortic Valve Replacement ........... e 38

4.1. Background and History. . . . . . . . . . . . e 38

4.2. Device Description ............... e 38

4.3. Current State of the Evidence . . . . . . . . . . e 40

4.3.1. Registry Experience . . . . . . . . . . . e40

4.3.1.1. Demographics . . . . . . . . . . . e 41

4.3.1.2. Outcomes . . . . . . . . . . . . e41

4.3.1.2.1. Procedural Success and Hazards . . . . . . . . . e41

4.3.1.2.2. Early and Late Morbidity and Mortality ........... e41

4.3.1.2.3. Quality of Life in Registries . e41

4.3.1.2.4. Learning Curve . . . . . . . . e e41

4.3.2. Randomized Controlled Trial. . . . . . . . . . e42

4.3.2.1. PARTNER Trial Design . . . . . . . . e42

4.3.2.2. Demographics and Other Baseline

Characteristics ............ e43

4.3.2.3. PARTNER Trial Results . . . . . . . . e e44 4.3.2.3.1. Quality of Life . . . . . . e e45 4.3.2.3.2. Continued-Access Protocol . . e45

4.3.2.4. TAVR-Specific Clinical Issues . . . . . . . e46 4.3.2.4.1. Stroke ............ e46 4.3.2.4.2. Conduction Defects ..... . . e47 4.3.2.4.3. Vascular Complications .... e e47 4.3.2.4.4. Patient Preferences. . . . . . e e47 4.3.2.4.5. Benefit/Risk Assessment. . . . e47

4.3.2.5. Medtronic CoreValve U.S. Pivotal

Trial. . . . . . . . . . . . e 48

5. Integration of TAVR Into Clinical Practice . . . . . . . . e e48

5.1. Patient Evaluation and Management . . . . . . . . . e48

5.1.1. Multidisciplinary Team . . . . . . . . . . . e48

5.1.2. Patient Selection . . . . . . . . . . . . e49 5.1.2.1. Inclusion/Exclusion Criteria. . . . . . . . e49 5.1.2.2. Specific Patient Subsets ... . . . . . e e49 5.1.2.2.1. Porcelain Aorta, Friable Aortic Atheroma, Radiation Heart Disease. . . . . . . . . . e 49

5.1.2.2.2. Very Elderly . . . . . . . . . e e49

5.1.2.2.3. Frailty and Futility Versus Utility ........... e 49

5.1.3. Care Plan in Candidates for TAVR. . . . . . . . e51

5.1.4. Imaging Assessment. . . . . . . . . . . . e 51 5.1.4.1. Echocardiography. .......... e51 
5.1.4.1.1. Annulus Size and Cusp and Root Anatomy. ........ e51

5.1.4.1.2. Aortic Root Disease and Ascending Aortic Dimensions e52

5.1.4.1.3. Three-Dimensional Echocardiography ...... e52

5.1.4.2. Tomographic Imaging. . . . . . . . . . . e 52 5.1.4.2.1. Rationale for Tomographic Imaging . . . . . . . e52

5.1.4.2.2. Multidetector Computed Tomography ......... e52

5.1.4.2.3. Cardiac Magnetic Resonance .......... e53

5.2. Procedural Performance ... . . . . . . . . . . e e54 5.2.1. Role of Surgeon and/or Cardiologist. . . . . . . e54 5.2.2. Procedural Location . . . . . . . . . . . . . . e 55

5.2.3. Anesthetic Considerations for TAVR ... . . . e e55

5.2.4. Vascular Access. . . . . . . . . . . . . . . e 57 5.2.4.1. Cardiopulmonary Bypass Requirement . e57

5.2.4.2. Percutaneous or Cutdown Access. . . . . e57

5.2.4.3. Deployment Technique ... . . . . . . e57

5.2.4.4. Balloon Expandable Versus

Self-Expanding Prostheses. . . . . . . e e58

5.2.5. Imaging During TAVR . . . . . . . . . . . e58

5.2.5.1. Recommendations for TEE Guidance for Patients Undergoing TAVR . . . . . . . e 58

5.2.5.2. Balloon Dilation and Size . . . . . . . e e59

5.2.5.3. Rapid Pacing. . . . . . . . . . . . . . e59

5.2.5.4. Experience With TAVR. . . . . . . . . e e60

5.3. Complication Management . . . . . . . . . . e e61 5.3.1. Shock, Low Cardiac Output Post-TAVR . . . . . e e61

5.3.2. Annular Rupture . . . . . . . . . . . . . . . . e61

5.3.3. Post-TAVR Aortic Regurgitation . . . . . . . . e62

5.3.4. Post-TAVR Heart Block . . . . . . . . . . . . e63

5.3.5. Post-TAVR Device Migration/Malposition. . . . . e63

5.3.6. Ventricular and Vascular Perforation. . . . . . . e63

5.4. Postprocedural Care. . . . . . . . . . . . . . e63

5.4.1. Postprocedural Recovery. . . . . . . . . . . . . e63

5.4.1.1. Recommendations for Procedural Care After TAVR. . . . . . . . . . . e63

5.4.2. Hemodynamic Assessment . . . . . . . . . . . e66

5.4.3. Atrial Fibrillation. . . . . . . . . . . . . e64

5.4.4. Treatment of Stroke . . . . . . . . . . . . e64

5.5. Long-Term Care . . . . . . . . . . . . . . . . . . . e64

5.5.1. Role of Primary Cardiologist. . . . . . . . . . e64

5.5.2. Follow-Up Visits . . . . . . . . . . . . . . e65

5.5.3. Hemodynamic Evaluation . . . . . . . . . . . e65

5.5.4. Interaction of Co-Treatments . . . . . . . . . . e65

5.5.5. Management of AR . . . . . . . . . . . e65

5.5.5.1. Recommendations for Managing Severe AR After TAVR. . . . . . . . . . . e65

6. Technology Evolution . . . . . . . . . . . . . e65

7. Applications in New Patient Populations and New

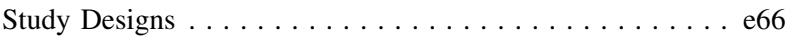

7.1. Valve-in-Valve. . . . . . . . . . . . . . . e 66

7.2. Bicuspid Aortic Valve . . . . . . . . . . . e66

7.3. Lower-Risk Populations . . . . . . . . . . . . e66

8. Introduction of TAVR Into Practice: U.S. Versus

European Perspective . . . . . . . . . . . . . . e67

8.1. U.S. Perspective . . . . . . . . . . . . . . . e67

8.2. European Perspective. . . . . . . . . . . . . e67
9. Role of Registries. . . . . . . . . . . . . . . . . . . . e67

10. Summary and Recommendations . . . . . . . . . . e69

President and Staff . . . . . . . . . . . . . . . . e 71

References..................... e71

Appendix 1. Author Relationships With Industry and Other Entities (Relevant)—2012 ACCF/AATS/SCAI/STS Expert Consensus Document on Transcatheter Aortic Valve Replacement . . . . . . . . . . . . . . e77

Appendix 2. Reviewer Relationships with Industry and Other Entities (Relevant)—2012 ACCF/AATS/SCAI/STS Expert Consensus Document on Transcatheter Aortic Valve Replacement. . . . . . . . . . . . . . e79

\section{PREAMBLE}

This document has been developed as an Expert Consensus Document (ECD) by the American College of Cardiology Foundation (ACCF), American Association for Thoracic Surgery (AATS), Society for Cardiovascular Angiography and Interventions, and the Society of Thoracic Surgeons in collaboration with the American Heart Association (AHA), American Society of Echocardiography, European Association for Cardio-Thoracic Surgery, Heart Failure Society of America, Society of Cardiovascular Computed Tomography, Society of Cardiac Magnetic Resonance, Society of Cardiovascular Anesthesiologists, and Mended Hearts. ECDs are intended to inform practitioners, payers, and other interested parties of the opinion of ACCF and document cosponsors concerning evolving areas of clinical practice and/or technologies that may be widely available or may be new to the practice community. Topics chosen for coverage by ECDs are so designed because the evidence base, the experience with technology, and/or clinical practice are not considered sufficiently well developed to be evaluated by the formal ACCF/ AHA Practice Guidelines process. Often the topic is the subject of considerable ongoing investigation. Thus, the reader should view the ECD as the best attempt of the ACCF and document cosponsors to inform and guide clinical practice in areas where rigorous evidence may not yet be available or evidence to date is not widely applied to clinical practice. When feasible, ECDs include indications or contraindications. Some topics covered by ECDs will be addressed subsequently by the ACCF/AHA Practice Guidelines Committee.

To avoid actual, potential, or perceived conflicts of interest that may arise as a result of industry relationships or personal interests among the writing committee, all members of the writing committee, as well as peer reviewers of the document, are asked to disclose all current health carerelated relationships, including those existing 12 months before initiation of the writing effort. The ACCF Task Force on Clinical Expert Consensus Documents (TF CECD) reviews these disclosures to determine what companies make products (on market or in development) that pertain to the document under development. Based on this information, a writing committee is formed to include a majority of 
members with no relevant relationships with industry or other entity (RWI), led by a chair with no relevant RWI. Authors with relevant RWI are not permitted to draft or vote on text or recommendations pertaining to their RWI. RWI is reviewed on all conference calls and updated as changes occur. Author and peer reviewer RWI pertinent to this document are disclosed in Appendices 1 and 2 , respectively. Additionally, to ensure complete transparency, authors' comprehensive health care-related disclosure information-including RWI not pertinent to this document-is available online (see Online Appendix 3). Disclosure information for the ACCF TF CECD is also available online at www.cardiosource.org/ACC/ About-ACC/Leadership/Guidelines-and-Documents-TaskForces.aspx, as well as the ACCF disclosure policy for document development at www.cardiosource.org/ScienceAnd-Quality/Practice-Guidelines-and-Quality-Standards/ Relationships-With-Industry-Policy.aspx.

The work of the writing committee was supported exclusively by the ACCF without commercial support. Writing committee members volunteered their time to this effort. Conference calls of the writing committee were confidential and attended only by committee members.

Robert A. Harrington, MD, FACC

Chair, ACCF Task Force on Clinical Expert Consensus

Documents

\section{INTRODUCTION}

\subsection{Document Development Process}

\subsubsection{Writing Committee Organization}

The Writing Committee consisted of a broad range of members representing 12 societies and the following areas of expertise: cardiothoracic surgery, interventional cardiology, general cardiology, geriatric cardiology, echocardiography, cardiac anesthesiology, cardiac computed tomography (CT), cardiac magnetic resonance (CMR), cardiac nursing, heart failure, neurology, valvular heart disease, structural heart disease, and the consumer perspective. Geographic distribution of members crossed most U.S. time zones and included international representation. Members with expertise using this new and emerging technology and those with expertise in their content area, but not in the procedure discussed herein, served on the committee to provide appropriate balance of perspectives.

This writing committee met the College's disclosure requirements for relationships with industry as described in the Preamble. Important to note, if an author works in an institution that serves as a TAVR trial site but has no direct relationship with the trial sponsor or other relevant company (that produces [competing] products or services discussed in this document) or institutional relationship as defined by the ACCF Disclosure Policy for Document
Development, the trial site information was not deemed relevant to this writing effort and is not included in the table of relevant author disclosures (Appendix 1). For example, if an author works in an institution where TAVR is performed, but he/she: (1) does not personally perform the procedure; or (2) performs the procedure but has no direct relationship to the trial (eg, principal investigator, investigator, steering committee member, consultant) and does not oversee funds related to the trial, then the relationship is not included in the table of relevant disclosures. In these situations, these relationships do not even need to be disclosed. However, in the spirit of full disclosure, this information is recorded in the online disclosure table containing all author health care relationships.

\subsubsection{Document Development and Approval}

The Writing Committee convened by conference call and e-mail to finalize the document outline, develop the initial draft, revise the draft per committee feedback, and ultimately sign off on the document for external peer review. All participating organizations participated in peer review, resulting in 48 reviewers representing 1087 comments. Comments were reviewed and addressed by the writing committee. A member of the ACCF TF CECD served as lead reviewer to ensure that all comments were addressed adequately. Both the Writing Committee and TF CECD approved the final document to be sent for board review. The ACCF Board of Trustees, AATS Council, SCAI Board of Directors, and STS Board of Directors reviewed the document, including all peer review comments and Writing Committee responses, and approved the document in January 2012. The AHA, ASE, EACTS, HFSA, Mended Hearts, SCA, SCCT, and SCMR endorsed the document in January 2012. This document is considered current until the TF CECD revises or withdraws it from publication.

\subsection{Purpose of This Document}

Transcatheter aortic valve replacement (TAVR) offers new and potentially transformational technology for patients with severe aortic valvular stenosis who are either extremely high-risk candidates or inoperable for surgical aortic valve replacement (AVR) or who are inoperable by virtue of associated comorbidities. In the future, this technology may be utilized in lower risk surgical candidates. An estimated 40,000 patients have received TAVR worldwide. Multiple single and multicenter registries, and a single randomized trial, have documented favorable outcomes using a wide spectrum of endpoints, including survival, symptom status, quality of life, and need for repeat hospitalization. The implementation of TAVR into the flow of patient care is complex, involving consideration of several key factors such as clinical site selection, operator and team training and experience, patient selection and 
evaluation, procedural performance and complication management, and postprocedural care. Collaborative stakeholder involvement is required in the management of this high-risk patient population with extensive coexistent medical conditions. A previously published document by ACCF and STS identified a high-level series of issues to be addressed regarding this technology. ${ }^{1}$ This current collaborative expert consensus document, which involves 12 professional societies, addresses these issues in greater detail with the intent to examine the current state of the evidence, facilitate the integration of this technology into the armamentarium of therapeutic options for patients with aortic valvular stenosis, and to enable responsible adoption and diffusion of this promising technology. This document has focused on published data; it must be remembered that there is only 1 single completed randomized trial, although others are in progress or planned; much of the data in this expert consensus document is based upon information from studies and registries, both surgical and TAVR, which are frequently retrospective and include self-reported clinical events rather than adjudicated events.

\section{BACKGROUND AND HISTORICAL ASPECTS}

The most common cause of valvular aortic stenosis (AS) in adults is calcification of a normal trileaflet or congenital bicuspid valve. ${ }^{2-4}$ Calcific AS is characterized by lipid accumulation, inflammation, fibrosis, and calcification ${ }^{5,6}$ and is common in the United States. It typically presents in older individuals (ie, $>75$ years) in contrast to bicuspid AS, which presents a decade or more earlier. Rheumatic AS, uncommon in the Western world, occurs due to fusion of the commissures with scarring and calcification of the cusps, and retraction of the leaflets resulting in the valve being both regurgitant and stenotic.

\subsection{Pathophysiology and Clinical Course}

In adults with valvular AS, the obstruction develops gradually, typically over many years during which the left ventricle (LV) adapts to the systolic pressure overload with progressive concentric hypertrophy that results in diastolic dysfunction, ${ }^{4,7,8}$ reduced coronary reserve,, 10 myocardial ischemia, ${ }^{11}$ and eventually, depressed contractility resulting in LV systolic dysfunction. ${ }^{12-14}$ Ultimately, in some patients, heart failure or sudden death occurs. Typically, patients with AS are free from cardiovascular symptoms (ie, angina, syncope, and heart failure) until late in the course of the disease. However, once symptoms manifest, the prognosis is poor, with the interval from the onset of symptoms to the time of death being approximately 2 years in patients with heart failure, 3 years in those with syncope, and 5 years in those with angina. ${ }^{15}$ Gardin et al. reported that among symptomatic patients with moderate-to-severe AS treated medically, mortality rates after the onset of symptoms were approximately $25 \%$ at 1 year and $50 \%$ at 2 years, ${ }^{16}$ with approximately $50 \%$ of deaths being sudden. In the elderly high-risk patients in the PARTNER (Placement of Aortic Transcatheter Valve) trial who were treated medically (Cohort B), the survival at 1 year was only $50 \%{ }^{15}$

The natural history of AS has changed since the publication of the seminal paper by Morrow and colleagues in 1968. ${ }^{17}$ The original data were derived largely from patients with rheumatic AS or AS due to a bicuspid aortic valve, with an average age of death of 63 years. On the contrary, patients being considered for TAVR on a trileaflet valve present much later in life, typically in their late 70 s or older, and have dominantly fibrocalcific AS. Although now occurring later in life, the onset of symptoms still heralds a rapid decline with medical therapy alone. ${ }^{15}$

\subsection{Diagnosis}

\subsubsection{Echocardiography Versus Catheterization}

Assessment of the severity of stenosis does not differ in TAVR patients compared with the general AS population, and decisions should therefore be based upon established guidelines. ${ }^{18}$ Although invasive cardiac catheterization has historically been the standard for quantification of AS, this function has been largely replaced by echocardiography. ${ }^{19}$

Echocardiographic diagnosis is made by the observation of a calcified valve with restricted leaflet opening by twodimensional (2D) echocardiography with quantification of the peak and mean AV gradient made by applying the simplified Bernoulli equation $\left(\Delta \mathrm{p}=4 \mathrm{v}^{2}\right)$ to the maximal velocity recorded through the aortic valve by continuous-wave Doppler. Multiple imaging windows (apical 4-chamber and long-axis, right parasternal, suprasternal notch, and subcostal views) should be obtained to assure acquisition of the maximal velocity and to avoid angle-related errors. Although aortic valve area (AVA) can be measured by planimetry, it is more accurately assessed by application of the continuity equation, using pulsed-wave Doppler in the left ventricular outflow tract (LVOT) and continuouswave Doppler across the valve. Severe stenosis is defined in the guidelines as a peak velocity $>4.0 \mathrm{~m} / \mathrm{s}$ (corresponding to a peak gradient of $64 \mathrm{~mm} \mathrm{Hg}$ ), a mean gradient $>40 \mathrm{~mm}$ $\mathrm{Hg}$, OR valve area $<1.0 \mathrm{~cm}^{2}$ when $\mathrm{LV}$ systolic function is normal. To account for patient size, the valve area is often indexed to body surface area, with $0.6 \mathrm{~cm}^{2} / \mathrm{m}^{2}$ considered to be the threshold for severe AS. An important exception is when the gradient suggests less severe stenosis than the valve area, most commonly due to low stroke volume, either in dilated ventricles with low ejection fraction (EF) or small ventricles with normal EF. In this setting, a dobutamine stress study (maximum stress dose $20 \mathrm{mcg} / \mathrm{kg} / \mathrm{min}$ ), may be helpful. If the maximum jet velocity rises over $4 \mathrm{~m} / \mathrm{s}$ with the dobutamine-induced increase in stroke volume whereas the AVA remains less than $1.0 \mathrm{~cm}^{2}$, then the valve 
is truly severely stenotic. On the other hand, if stroke volume increases with little rise in gradient (causing valve area to increase substantially), then the AS is only mild to moderate in severity, and the LV dysfunction is due to causes other than AS. ${ }^{20-22}$

Occasionally, the AVA appears larger than the elevated gradient would suggest, usually due to elevated stroke volume from aortic regurgitation (AR), anemia, fever, or hyperthyroidism. Sometimes, though, it reflects a technical error in applying the continuity equation, when the blood accelerates within the LVOT due to an upper septal bulge, which may result in an overestimation of valve area. To avoid this, one can try to measure the LVOT area at the point of maximal velocity, though the geometry is often quite distorted in this region, making estimation of the LVOT area difficult. Alternatively, one can use the LV stroke volume (from 2D or three-dimensional [3D] measurements of the LV, ideally with contrast infusion) or right ventricular (RV) stroke volume (from RV outflow tract) as the input into the continuity equation. Dividing this stroke volume by the time velocity integral of the AV continuous-wave Doppler will also yield the AVA, independent of any distortion in the LVOT.

Despite the convenience and wide-spread applicability of transthoracic echocardiography (TTE), there are occasions when invasive measurements are needed, such as in patients with a discrepancy between clinical and echocardiographic assessments. In such cases, catheterization should generally be performed with dual catheters, 1 placed in the LV, the other in the proximal aorta to obtain simultaneous pressure measurements and obtain the most accurate assessment of the gradient. Infusion of dobutamine may allow assessment of low-output, low-gradient AS in the catheterization laboratory. ${ }^{23}$ Other adjunctive testing used in quantifying AS includes transesophageal echocardiography (TEE), ${ }^{24} \mathrm{CT}$ scanning (dynamic or gated during systole), ${ }^{25}$ and CMR. ${ }^{26}$

\subsubsection{Stress Testing}

The presence or absence of symptoms should guide the management of AS patients, yet in many cases, this important clinical benchmark is difficult to establish, owing to the subjective nature of the symptoms and comorbid conditions such as chronic lung disease in this patient population. In general, stress testing is contraindicated when symptoms are present because of the potential for complications in these patients. However, in patients with equivocal symptoms, stress testing, and in particular stress echocardiography, can be very helpful. ${ }^{27}$ Simple determination of functional capacity may help show limitations of which a patient may be unaware. Isolated echocardiographic (ECG) changes during the stress test without symptoms or change in blood pressure should not be interpreted as a positive indicator of severe AS. Other potential markers for AS severity include signs of LV dysfunction on exercise echo or a rise in left atrial or right ventricular pressure. ${ }^{28,29}$

\subsection{Special Considerations}

\subsubsection{Symptom Status}

With severe, symptomatic, calcific AS, AVR is the only effective treatment that improves symptoms and prolongs survival. $^{30,31}$ These results are partly dependent on LV function. In the setting of LV dysfunction caused by afterload mismatch, survival is still improved, although improvement in LV function and resolution of symptoms might be incomplete after AVR. Age itself is a risk factor for adverse outcome, but it is not a contraindication to AVR even in the very elderly. ${ }^{32,33}$

\subsubsection{Associated Coronary Artery Disease}

In patients with moderate AS, who are undergoing coronary artery bypass graft surgery (CABG), AVR should be performed at the time of revascularization irrespective of symptoms related to moderate AS. ${ }^{34,35}$ There are no data to support performing AVR for mild AS at the time of CABG. Patients undergoing surgical AVR with significant stenoses ( $>50 \%$ to $70 \%$ stenosis) in major coronary arteries should be treated with concomitant CABG. Options in patients with combined AS and CAD continue to grow with the use of hybrid procedures where PCI is followed by valve surgery. It is possible that such a strategy could be performed in the setting of TAVR. ${ }^{36,37}$

\subsubsection{Associated Lesions-AR, MR, Pulmonary Hypertension, TR}

Patients with severe AS often have additional associated significant valvular heart disease. Treatment of these lesions in patients undergoing AVR should be undertaken using standard criteria. However, treatment of associated valvular lesions may increase the risk of AVR. ${ }^{38} \mathrm{~A}$ special circumstance is that of pulmonary hypertension $(\mathrm{PH})$ either primary or secondary (reactive or related to increased LV end-diastolic pressure). Both conditions may increase the risk of AVR and must be taken into consideration in the risk/benefit ratio.

$\mathrm{PH}$ can be present in patients with severe AS, either from the transmission of increased LV diastolic and/or left atrial pressures, associated mitral regurgitation (MR), or from a secondary increase in pulmonary vascular tone. The prevalence of $\mathrm{PH}$ in patients with AS is undefined, varying widely on the definition used and the population studied. $^{39,40}$ Clinically, PH associated with critical AS portends a poor prognosis and is associated with an increased risk of sudden cardiac death. ${ }^{41}$ Consistent with the surgical valve implant experience, $\mathrm{PH}$ after TAVR is a predictive factor for both early (30-day) and late (1year) mortality, similar in risk to major access site complications and renal insufficiency. ${ }^{39,42-46}$ The presence of $\mathrm{PH}$ makes patients more susceptible to any hemodynamic and electrical instability related to the procedure and may 
increase the risk of postprocedural complications. In addition, $\mathrm{PH}$ may result in right heart failure and severe tricuspid regurgitation (TR), both of which complicate management and increase risks.

In the setting of severe AS and PH several treatment strategies have been used. ${ }^{47}$ Persistently elevated left-sided cardiac filling pressures increase the risk of pulmonary edema when challenged with a pulmonary vasodilator. Pulmonary vasodilators, such as nitric oxide, prostacylin, and sildenafil, have been administered during and following cardiac surgery with improved hemodynamic effects. ${ }^{48-50}$ However, their overall clinical utility in improving late survival in the surgical population and their role in TAVR remains unclear. Further investigation is needed to determine the optimal procedural and periprocedural management of patients with AS and $\mathrm{PH}$ undergoing TAVR.

\subsubsection{Low Gradient-Low EF}

As mentioned, the combination of overt congestive heart failure and low aortic valve gradient is relatively common. This may be a consequence of excessive afterload (despite left ventricular hypertrophy [LVH]) or reduced contractile function $^{51}$ likely due to increased myocardial fibrosis. ${ }^{52}$ When there is overt heart failure due to low forward flow and a low transvalvular gradient (mean gradient $\leq 30 \mathrm{~mm}$ $\mathrm{Hg}$ ), both mechanisms may be present. Because of reduced contractility in the low-flow/low-gradient AS patient, prognosis with surgical AVR is adversely affected with operative mortality as high as $20 \%$. However the 5-year survival is still reported to be better in patients treated surgically. ${ }^{53,54}$ When the primary reason for poor LV performance is excessive afterload, the prognosis following surgical AVR is usually good. ${ }^{14}$ In general, patients with low gradient, low EF who have the best prognosis are those with inotropic reserve (shown by an increase in stroke volume with dobutamine infusion), who have limited coronary disease and a mean gradient that although low, still exceeds $20 \mathrm{~mm} \mathrm{Hg} .{ }^{53}$

\subsubsection{Basal Septal Hypertrophy-Outflow Tract Gradients}

Although infrequent, proximal septal bulging with LVOT obstruction may present unique issues in the presence of AS. While this can be readily addressed during AVR via myomectomy, such an approach would not be possible with TAVR. Thus, careful preprocedural echocardiographic screening is recommended to specifically avoid this scenario in patients being considered for TAVR.

\section{CURRENT TREATMENT OPTIONS}

\subsection{Surgical AVR}

AVR is the only effective treatment considered a Class I recommendation by $\mathrm{ACCF} / \mathrm{AHA}$ and $\mathrm{ESC}$ guidelines in adults with severe symptomatic AS. ${ }^{28,29}$ Not only does it offer symptomatic relief, the operation improves longterm survival. Since 1960, when AVR was first introduced, advancement in prosthetic technology including improved hemodynamics, durability and thromboresistance, and techniques in cardiac surgery such as cardioplegia, management of the small aortic root, resection of associated subvalvular disease, and replacement of associated aortic aneurysm have resulted in improvements in both operative and longterm results.

\subsubsection{Valve Type}

Current AVR options include mechanical, bioprosthetic, and in specific situations homograft and autograft techniques. Each has their advantages and drawbacks, but the trend in some centers in the recent era has been toward tissue valve replacement in a majority of patients because of improved durability and the lack of requirement for anticoagulation therapy.

\subsubsection{Mechanical Valves}

Mechanical valves are now extremely durable, have excellent hemodynamics, and are minimally thrombogenic with adequate anticoagulation. Current anticoagulation is mostly based on Vitamin $\mathrm{K}$ antagonists. Newer agents such as oral direct thrombin inhibitors and factor Xa inhibitors have been studied in other patient populations, mainly atrial fibrillation, and have been found to be associated with decreased bleeding risk and minimum drug or food interaction. ${ }^{55}$ They have not been well studied in patients with AVR. With warfarin there is a risk of serious thromboembolism of approximately $0.5 \%$ a year and a similar risk of major hemorrhage annually. ${ }^{56}$ Mechanical valves are typically preferred in younger patients given their reliable long-term durability.

\subsubsection{Bioprosthetic Valves}

Compared with mechanical valves, bioprosthetic valves do not require anticoagulation with warfarin, and thus have a lower risk of bleeding. However, long-term durability varies substantially with age for these valves. Structural valve degeneration leading to symptoms or reoperation, commonly associated with calcification of the biologic leaflets, occurs at an average of 10 to 12 years in younger patients and 15 to 18 years in older patients. Actuarial freedom from reoperation following implant of a modern bioprosthetic valves is approximately $95 \%$ at 5 years, $90 \%$ at 10 years, but drops to $70 \%$ at 15 years. ${ }^{57}$ Thus, bioprosthetic valves are generally preferred in older patients who are unlikely to tolerate bleeding risk associated with anticoagulation treatment and in whom a 15-year durability is reasonable. In patients with bioprosthetic valves, if prosthetic dysfunction occurs, TAVR may play an important role in solving the clinical issues in the future. 


\subsubsection{Procedural Hazards}

Current data from the Society of Thoracic Surgeons (STS) registry documents a mortality that is under 3\% for all patients undergoing AVR. As with any procedure, operative mortality is strongly correlated with the severity of the disease and comorbidity of patients. The operative risks can be estimated with online risk calculators from the STS (http://209.220.160.181/STSWebRiskCalc261/) and the European System for Cardiac Operative Risk Evaluation (www.euroscore.org). ${ }^{58,59}$ In selected patients with minimal comorbidity, mortality and major morbidity are under $1 \%$ each in many centers. In general, perioperative stroke rates are $1.5 \%$ (with major life-debilitating stroke being somewhat less) and other major complications are relatively rare. Renal failure, pulmonary failure, and gastrointestinal complications are not common. As older, more frail patients with extensive comorbidities undergo AVR, the risk of death and morbidity as well as length of hospitalization increases significantly. ${ }^{60,61}$ In addition to comorbidity, preoperative functional performance is also a maker of postoperative morbidity/mortality.

A recent study reviewed the results of high-risk surgical AVR in 4 centers with significant experience. The patients were a mean age of 76 and the mean STS predicted risk of mortality was $16.3 \%$. Complications included stroke in $4.4 \%$, new permanent pacemaker in $5 \%$, multisystem organ failure in $6.9 \%$, pneumonia in $7.5 \%$, and dialysis in $8.2 \%$. Postoperative length of stay was 12.6 days and inhospital mortality was $16.4 \%$. One-, 3 - and 5-year survival was $70.9 \%, 56.8 \%$, and $47.4 \%$. This study was performed between 2002 and 2007 in 4 centers before participation in the PARTNER Trial commenced and therefore serves as a reasonable baseline for comparing the results of TAVR. ${ }^{62}$

\subsubsection{Patient Selection}

Patient selection for AVR for AS is well outlined by ACCF/AHA and ESC guidelines. ${ }^{29,63}$ Problems arise when the clinicians and patients note significant symptoms and significant structural disease that are complicated by the presence of significant comorbidity. Although current STS risk score and EuroSCORE give information concerning short-term operative risks and benefits, they are not able to predict symptom resolution, quality-of-life improvement, or return to independent living.

\subsubsection{Use of STS and euroscore Models in Patient Selec- tion for Conventional AVR}

Although a number of risk algorithms for cardiac surgery have been developed, the STS and logistic EuroSCORE are the most commonly used. Although both are accurate in low-risk patients, accuracy is less in higher-risk subsets. These 2 scores include different covariates. The logistic EuroSCORE is based on 12 covariates derived from
14,799 patients undergoing all types of cardiac operations (mostly coronary bypass) in 8 European countries in 1995. On the other hand, the STS risk predictor is based on 24 covariates derived from 67,292 patients undergoing isolated AVR only in the United States over a relatively more contemporary period between 2002 and 2006. The STS model is the standard most commonly used in the United States.

\subsubsection{Patient Risk of AVR}

Information from the STS National Database shows that the operative mortality for isolated AVR has declined from $3.4 \%$ in 2002 to $2.6 \%$ today (http://www.sts. org/sites/default/files/documents/20112ndHarvestExecutive Summary.pdf). The most important preoperative patient risk factors are the need for emergency surgery, the presence of endocarditis, and a history of previous cardiac surgery. The present models do not include some risk factors that may be particularly important in the prediction of outcomes for very high-risk populations including frailty, $\mathrm{PH}$, porcelain aorta, and the presence of hepatic dysfunction, although all have been added to a recent upgraded version. ${ }^{64,65}$

It should be emphasized that risk models serve as 1 aspect of patient selection, but need to be considered in concert with clinical judgment and the other methods of risk assessment. In the final analysis, patient risk and benefit is determined, not by statistical models, but by the experience, knowledge, and expertise of the physicians charged with rendering care.

\subsection{Specific Surgical Risks}

3.1.3.2.1.1. Stroke. Although ischemic stroke can result from many causes after AVR, a major concern is the role of thromboembolism. The risks of thromboembolism are usually greater in the first few days and months after bioprosthetic AVR implantation before the sewing ring of the prosthesis is endothelialized ${ }^{66}$; risks after mechanical AVR continue. The risk of stroke within 30 days among 67,292 cases of AVR in the STS Registry was $1.5 \%$; this data set was used to develop a model for predicting 30-day stroke risk. ${ }^{61}$ Within the STS database among 108,687 AVR operations between 1996 through 2006, the risk of in-hospital permanent stroke decreased $21 \%$ from $1.7 \%$ to $1.3 \% .{ }^{67}$ It is important to note, however, that independent neurological assessment was not done in these patients, so the actual stroke incidence in these patients may be underestimated. Overall, embolic stroke risks are greater with mechanical valves, which require long-term oral anticoagulation, than with bioprosthetic valves, which have a $0.7 \%$ per year risk of thromboembolism in patients with normal sinus rhythm without warfarin anticoagulation. ${ }^{68}$

Of note, many AVR patients are older, with other comorbid cardiac conditions that increase stroke risk, including 
atrial fibrillation, cardiomyopathy, and carotid stenosis or aortic arch atheroma. ${ }^{69}$ However, even carefully selected octogenarians can safely undergo AVR with a $2 \%$ incidence of stroke. ${ }^{32,70}$

Because of the risk of stroke, the 2006 ACC/AHA guidelines for the management of patients with valvular heart disease include a variety of recommendations regarding the use of antithrombotic therapy to reduce thromboembolism risk after AVR.$^{63}$ The choice of antithrombotic agents include warfarin with target international normalized ratios (INRs) typically in the range from 2.0 to 4.0 depending on the specific prosthesis, aspirin $75 \mathrm{mg}$ to $325 \mathrm{mg}$ per day, and clopidogrel $75 \mathrm{mg}$ per day, as well as combinations. Recommendations depend upon the type of valve, timing after surgery, presence or absence of risk factors such as atrial fibrillation, and ability of the patient to take warfarin or aspirin. ${ }^{63}$

Given the greater risk of thromboembolism, particularly stroke, which usually occurs within the first 72 hours postprocedure, many centers start heparin (target aPTT $55 \mathrm{~s}$ to $70 \mathrm{~s}$ ) as soon as the risk of surgical postoperative bleeding is acceptable, which is usually within 48 hours of surgery. Heparin can be discontinued when warfarin therapy reaches a therapeutic INR usually above 2.0. ${ }^{63}$

3.1.3.2.1.2. Other Complications. Aside from other surgical complications of renal, hepatic, neurological, and pulmonary disease compromise, a major risk of conventional AVR is sternal wound infection. In most centers, this risk is under $1 \%$ for deep infection, but the risk of any type of infection is still present and particularly increased in patients with diabetes, obesity, smoking, immunosuppressive therapy, and prior radiation therapy. With the advent of negative pressure wound therapy and continued advances in surgical technique, these risks are now rarely fatal, but remain morbid. Blood requirement after valve replacement can lead to hepatitis $\mathrm{C}$, human immunodeficiency virus, or other viral infection. These transfusion-acquired infections are now extremely rare due to transfusion guidelines and systems precautions.

\subsubsection{Prohibitive Risk, Extreme Risk, Inoperability}

Despite substantial contemporary experience with successful AVR in elderly patients, multiple series have documented that $30 \%$ to $40 \%$ of patients with severe AS do not undergo surgery owing to advanced age, LV dysfunction, multiple coexisting conditions, and patient preference or physician recommendation. ${ }^{71-76}$

The definitions used to describe patient populations considered for TAVR vary; for example, prohibitive risk would describe a patient in whom the procedure could be performed from a technical standpoint but would be associated with prohibitively high morbidity and mortality. ${ }^{77}$ Inoperability might identify a patient group in whom technical success would not be possible; for example, no vascular access. Different trials have used these terms for patient enrollment; for example, the CoreValve Trial identifies extreme risk, whereas the PARTNER (Placement of AoRtic TraNscathetER Valve) Trial used the term inoperable. For this document, we prefer the term prohibitive risk. This includes some patients in whom surgery might be deemed unsuitable based on the physician's assessment of the patient's risk for surgery; whereas in others, the surgeon may decide that the operation cannot be performed successfully because of technical considerations. Assessment of inoperability is also driven by surgeon and institutional experience and thus varies. The incidence of patients undergoing AVR with an STS predicted risk of mortality $>5 \%$ is low but vary significantly amongst institutions and may be related to volume and referral patterns. Experience with such patients is pivotal for TAVR teams. Referral to such team and another opinion/consultation is crucial before deeming a patient inoperable. Whereas practice guidelines have been developed to assist physicians and surgeons in determining appropriate use of treatment options, ${ }^{29,63}$ there are, however, no specific recommendations for defining inoperability. Current ACCF/AHA guidelines acknowledge that special considerations are required for the management of advanced elderly patients with AS, since age-related and comorbid conditions commonly exist in patients in their 80s and 90s even though AVR is technically feasible even in this group. ${ }^{67,78}$

In the absence of literature evidence and guidelines recommendations, the determination of inoperability in any given patient depends on the judgment of the medical team. It is generally agreed that patients with limited life expectancy due to concurrent conditions such as malignancy, dementia, primary liver disease, chronic obstructive pulmonary disease (COPD), among others, are not appropriate for AVR. Frailty and related conditions of debility and deconditioning are known to result in inability to recover from major heart surgery such as AVR, despite operative survival and hospital discharge. ${ }^{65}$ These conditions can potentially contribute to increased surgical mortality and morbidity in the elderly. ${ }^{79}$

Inoperability from the surgeon's judgment may result from technical considerations that preclude safe performance of AVR, such as prior mediastinal irradiation, porcelain aorta or severe periannular calcification, severe aortic atheromatous disease, prior cardiac operations, among others including the internal mammary artery crossing the midline. Although infrequent, aortic valve bypass with a LV apex-to-descending aortic conduit has been used in some patients with severe AS judged to be inoperable via a mediastinal approach and cardiopulmonary bypass. ${ }^{80}$

In summary, a substantial percentage of patients with AS are judged to be inoperable for surgery based primarily on the physician's or surgeon's determination of operative risk and survivability. Although some patients may be found 
to be inoperable for technical and surgical reasons, most inoperable patients are felt to be too ill from associated comorbid conditions.

\subsection{Alternatives to AVR}

\subsubsection{Medical Therapy}

There are no proven medical treatments to prevent or delay the disease process in the aortic valve leaflets. However, evaluation and modification of cardiac risk factors is important in patients with aortic valve disease to prevent concurrent coronary artery disease (CAD). The association of AS with risk factors similar to those associated with atherosclerosis ${ }^{5,6}$ had suggested that intervention may be possible to slow or prevent disease progression in the valve leaflet, ${ }^{81,82}$ but prospective, randomized, placebocontrolled trials failed to demonstrate a benefit of statins in reducing the progression of aortic valve stenosis.

Longer-term palliative medical management of symptomatic AS may be appropriate for patients who are either not candidates for aortic valve surgery due to comorbidities or in patients who refuse AVR. The overall goal of medical therapy is to treat coexisting cardiovascular conditions, and treat superimposed diseases that often exacerbate the disease process. Patients should be educated about the effects of sodium intake, change in weight, and other factors that may lead to clinical decompensation. Medical therapy should be judicious and include treating concurrent cardiovascular conditions such as correction of anemia and fever, and preventative measures such as pneumococcal or influenza vaccination. Given the severe hypertrophy, optimizing hemodynamics by maintaining sinus rhythm may help with symptom stabilization.

Even with optimal care, adults with severe symptomatic inoperable AS will have exacerbations of symptoms and frequent hospitalizations. Palliative care should include end-of-life discussions and counseling as appropriate. Counseling is also indicated regarding true risk of AVR, and the importance of accurate risk prediction cannot be overemphasized. Many patients may refuse surgery based on misunderstood operative risk.

\subsubsection{Balloon Aortic Valvuloplasty}

First reported in $1986,{ }^{83}$ balloon aortic valvuloplasty was considered to be a less invasive and safe alternative to AVR, particularly in high surgical risk patients with multiple medical comorbidities. Although balloon aortic valvuloplasty results in immediate hemodynamic improvement with a significant decrease in transvalvular gradients resulting in larger valve area, it does not result in sustained clinical improvement because of high recurrence rates; restenosis or recoil of the aortic valve usually occurs within 6 months. Patients treated with balloon aortic valvuloplasty alone have shown poor prognosis, with survival rates of $50 \%$ at
1 year, $35 \%$ at 2 years, and $20 \%$ at 3 years. ${ }^{15,84-86}$ In addition, serious complications due to balloon aortic valvuloplasty occur in $15 \%$ to $25 \%$ of patients. ${ }^{84,87,88}$ Balloon aortic valvuloplasty, therefore, should not be used as a substitute for AVR in patients who are candidates for surgical AVR. Even as a palliative treatment, balloon aortic valvuloplasty data suggest that there is much uncertainty regarding improved longevity or quality of life after the procedure with a mean duration of symptom improvement of only 1 year. $^{63,89}$ There has been no significant difference in long-term survival demonstrated between patients undergoing balloon aortic valvuloplasty and those undergoing medical therapy alone. ${ }^{86}$ Although balloon aortic valvuloplasty as a stand-alone treatment is not recommended, ${ }^{63,87,88}$ it may still be used in contemporary practice as a bridge to subsequent AVR (both Class IIb, Level of Evidence C recommendation). ${ }^{28,84,90} \mathrm{In}$ the current era of TAVR, there has been increased interest in balloon aortic valvuloplasty. In this setting, balloon aortic valvuloplasty may be used to assess whether there is initial clinical improvement, in which case, then the patient may be a candidate for TAVR.

\section{TRANSCATHETER AORTIC VALVE REPLACEMENT}

\subsection{Background and History}

Given the increased mortality and morbidity of AVR surgery for high-risk patients and the poor long-term results of balloon aortic valvuloplasty, there has been interest in the development of a percutaneously delivered aortic heart valve. ${ }^{91}$ As early as 1992, investigators evaluated stent-based porcine bioprostheses delivered to various aortic sites in animal models. ${ }^{92}$ This early work culminated in 2000 with implantation of a percutaneous heart valve in a 12-year-old patient with a failing right ventricular to pulmonary arterial conduit that had been placed 8 years previously for the treatment of pulmonary atresia and ventricular septal defect. This initial seminal experience was followed in 2002 by the first human TAVR using the antegrade approach to implant a balloon expandable equine pericardial leaflet stent valve. ${ }^{93}$ Since that early experience, there have been multiple iterations and a number of new designs.

\subsection{Device Description}

At the present time, the most data available for TAVR are based upon 2 specific devices - the Sapien valve (Figure 1) Edwards Life Sciences, Inc, Irvine, Calif) and the CoreValve (Figure 2) (Medtronic, Inc, Minneapolis, Minn). The most recent iteration of the former is a trileaflet bovine pericardial valve mounted with a tubular slotted balloonexpandable stent composed of a cobalt chromium alloy. The Sapien valve is available in $23-\mathrm{mm}$ and $26-\mathrm{mm}$ sizes 


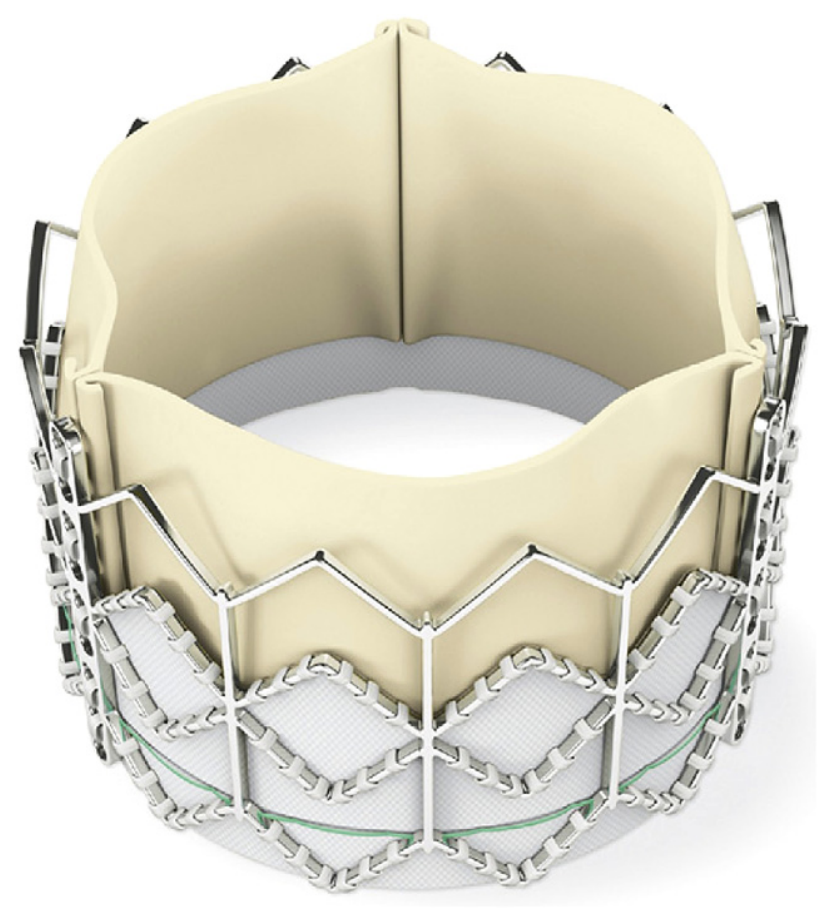

FIGURE 1. Sapien valve. Source: Edwards Lifesciences.

in the United States and 23-mm, 26- $\mathrm{mm}$, and 29-mm sizes in Europe. The initial devices required a 22- or 24-French sheath for delivery of the prosthesis. Recent iterations (NovaFlex) have decreased this to 18 -French. The first and second generations of this device have been tested in randomized controlled trials for both transfemoral and transapical implantation.

The second device (CoreValve) is comprised of 3 porcine pericardial tissue leaflets mounted in a self-expanding nitinol frame. It is available in 3 sizes- $26 \mathrm{~mm}, 29 \mathrm{~mm}$, and 31 $\mathrm{mm}$. This valve has also continued to iterate, with the initial devices being 25-French, but now 18-French delivery sheaths are used. This valve has only been used by a retrograde approach-either via transfemoral, subclavian, or direct aortic access.

A wide range of new devices has been tested with some first-in-man experiences. These devices have been characterized by smaller size, the ability to reposition or even recapture the device after deployment if an optimized device position is not obtained initially, and, modular prosthetic elements to design in situ more optimal conformance to the natural valve and aortic annulus among others.

Specific anatomic issues must be considered in device design. These include the rigid structure of the pattern of valvular calcification and aortic annulus, and the need for as full apposition as possible to the annulus in an attempt to minimize periprosthetic leak which, given sometimes eccentric, bulky calcification, may be difficult. The close proximity to the coronary ostia, the width and height of the sinuses, the membranous ventricular septum with the

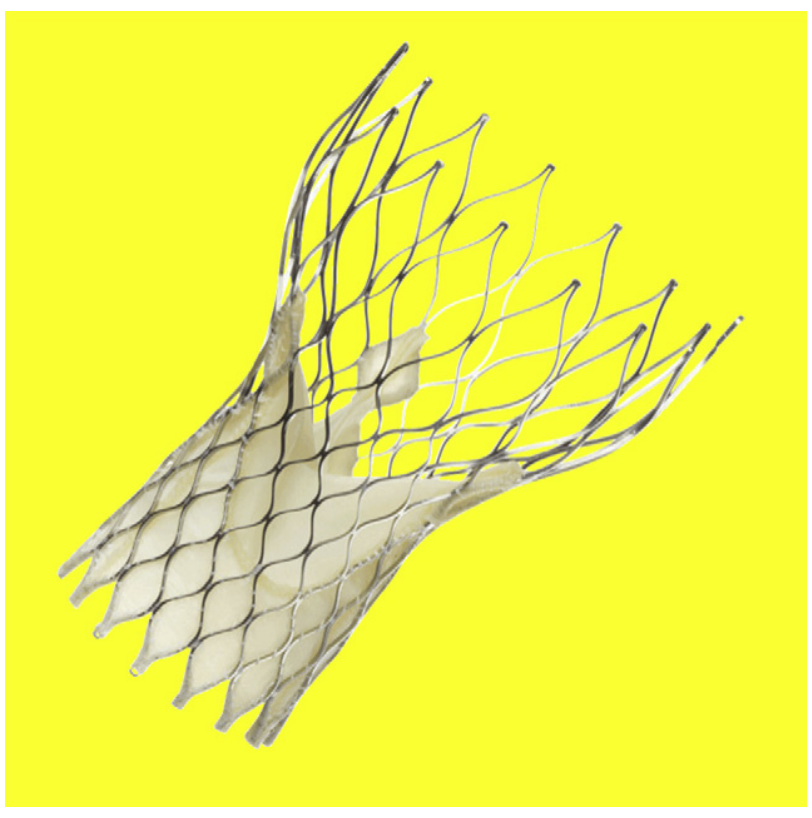

FIGURE 2. CoreValve. The Medtronic CoreValve System is currently limited to investigational use in the United States. Source: Medtronic, Inc.

His bundle and the anterior leaflet of the mitral valve are also important anatomical considerations. In addition, the size and degree of severity of peripheral arterial disease are all factors that could limit catheter size. Other issues include avoidance of central prosthetic leak, leaflet durability, hemodynamic performance, ability to treat both tricuspid and bicuspid valve anatomy, surfaces designed to minimize thrombogenicity, and the need to optimally position the devices and retrieve and reposition when necessary. ${ }^{94}$

Fundamental issues for all current and future devices are hemodynamic results, valve durability, and residual or new aortic regurgitation (AR). The initial hemodynamic performance of TAVR valves must be similar or superior to that obtained with surgical AVR. This is crucial because high residual transprosthetic gradients result in less symptomatic improvement and poorer regression of left ventricular mass. ${ }^{95}$ These transprosthetic gradients are a function of prosthetic size as well as the specific type of prosthesis and can result in patient-prosthesis mismatch. Typical immediate postprocedural gradients after surgical AVR range from $8 \mathrm{~mm} \mathrm{Hg}$ to $12 \mathrm{~mm} \mathrm{Hg}$, whereas the AV area or effective orifice area (EOA) ranges from 1.4 to $1.9 \mathrm{~cm}^{2}$. As documented below in the PARTNER trial, the valve hemodynamics of the TAVR early on are approximately $10 \%$ better than the specific surgical aortic prostheses used in that trial.

There are only limited clinical data on the durability of TAVR valves - up to 2 years-in the PARTNER trial and up to 5 years in other registry experiences. Although the absolute number of patients is small, there have been no reports of structural valve deterioration. The fundamental clinical need for durability may depend in part on the specific patient 
TABLE 1. Edwards Sapien transcatheter heart valve registries

\begin{tabular}{|c|c|c|c|c|c|}
\hline Characteristic & $\begin{array}{c}\text { REVIVE, REVIVAL, } \\
\text { PARTNER EU } \\
(\mathbf{N}=\mathbf{2 2 2}) \\
\end{array}$ & $\begin{array}{c}\text { SOURCE } \\
\text { registry (TF) } \\
(\mathbf{N}=\mathbf{9 2 0}) \\
\end{array}$ & $\begin{array}{c}\text { France registry } \\
(\mathbf{N}=1137) \\
\end{array}$ & $\begin{array}{c}\text { Belgium registry } \\
(\mathbf{N}=\mathbf{3 0 3})\end{array}$ & $\begin{array}{c}\text { Canada registry }(\mathrm{TF}) \\
(\mathrm{N}=162) \\
\end{array}$ \\
\hline \multicolumn{6}{|c|}{ Demographics } \\
\hline Age (y) & 83 & 82 & 83 & 83 & 83 \\
\hline Female $(\%)$ & 55 & 56 & 49 & 46 & 44 \\
\hline EuroSCORE (mean, \%) & 26 & 24 & 23 & 29 & 26 \\
\hline NYHA functional class III/IV (\%) & 89 & 76 & 75 & 80 & 93 \\
\hline Aortic valve area $\left(\mathrm{cm}^{2}\right)$ & 0.59 & 0.70 & 0.67 & 0.60 & 0.63 \\
\hline Mean gradient $(\mathrm{mm} \mathrm{Hg})$ & 45 & 49 & 48 & 47 & 48 \\
\hline Prior CABG $(\%)$ & 26 & 15 & 19 & 20 & 30 \\
\hline Ejection fraction $(\%)$ & 51 & 52 & 53 & 50 & 55 \\
\hline \multicolumn{6}{|c|}{ Outcomes } \\
\hline 30-day mortality (\%) & 10.4 & 7.5 & 7.8 & 8 & 9.5 \\
\hline 1-y mortality $(\%)$ & 24 & 18.9 & NR & NR & NR \\
\hline Stroke $(\%)$ & 3.3 & 3.5 & 3.5 & 5.0 & 3.0 \\
\hline Major vascular complications (\%) & 27.9 & 11.3 & 11.3 & NR & 13.1 \\
\hline Permanent pacemaker (\%) & 1.8 & 6.7 & 8.5 & 4.0 & 3.6 \\
\hline
\end{tabular}

population. In the PARTNER trial, the mean age at implant was 83 years, and serious comorbidities were frequent. In this setting, the need for durability of 20 years is less important than if the patient selection criteria are broadened to include patients in their early to mid 60s who have isolated AS without comorbid conditions. In this latter group, the TAVR valve must have at least equivalent clinical durability to currently available surgically implanted valves.

\subsection{Current State of the Evidence}

\subsubsection{Registry Experience}

Registry data provide important information for assessing the role of TAVR in a large number of patients who are not eligible for randomized controlled trials because of strict selection criteria. Several multicenter registries, including Edwards Lifesciences and Medtronic CoreValve (Tables 1 and 2), have reported early and late outcomes with TAVR. However, patient selection criteria varied amongst the different registries; standardized definitions for clinical events such as those described by the Valve Academic Research Consortium (VARC) ${ }^{96}$ were not used; and endpoints were not prospectively adjudicated using a blinded clinical event committee.

CoreValve system real-world clinical experience to date is comprised of multiple registries from several participating national sites. ${ }^{97,105-110,115}$ These study sizes range from 61 to 663 patients, with a combined clinical patient experience of nearly 2350 patients that includes follow-up of up to 2 years. (See Table 2 for details.)

TABLE 2. Medtronic CoreValve transcatheter heart valve registries

\begin{tabular}{|c|c|c|c|c|c|c|c|c|}
\hline Characteristic & $\begin{array}{c}\text { Tamburino et al }^{109} \\
(\mathrm{~N}=663)\end{array}$ & $\begin{array}{l}\text { Milan }^{107} \\
(\mathrm{~N}=61) \\
\end{array}$ & $\begin{array}{c}\text { French }^{106} \\
(\mathrm{~N}=66) \\
\end{array}$ & $\begin{array}{l}\text { Spanish }^{97} \\
(\mathrm{~N}=\mathbf{1 0 8})\end{array}$ & $\begin{array}{c}\text { UK/Ireland }^{108} \\
(\mathbf{N}=\mathbf{2 8 8})\end{array}$ & $\begin{array}{c}\mathbf{U K}^{115} \\
(\mathrm{~N}=\mathbf{4 5 2})\end{array}$ & $\begin{array}{l}\text { German }^{110} \\
(\mathrm{~N}=\mathbf{5 8 8}) \\
\end{array}$ & $\begin{array}{c}\text { Buellesfeld et al }^{105} \\
(\mathrm{~N}=126) \\
\end{array}$ \\
\hline \multicolumn{9}{|c|}{ Demographics } \\
\hline Age $(y)$ & 82 & 79 & 82.5 & 78.6 & 81 & 81.3 & 81.4 & 81.9 \\
\hline Female $(\%)$ & 56 & 47 & 51.5 & 54.6 & NR & 48 & 55.8 & 57.1 \\
\hline EuroSCORE (mean, \%) & 23 & 26.6 & 24.7 & 16 & 22 & 18.1 & 20.8 & 23.4 \\
\hline NYHA functional class III/IV (\%) & 71.5 & 69 & 74.6 & 58.4 & 74 & 73.9 & 88.2 & 74.6 \\
\hline Mean gradient (mm Hg) & 52 & 54 & 46 & 55 & NR & NR & 48.7 & 46.8 \\
\hline \multicolumn{9}{|c|}{ Outcomes } \\
\hline Procedural success $(\%)$ & 98 & 98.4 & 92.6 & 98.1 & 97.5 & 98.2 & NR & 72.6 \\
\hline 30-day mortality (\%) & 5.9 & 2.2 & 15.1 & 7.4 & 4.7 & 5.8 & 12.4 & 15.2 \\
\hline $1-y$ mortality $(\%)$ & 15 & $18.4^{*}$ & NR & 17.7 & NR & 21.7 & NR & $38.1 * *$ \\
\hline Stroke $(\%)$ & 2.5 & 2.2 & 4.5 & 0.0 & 4.2 & 4.0 & 2.8 & NR \\
\hline Major vascular complications (\%) & 2.0 & 21.3 & 7.5 & 5.6 & 9.0 & 6.2 & 4.0 & NR \\
\hline Permanent pacemaker $(\%)$ & 19.1 & 26.1 & 25.7 & 35.2 & 26 & 24.4 & 42.5 & 26.2 \\
\hline
\end{tabular}

$N$, Number; $N R$, not reported; NYHA, New York Heart Association. *6-month survival, **2-year survival. 


\subsubsection{Demographics}

Tables 1 and 2 summarize the major patient characteristics for the Sapien and CoreValve family of registries, respectively. The patients selected for entry are elderly (average age typically over 80 years), with symptomatic severe AS (mean gradient $\geq 45 \mathrm{~mm} \mathrm{Hg}$ ), significant comorbidities, and an average EuroSCORE of $\geq 23$ (Sapien) and $>16$ (CoreValve), ${ }^{97,105-110}$ indicating a significant risk with conventional AVR. However, unlike the PARTNER trial, all of these registries used the EuroSCORE risk prediction system for defining high risk and inoperability. EuroSCORE is generally not regarded as valid in high-risk patients for surgical AVR, and surgeon input as to operability was not required in these registries. As a result, the registry results are difficult to interpret because it is unclear whether the patients who were enrolled in these registries were truly "inoperable" versus "high-risk." 110,111

\subsubsection{Outcomes}

4.3.1.2.1. Procedural Success and Hazards. In the SOURCE (SAPIEN Aortic Biosprosthesis European Outcome) registry, procedural success rate (defined as 1 valve implanted, AR $<2+$, and patient left procedure room alive) was $93 \%$ for transfemoral TAVR and $92 \%$ for transapical TAVR. The procedural success rate reported for CoreValve is $>92 \%$ except for 1 study that enrolled very high-risk patients. ${ }^{105}$ Significant variations between registries were not observed in terms of deployment, relief of obstruction and avoidance of significant AR. ${ }^{110,111}$

4.3.1.2.2. Early and Late Morbidity and Mortality. The early and late major outcomes with Sapien and CoreValve registries are summarized in Tables 1 and 2. The early morbidity of TAVR includes strokes, coronary occlusion, pacemaker implantation, vascular complications, renal failure, cardiac rupture and tamponade, bleeding, aortic dissection, and death. The overall risk of any 30-day major complication ranges from $20 \%$ to over $40 \%$. Early mortality ranges from an in-hospital rate of $5 \%$ to $8 \%$ and a 30 -day mortality rate from $8 \%$ to $10 \%$. In the SOURCE registry, the incidence of a major bleeding event was significantly greater among patients undergoing transapical versus transfemoral TAVR $(3.9 \%$ vs $2.3 \%)$, whereas the incidence of vascular access-related complications was significantly higher among patients having transfemoral TAVR (major-11.3\% vs $2.0 \%$; minor- $10.4 \%$ vs $1.0 \%$ ). ${ }^{110-114}$

Permanent pacemaker placement is reported in between $1.8 \%$ up to $8.5 \%$ of patients with Sapien and $19.1 \%$ to $42.5 \%$ with the CoreValve; renal failure in under $3 \%$; and stroke in $1 \%$ to $5 \%$. Registry data reflect an overall mortality rate at 1 year of $19 \%$ to $24 \%$. In the SOURCE registry, more than half $(51.6 \%)$ of deaths up to 1 year had a noncardiac etiology and were related to baseline comorbidities. ${ }^{110,111}$
The recent UK TAVR Registry included 452 Medtronic CoreValve implantations. ${ }^{115}$ In this group, standardized data forms were used and audited. Procedural success was achieved in $98.2 \%$ in this high-risk group of patients who had a baseline logistic EuroSCORE of $18.1 \%$. Thirty-day mortality was $5.8 \%$, and 1 - and 2-year mortality was $21.7 \%$ and $23.9 \%$, respectively. In-hospital stroke occurred in $4 \%$ of patients and myocardial infarction in $1.1 \%$. A permanent pacemaker was required in $24.4 \%$ (compared with $7.4 \%$ with Sapien). Rates of moderate to severe postimplant AR were $17.3 \%$ (compared with $9.6 \%$ with Sapien). Mortality rates at all time points were significantly lower among patients treated via a transfemoral route as compared with nontransfemoral routes ( $>85 \%$ transapical). In this study, LV function, the presence of moderate/severe AR, and COPD, but not vascular access site, were independent predictors of mortality.

4.3.1.2.3. Quality of Life in Registries. Quality of life is a key patient-centered outcome. Although death is the lowest possible functional status, for many, survival marked by reduced physical function or independence may be worse than death. The PARTNER EU Registry is a multicenter study of the early European experience in TAVR. Patients undergoing TAVR by transapical or transfemoral approach were followed to 12 months for symptoms by New York Heart Association (NYHA) functional class, and heart failure-related quality of life as assessed by the Kansas City Cardiomyopathy Questionnaire. ${ }^{116}$ All patients improved, with no significant differences in NYHA functional class improvement noted between transapical or transfemoral approaches.

Several single-center registries have added additional information on quality of life using disease-specific or general surveys (Short Form-36 Health Questionnaire, Short Form-12 Health Questionnaire, Kansas City Cardiomyopathy Questionnaire, Minnesota Living with Heart Failure Questionnaire) and on symptoms (NYHA functional class, and 6-minute walk). Improvements following TAVR in vitality, physical functioning, and general and mental health scores have been identified with physical function demonstrating the greatest improvement. Patients who do not experience improvement are more likely to have comorbidities that contribute to continued symptoms and impair quality of life, such as COPD and reduced EF (Table 3).

4.3.1.2.4. Learning Curve. Each registry has identified a procedural learning curve, but the exact definition of this curve and a clear method to decrease it are not yet clearly reported. This curve has important components such as patient selection, anesthesia, improvement in the equipment over time, and technical decision making regarding valve deployment. The SOURCE registry enrolled 1038 
TABLE 3. Quality of life and symptom assessment in TAVR registries

\begin{tabular}{|c|c|c|c|c|}
\hline Study population & NYHA functional class & 6-Minute walk & Questionnaire & Other \\
\hline $\begin{array}{l}\text { PARTNER EU Registry; } \\
\text { Lefevre et al (multicenter; } \\
\mathrm{N}=130 \text { Sapien) }\end{array}$ & $\begin{array}{l}\text { Improved class at } 1 \text { year in } \\
84.5 \% \text { of patients ( } 85 \% \\
\text { NYHA functional class III/ } \\
\text { IV at baseline, } 15 \% \text { NYHA } \\
\text { functional class at } 1 \text { year); } \\
\text { changes noted at } 30 \text { days } \\
\text { were sustained }\end{array}$ & NR & $\begin{array}{l}\text { KCCQ improvement at } 1 \text { year } \\
\text { in } 72.7 \%(P<.0002)\end{array}$ & $\begin{array}{l}\text { Small improvement in EQ-5D } \\
\text { was not significant }\end{array}$ \\
\hline $\begin{array}{l}\text { Buellesfeld et al } \\
\qquad \text { (multicenter; } \mathrm{N}=126 \\
\text { CoreValve) }^{105}\end{array}$ & $\begin{array}{l}\text { Improved in } 80 \% \text { at } 30 \text { days; } \\
74 \% \text { at } 2 \text { years (in } 50 \% \text { by } \\
1 \text { level, in } 20 \% \text { by } 2 \text { or } \\
\text { more levels) }\end{array}$ & NR & NR & NR \\
\hline $\begin{array}{l}\text { Krane et al (single-center } \\
\quad \text { registry; } N=99 \text { TAVR) }\end{array}$ & $\begin{array}{l}\text { More class I/II at } 3 \text { months } \\
\text { (NYHA functional class } \\
\text { III/IV from } 98 \% \text { to } 2 \% \text { at } 3 \\
\text { months) }\end{array}$ & NR & $\begin{array}{l}\text { Improved SF-36 PF general } \\
\text { health and vitality pre/post } \\
\text { at } 3 \text { months (all } P<.01 \text { ). } \\
\text { No change mental health. }\end{array}$ & $85 \%$ would do TAVR again \\
\hline $\begin{array}{l}\text { Ussia et al (single-center } \\
\quad \text { registry; } \mathrm{N}=57 \text { TAVR) }{ }^{118}\end{array}$ & $\begin{array}{l}\text { More class I/II (average } 1.8 \\
\text { NYHA functional class } \\
\text { improvement) at } 5 \text { months } \\
(P<.001)\end{array}$ & NR & $\begin{array}{l}\text { SF-12; Improved }(P<.001) \\
\text { physical and mental } \\
\text { component scores, return to } \\
\text { population norms, greatest } \\
\text { change in PF }\end{array}$ & NR \\
\hline $\begin{array}{l}\text { Bekeredjian et al (single- } \\
\text { center registry; } \mathrm{N}=87 \\
\text { TAVR) }\end{array}$ & $\begin{array}{l}\text { Improved class (average of } \\
1.7 \text { NYHA functional class } \\
\text { improvement) at } 6 \text { months } \\
(P<.001)\end{array}$ & NR & $\begin{array}{l}\text { SF-36 Improved physical and } \\
\text { mental component scores, } \\
\text { greatest change in PF }\end{array}$ & $\begin{array}{l}70 \% \text { average decrease in } \\
\text { NT-proBNP levels of } 4000 \\
\text { ng/L }(P<.0001)\end{array}$ \\
\hline $\begin{array}{l}\text { Gotzmann et al (single-center } \\
\quad \text { registry; } \mathrm{N}=44 \text { TAVR) }\end{array}$ & $\begin{array}{l}\text { Decrease of percentage of } \\
\text { NYHA functional class III/ } \\
\text { IV from } 90 \% \text { to } 16 \% \text { at } 30 \\
\text { days }\end{array}$ & $\begin{array}{l}\text { Improved walk time } \\
\text { at } 30 \text { days }\end{array}$ & $\begin{array}{l}\text { MLHFQ; Improved HF- } \\
\text { related QOL }\end{array}$ & $\begin{array}{l}\text { Lower average decrease in } \\
\text { BNP levels of } 400 \mathrm{pg} / \mathrm{mL} \\
(P<.005) \text { and } 25 \% \\
\text { increase in 6-minute walk } \\
\text { time }(P<.005)\end{array}$ \\
\hline
\end{tabular}

$B N P$, Brain natriuretic peptide; $E Q-5 D$, EuroQol Five Dimensions; $H F$, heart failure; $K C C Q$, Kansas City Cardiomyopathy Questionnaire; $L O S$, length of stay; $M L H F Q$, Minnesota Living with Heart Failure Questionnaire; $N R$, not reported; $N Y H A$, New York Heart Association; $P F$, physical function; $Q O L$, quality of life; $S A V R$, surgical aortic valve replacement; $S F-12$, Short Form 12 Health Questionnaire; $S F$-36, Short Form 36 Health Questionnaire; TAVR, transcatheter aortic valve replacement.

(Cohort 1) and 1306 patients (Cohort 2) undergoing TAVR procedures over 2 sequential years. Age and EuroSCORE were not significantly different between the 2 cohorts. Compared with the first year of experience, valve malposition $(1.6 \%$ vs $1.2 \%)$, and vascular access complications $(2.1 \%$ vs $1.8 \%)$ were not significantly lower in the second year. However, reductions in the rates of postprocedure AR $>2+(4.5 \%$ vs $2.1 \%, P=.011)$ and conversion to open surgery $(3.7 \%$ vs $1.5 \%, P=.0315)$ were improved. ${ }^{110,111,121}$ Overall 30-day and 1-year survival was similar in both cohorts despite higher number of patients with heart failure and mitral regurgitation enrolled in Cohort 2.

In summary, the registries demonstrate in high-risk patients that TAVR may be deployed with a high degree of procedural success, predictable risk of stroke, devicedependent high risk of pacemaker implantation (particularly with CoreValve), and a 30-day mortality rate that seems potentially acceptable in a debilitated and ill patient population. Importantly, TAVR seems to alleviate AS to a similar degree as surgical AVR and patients tend to return to Class I or II symptoms with substantial improvements in quality of life.

Future registries should be designed to include contemporary (ie, VARC) definitions of procedural and qualityof-life outcomes and utilize an independent clinical events committee when possible to standardize event reporting. Longer-term follow-up studies are needed to demonstrate the continued durability of TAVR in the high-risk and inoperable patients.

\subsubsection{Randomized Controlled Trial}

\subsubsection{PARTNER Trial Design}

The PARTNER trial (Figure 3) was a prospective, unblinded, randomized, controlled, multicenter pivotal trial evaluating the safety and effectiveness of the Edwards Sapien THV transcatheter aortic valve; 2 distinct populations were enrolled-inoperable, or Cohort B, and high-risk operable, or Cohort A. Potential candidates were presented on a national conference call for approval for treatment. Randomization was stratified based on operability for 


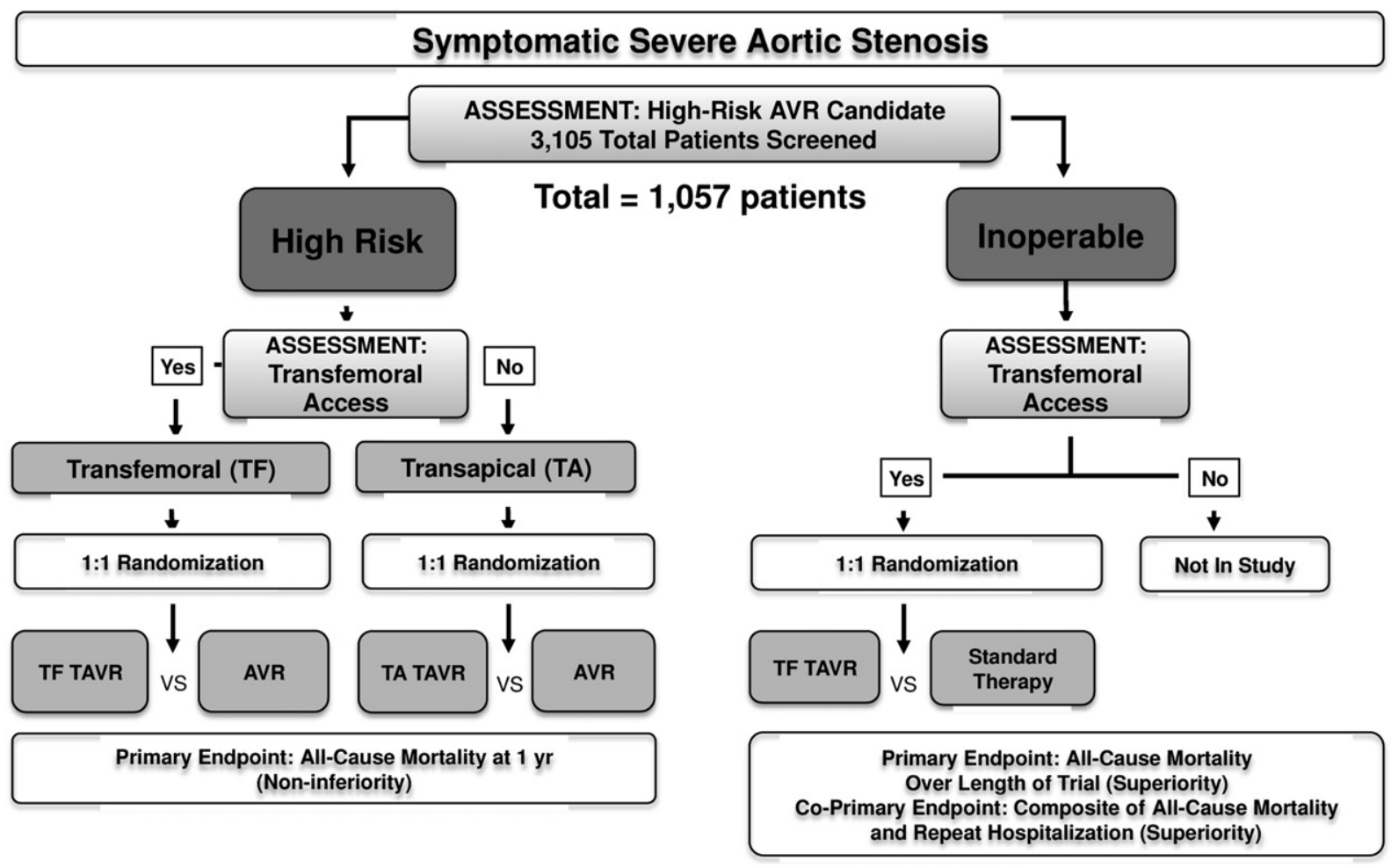

FIGURE 3. PARTNER trial design.

AVR surgery and within cohorts by vascular access for transfemoral delivery. Patients who were considered high surgical risk and eligible for transfemoral access were stratified into Cohort $\mathrm{A}$ and randomized to treatment (transfemoral AVR) or control (surgical AVR). Cohort A patients who were not eligible for transfemoral access were evaluated as candidates for transapical delivery and, if appropriate, randomized to treatment (transapical AVR) or control (surgical AVR). Nonsurgical candidates were stratified into Cohort B and randomized to treatment (transfemoral AVR) or control ("standard" therapy). Inoperability was formally defined as " $>50 \%$ predicted probability of mortality or serious irreversible complication by 30 days by 1 cardiologist and 2 cardiothoracic surgeons." ${ }^{15}$ Cohort B patients who did not meet the criteria for transfemoral delivery were not enrolled in the study because transapical delivery was deemed too risky in Cohort B (Figure 3). Of the 3105 patients screened, a total of 1057 subjects $(34 \%)$ were enrolled at 25 sites in 2 arms - 699 patients in Cohort A and 358 patients in Cohort B. There were 2 co-primary endpoints for the inoperable cohort: (1) freedom from death over the duration of the trial with all patients followed for at least 1 year from randomization; and (2) hierarchical composite of death and recurrent hospitalization. In the high-risk cohort, the primary endpoint was freedom from all-cause death at 1 year. Prespecified secondary endpoints included rate of death from cardiovascular causes, NYHA functional class, the rate of repeat hospitalization due to valve-related or procedural-related clinical deterioration, the distance covered during a 6-minute walk test, valve performance (assessed by echocardiography), and the rates of myocardial infarction, stroke, acute kidney injury, vascular complications, and bleeding. All patients were followed during the index hospitalization; at 30 days, 6 months, and 1 year; and yearly thereafter.

\subsubsection{Demographics and Other Baseline Characteristics}

The mean age was about 83 years in Cohort B and 84 in Cohort A; slightly more patients were female $(53.6 \%)$ in Cohort $\mathrm{B}$, and slightly more patients were male $(57.2 \%)$ in Cohort A; and most were Caucasian (Table 4). Over $92 \%$ in both cohorts were NYHA functional class III or $\mathrm{IV}$, and $60 \%$ of patients in both cohorts had undergone prior CABG or PCI. Overall, the groups were balanced in most baseline characteristics in Cohort A; however, there were some imbalances in Cohort B. ${ }^{15}$ Patients in both cohorts had relatively preserved LV systolic function.

Patients in Cohort B had greater frequency of coexisting conditions that contributed to the surgeons' determination of inoperability, including an extensively calcified (porcelain) aorta $(15.1 \%)$, chest-wall deformity or prior chestwall irradiation $(13.1 \%)$, oxygen-dependent respiratory insufficiency $(23.5 \%)$, and frailty, according to prespecified criteria $(23.1 \%)$. 
TABLE 4. Demographic and other baseline characteristics of the PARTNER trial (Cohort B data first)

\begin{tabular}{|c|c|c|c|c|c|c|}
\hline \multirow[b]{2}{*}{ Characteristic } & \multicolumn{3}{|c|}{ Cohort B } & \multicolumn{3}{|c|}{ Cohort A } \\
\hline & TAVR $(\mathbf{N}=\mathbf{1 7 9})$ & Standard Rx $(N=179)$ & $P$ value & $\operatorname{TAVR}(\mathbf{N}=348)$ & $\operatorname{AVR}(\mathbf{N}=351)$ & $P$ value \\
\hline \multicolumn{7}{|c|}{ Demographics } \\
\hline Age (y) & $83.1 \pm 8.6$ & $83.2 \pm 8.3$ & .95 & $83.6 \pm 6.8$ & $84.5 \pm 6.4$ & .07 \\
\hline Male $(\%)$ & 45.8 & 46.9 & .92 & 57.8 & 56.7 & .82 \\
\hline STS score & $11.2 \pm 5.8$ & $11.9 \pm 4.8$ & .21 & $11.8 \pm 3.3$ & $11.7 \pm 3.5$ & .61 \\
\hline Logistic EuroSCORE & $26.4 \pm 17.2$ & $30.4 \pm 19.1$ & .04 & $29.3 \pm 16.5$ & $29.2 \pm 15.6$ & .93 \\
\hline NYHA functional class III or IV (\%) & 92.2 & 93.9 & .68 & 94.3 & 94.0 & .79 \\
\hline $\mathrm{O}_{2}$-dependent COPD (\%) & 21.2 & 25.7 & .38 & 9.2 & 7.1 & .34 \\
\hline Frailty $(\%)$ & 18.1 & 28 & .09 & 15.6 & 17.6 & .58 \\
\hline Porcelain aorta $(\%)$ & 19 & 11.2 & .05 & 0.6 & 1.1 & .69 \\
\hline Chest wall radiation $(\%)$ & 8.9 & 8.4 & 1.00 & 0.9 & 0.9 & 1.00 \\
\hline Chest wall deformity $(\%)$ & 8.4 & 5.0 & .29 & 0 & 0.3 & 1.00 \\
\hline \multicolumn{7}{|c|}{ Echocardiographic characteristics } \\
\hline $\mathrm{AV}$ area $\left(\mathrm{cm}^{2}\right)$ & $0.6 \pm 0.2$ & $0.6 \pm 0.2$ & .97 & $0.7 \pm 0.2$ & $0.6 \pm 0.2$ & .13 \\
\hline Mean AV gradient (mm Hg) & $44.5 \pm 15.7$ & $43.0 \pm 15.3$ & .39 & $42.7 \pm 14.6$ & $43.5 \pm 14.3$ & .45 \\
\hline Mean LV EF (\%) & $53.9 \pm 13.1$ & $51.1 \pm 14.3$ & .06 & $52.5 \pm 13.5$ & $53.3 \pm 12.8$ & .45 \\
\hline
\end{tabular}

Cohort B includes only nonsurgical candidates in whom "inoperability" was formally defined as greater than 50\% predicted probability of mortality or serious irreversible complication by 30 days by 1 cardiologist and 2 cardiothoracic surgeons. Cohort A includes patients determined to be at high operative risk defined as predicted operative mortality of $\geq 15 \%$ and/or an STS risk score of $\leq 10 \%$. The STS risk algorithm is based on the presence of coexisting illnesses in order to predict 30 -day operative mortality. Data are derived from the Edwards Lifesciences' briefing document for the U.S. FDA Circulatory Devices Advisory Panel meeting on TAVR on July 21, 2011 (http://www.accessdata.fda.gov/ cdrh_docs/pdf10/P100041b.pdf) and may show some discrepancies compared with the published manuscripts. $A V$, Aortic valve; $A V R$, aortic valve replacement; $L V E F$, left ventricular ejection fraction; NYHA, New York Heart Association; $R x$, therapy; STS, Society of Thoracic Surgeons; TAVR, transcatheter aortic valve replacement.

\subsubsection{PARTNER Trial Results}

In the inoperable Cohort B patients with symptomatic severe AS, TAVR substantially reduced all-cause mortality by nearly $50 \%$ and the composite of all-cause mortality and repeat hospitalization by $55 \%$ compared with standard therapy at 1-year follow-up (Table 5). In addition, all key secondary endpoints including patient function significantly improved at 30 days and 1 year. TAVR was associated with an increased risk for stroke and procedure-related adverse events such as bleeding and vascular complications. Sensitivity analyses of patients as they were treated all favored TAVR. Overall, the benefit from TAVR in inoperable patients with symptomatic severe AS greatly exceeds the risk.

In the high-risk Cohort A patients, TAVR was noninferior to AVR for all-cause mortality at 1 year $(24.2 \%$ vs $26.8 \%$, hazard ratio: $0.93,95 \%$ confidence interval: 0.71 to 1.22 , $P=.001$ for noninferiority) (Table 6). AVR mortality at 30 days $(6.5 \%)$ was lower than expected operative mortality $(11.8 \%)$. Whether this discrepancy can be attributed to chance alone (ideal outcomes with expert surgeons within the idealized environment of a randomized trial) or due to

TABLE 5. Major outcomes at 30 days and 1 year in Cohort $B$ of the PARTNER trial

\begin{tabular}{|c|c|c|c|c|c|c|}
\hline \multirow[b]{2}{*}{ Characteristic } & \multicolumn{3}{|c|}{30 days } & \multicolumn{3}{|c|}{1 year } \\
\hline & $\begin{array}{c}\text { TAVR } \\
(\mathbf{N}=\mathbf{1 7 9}) \\
\end{array}$ & $\begin{array}{c}\text { Standard Rx } \\
(\mathbf{N}=179)\end{array}$ & $P$ value & $\begin{array}{c}\text { TAVR } \\
(\mathbf{N}=179) \\
\end{array}$ & $\begin{array}{c}\text { Standard Rx } \\
(\mathbf{N}=179)\end{array}$ & $P$ value \\
\hline All-cause death $(\%)$ & 5.0 & 2.8 & .41 & 30.7 & 49.7 & $<.001$ \\
\hline All-cause death or rehospitalization (\%) & 11.2 & 12.3 & .74 & 43.6 & 70.4 & $<.001$ \\
\hline Event-free MACCE (\%) & 90.5 & 94.4 & NR & 65.4 & 47.1 & .003 \\
\hline All stroke $(\%)$ & 7.3 & 1.7 & .02 & 11.2 & 4.5 & .03 \\
\hline Major stroke $(\%)$ & 5.6 & 1.1 & .04 & 8.4 & 3.9 & .12 \\
\hline All-cause death or major stroke $(\%)^{*}$ & 8.4 & 3.9 & .12 & 33.0 & 50.3 & .001 \\
\hline Major vascular complications (\%) & 16.8 & 1.1 & $<.0001$ & 17.3 & 2.2 & $<.0001$ \\
\hline Major bleeding ( $\%)$ & 20.6 & 3.9 & $<.0001$ & 28.4 & 14.4 & $<.001$ \\
\hline Pacemaker insertion $(\%)$ & 3.4 & 5.0 & 60 & 4.5 & 7.8 & .27 \\
\hline \multicolumn{7}{|c|}{ Echocardiographic endpoints } \\
\hline $\mathrm{AV}$ area $(\mathrm{EOA})\left(\mathrm{cm}^{2}\right)$ & $1.5 \pm 0.4$ & $0.8 \pm 0.2$ & $<.0001$ & $1.6 \pm 0.5$ & $0.7 \pm 0.32$ & $<.0001$ \\
\hline Mean AV gradient $(\mathrm{mm} \mathrm{Hg})$ & $11.1 \pm 6.6$ & $33.0 \pm 12.5$ & $<.0001$ & $12.5 \pm 10.3$ & $44.4 \pm 15.7$ & $<.0001$ \\
\hline
\end{tabular}

Cohort B includes only nonsurgical candidates in whom "inoperability" was formally defined as greater than $50 \%$ predicted probability of mortality or serious irreversible complication by 30 days by 1 cardiologist and 2 cardiothoracic surgeons. Data are based on Edwards Lifesciences' briefing document for the U.S. FDA Circulatory Devices Advisory Panel meeting on TAVR on July 21, 2011 (http://www.accessdata.fda.gov/cdrh_docs/pdf10/P100041b.pdf), and may show some discrepancies compared with the published manuscripts. AV, Aortic valve; EOA, effective orifice area; $M A C C E$, major adverse cardiac and cerebrovascular events; $N R$, not reported; $R x$, therapy; TAVR, transcatheter aortic valve replacement. *All-cause death or major stroke was not a predefined endpoint. 
TABLE 6. Major outcomes at 30 days and 1 year in Cohort A of the PARTNER trial

\begin{tabular}{|c|c|c|c|c|c|c|}
\hline \multirow[b]{2}{*}{ Characteristic } & \multicolumn{3}{|c|}{30 Days } & \multicolumn{3}{|c|}{1 Year } \\
\hline & $\begin{array}{c}\text { TAVR } \\
(\mathbf{N}=\mathbf{3 4 8})\end{array}$ & $\begin{array}{c}\text { Surgical AVR } \\
(\mathbf{N}=\mathbf{3 5 1})\end{array}$ & $P$ value & $\begin{array}{c}\text { TAVR } \\
(\mathbf{N}=\mathbf{3 4 8})\end{array}$ & $\begin{array}{c}\text { Surgical AVR } \\
(\mathbf{N}=\mathbf{3 5 1})\end{array}$ & $P$ value \\
\hline \multicolumn{7}{|c|}{ Clinical outcomes } \\
\hline All-cause death $(\%)$ & 3.4 & 6.5 & .07 & 24.2 & 26.8 & .44 \\
\hline All-cause death or rehospitalization (\%) & 7.2 & 9.7 & .24 & 34.6 & 35.9 & .73 \\
\hline All stroke $(\%)$ & 5.5 & 2.4 & .04 & 8.3 & 4.3 & .04 \\
\hline Major stroke (\%) & 3.8 & 2.1 & .20 & 5.1 & 2.4 & .07 \\
\hline All-cause death or major stroke $(\%)^{*}$ & 6.9 & 8.2 & .52 & 26.5 & 28.0 & .68 \\
\hline Major vascular complications (\%) & 17.0 & 3.8 & $<.01$ & 18.0 & 4.8 & $<.01$ \\
\hline Major bleeding $(\%)$ & 9.3 & 19.5 & $<.01$ & 14.7 & 25.7 & $<.01$ \\
\hline Atrial fibrillation (\%) & 8.6 & 16.0 & $<.01$ & 12.1 & 17.1 & .07 \\
\hline Pacemaker insertion $(\%)$ & 3.8 & 3.6 & .89 & 5.7 & 5.0 & .68 \\
\hline \multicolumn{7}{|c|}{ Echocardiographic endpoints } \\
\hline $\mathrm{AV}$ area $(\mathrm{EOA})\left(\mathrm{cm}^{2}\right)$ & $1.7 \pm 0.5$ & $1.5 \pm 0.4$ & .001 & $1.6 \pm 0.5$ & $1.4 \pm 0.5$ & .002 \\
\hline Mean AV gradient $(\mathrm{mm} \mathrm{Hg})$ & $9.9 \pm 4.8$ & $1.8 \pm 5.0$ & .16 & $10.2 \pm 4.3$ & $11.5 \pm 5.4$ & .008 \\
\hline
\end{tabular}

Cohort A includes patients determined to be at high operative risk defined as predicted operative mortality of $\geq 15 \%$ and/or an STS risk score of $\geq 10 \%$. The STS risk algorithm is based on the presence of coexisting illnesses in order to predict 30-day operative mortality. AV, Aortic valve; $A V R$, aortic valve replacement; EOA, effective orifice area; TAVR, transcatheter aortic valve replacement. *All-cause death or major stroke was not a predefined endpoint.

"calibration drift" as surgical outcomes improve over time is not clear. All neurological events (30-day major stroke, $3.8 \%$ vs $2.1 \%$ ) and vascular complications (30-day, $11.1 \%$ vs $3.2 \%$ ) were more frequent with TAVR. By contrast, major bleeding and new-onset atrial fibrillation were more frequent with AVR. Improvements in echocardiographic findings were similar in both groups, although paravalvular regurgitation was increased with TAVR. The data from this cohort further support TAVR as an acceptable alternative to surgical AVR in selected high-risk operable patients.

Of note, the 30-day mortality (generally thought to be procedure-related) in Cohort A (3.4\%) and Cohort B $(5.0 \%)$ was lower than the published SOURCE registry mortality $(8.5 \%)$, despite a relatively lower-risk patient population enrolled in the latter (1-year mortality of $30.7 \%$ in Cohort B, 22.2\% in Cohort A, and 18.9\% in SOURCE). This arguably raises questions about the generalizability of the randomized trial data to clinical practice.

4.3.2.3.1. Quality of Life. The quality-of-life results from Cohort B arm, the inoperable cohort, TAVR patients had improvement in the 6-minute walk performance compared with baseline $(P=.002)$, whereas standard therapy patients did not $(P=.67) .{ }^{15}$ In addition, TAVR patients were less symptomatic (New York Heart Association class), had reduced hospitalization stay, and improved physical functioning compared with standard therapy. In the high-risk cohort, both New York Heart Association class and 6-minute walk test favored TAVR at 30 days, but the differences were not significant at 1 year. TAVR patients had shorter index hospitalization length of stay ( 8 vs 12 days, $P<.001$ ). Quality of life as assessed by disease-specific measures (Kansas City Cardiomyopathy Questionnaire [KCCQ]) and by general health-related quality of life (Short Form-12 Health Questionnaire) improved at 1, 6, and 12 months in the TAVR group and were significantly higher than in the control arm $(P<0.001)$. This supports that general and diseasespecific quality of life are improved with TAVR to 1 year over standard care among inoperable patients ${ }^{122}$ (Table 7). The quality of life results from the Cohort A arm of the PARTNER trial were presented in November 2011. The preliminary conclusions were that among patients with severe AS who were at high risk for standard valve replacement, both surgical and transcatheter AVR resulted in substantial improvement in disease-specific and generic health-related quality-of-life assessment over 1-year follow-up, including KCCQ Summary Scale, SF-12 Physical, and SF-12 Mental tests. The benefits were greater at earlier time points in the transfemoral TAVR group and were equivalent at 1 year. ${ }^{123}$

4.3.2.3.2. Continued-Access Protocol. Upon completion of the randomized PARTNER trial, patients have been allowed to have access to TAVR under a continued-access protocol. Enrollment in the randomized continued-access cohort was initiated following completion of the enrollment for PARTNER cohort B trial. From March to September 2009, 91 inoperable patients were enrolled-41 were randomized to TAVR and 50 to standard care. Both short-term (30 days) and long-term (6 months to 1 year) results have been reported (http://www.fda.gov/downloads/Advisory Committees/CommitteesMeetingMaterials/MedicalDevices/ MedicalDevicesAdvisoryCommittee/CirculatorySystem DevicesPanel/UCM262935.pdf). However, betweengroup analyses were not conducted due to the small sample size. Enrollment in nonrandomized continued-access cohort was initiated in September 2009 after both cohorts of 
TABLE 7. Quality of life and symptom assessment in TAVR trials

\begin{tabular}{|c|c|c|c|c|}
\hline Study population & NYHA functional class & 6-Minute walk & Questionnaire & Other \\
\hline $\begin{array}{l}\text { PARTNER B (trial) TAVR } \\
\text { vs placebo (multicenter; } \\
\mathrm{N}=358)^{15,122}\end{array}$ & $\begin{array}{c}\text { More class I, II with TAVR at } \\
1 \text { year }(74.8 \% \text { vs } 42.0 \%)\end{array}$ & $\begin{array}{l}\text { TAVR improved walk time } \\
\text { pre/post at } 1 \text { year; no } \\
\text { change in no-TAVR group }\end{array}$ & $\begin{array}{l}\text { KCCQ; marked improvement } \\
\text { with TAVR at } 1 \text { year; SF12; } \\
\text { improvement in physical } \\
\text { and mental HRQOL with } \\
\text { TAVR }\end{array}$ & $\begin{array}{l}\text { TAVR had fewer } \\
\text { rehospitalizations } \\
\text { at } 1 \text { year }\end{array}$ \\
\hline $\begin{array}{l}\text { PARTNER A (trial) TAVR vs } \\
\text { SAVR (multicenter; } \\
\mathrm{N}=699)^{124}\end{array}$ & $\begin{array}{l}\text { More class I, II with TAVR at } \\
30 \text { days; no difference } \\
\text { between TAVR and SAVR } \\
\text { at } 1 \text { year }\end{array}$ & $\begin{array}{l}\text { TAVR improved walk time at } \\
30 \text { days compared with } \\
\text { SAVR; no difference } \\
\text { between TAVR and SAVR } \\
\text { at } 1 \text { year }\end{array}$ & NR & Shorter LOS with TAVR \\
\hline
\end{tabular}

HRQOL, Health-related quality of life; $K C C Q$, Kansas City Cardiomyopathy Questionnaire; $L O S$, length of stay; $N R$, not reported; $Q O L$, quality of life; SAVR, surgical aortic valve replacement; $T A V R$, transcatheter aortic valve replacement.

PARTNER had completed randomized enrollment. Over 600 patients with transfemoral TAVR are being followed currently in this cohort.

\subsubsection{TAVR-Specific Clinical Issues}

4.3.2.4.1. Stroke. Stroke is one of the major adverse events associated with TAVR. Standardized criteria for the

\section{TABLE 8. Stroke}

\section{Stroke diagnostic criteria}

Rapid onset of a focal or global neurological deficit with at least 1 of the following: change in level of consciousness, hemiplegia, hemiparesis, numbness or sensory loss affecting 1 side of the body, dysphasia or aphasia, hemianopia, amaurosis fugax, or other neurological signs or symptoms consistent with stroke

Duration of a focal or global neurological deficit $\geq 24 \mathrm{~h}$; OR $<24 \mathrm{~h}$, if therapeutic intervention(s) were performed (eg, thrombolytic therapy or intracranial angioplasty); $\mathbf{O R}$ available neuroimaging documents a new hemorrhage or infarct; OR the neurological deficit results in death

No other readily identifiable nonstroke cause for the clinical presentation (eg, brain tumor, trauma, infection, hypoglycemia, peripheral lesion, pharmacological influences)*

Confirmation of the diagnosis by at least 1 of the following:

Neurology or neurosurgical specialist

Neuroimaging procedure (MR or CT scan or cerebral angiography)

Lumbar puncture (ie, spinal fluid analysis diagnostic or intracranial hemorrhage)

\section{Stroke definitions}

Transient ischemic attack:

New focal neurological deficit with rapid symptom resolution (usually 1 to $2 \mathrm{~h}$ ), always within $24 \mathrm{~h}$

Neuroimaging without tissue injury

Stroke: (diagnosis as above, preferably with positive neuroimaging study) Minor-modified Rankin score $<2$ at 30 and 90 days $\dagger$

Major-modified Rankin score $\geq 2$ at 30 and 90 days

Reprinted with permission from Leon et al. ${ }^{96} C T$, Computed tomography; $M R$, magnetic resonance. *Patients with nonfocal global encephalopathy will not be reported as a stroke without unequivocal evidence based upon neuroimaging studies. $\dagger$ Modified Rankin score assessments should be made by qualified individuals according to a certification process. If there is discordance between the 30- and 90-day Modified Rankin scores, a final determination of major versus minor stroke will be adjudicated by the neurology members of the clinical events committee. definition of stroke endpoints for TAVR clinical trials have been published by the VARC (Table 8). The incidence of stroke depends on the assessment technique used for ascertainment. In the PARTNER Cohort A, the risk of clinically apparent "major" stroke defined as modified Rankin score $\geq 2$ was $3.8 \%$ at 30 days and $5.1 \%$ at 1 year among the TAVR group compared with $2.1 \%$ and $2.4 \%$, respectively, in the surgical group. ${ }^{124}$ In the PARTNER Cohort $\mathrm{B}$, the stroke risk was $5 \%$ with TAVR compared with $1.1 \%$ with standard therapy at 30 days and $8.4 \%$ versus $3.9 \%$ at 1 year. ${ }^{15}$ Using magnetic resonance imagingdiffusion weighted imaging (MRI-DWI) studies, the incidence of cerebral ischemic lesions post-TAVR has been reported to be as high as $68 \%$ to $84 \%$ in some studies, although clinically apparent stroke was reported in $>4 \%$ of cases. ${ }^{125-128}$ Thus, the clinical significance of these new CMR-defined lesions post-TAVR is not clear.

Most stroke cases are due to thromboembolism from the valve site or due to atherothrombotic emboli originating from ulcerative plaque in the great vessels such as the aortic arch. Such particles can be dislodged during catheter manipulation and embolize to the carotids or vertebrals to cause occlusions of distal intracerebral branch arteries. Other potential causes include hypotension associated with rapid ventricular pacing or hemodynamic instability during the procedure, and rarely due to aortic dissection complicating TAVR. It is important to recognize that many patients who have AS may also have other causes for an ischemic stroke such as age, hypertension, diabetes, or other cardiac conditions, including atrial fibrillation, which is a potent risk factor for cardioembolic stroke. ${ }^{69}$ Differentiating the cause of the stroke is not always easy, but most trials and registries define strokes within 30 days of an interventional procedure as attributable to the procedure. After 30 days, other comorbid risk factors may account for stroke, which might, therefore, not be attributable to the prosthetic valve. Diagnostic evaluations are needed to assess the neck and cerebral vessels, cardiac function, and other potential causes of stroke in order to differentiate the stroke subtype and embark on the best treatment to 
prevent a recurrent stroke. ${ }^{129,130}$ Nearly two thirds of the strokes related to TAVR at 1 year occurred within the first 30 days in PARTNER Cohort B (13/20), suggesting that most events were likely procedure-related. ${ }^{15}$ The incidence of stroke may lessen as patient selection becomes more refined, delivery systems improve in their profile, and embolic protection devices and protocol-driven antithrombotic regimens are routinely used during TAVR.

4.3.2.4.2. Conduction Defects. Atrioventricular conduction disturbances after TAVR are associated with many patientrelated and procedural-related factors, including preoperative comorbid status, the degree and bulkiness of aortic valve and annular calcification, interventricular septal thickness, pre-existing electrocardiogram abnormalities, the depth of prosthesis implantation, and the profile of the implanted prosthesis. ${ }^{131,132}$ Unlike conventional AVR, where there may be localized trauma due to decalcification of the annulus and/or suture placement in the proximity of the $\mathrm{AV}$ node or the bundles, TAVR may cause conduction abnormalities through mechanical impingement of the conduction system by the prosthesis.

The incidence of new left bundle-branch block and complete heart block after TAVR ranges from $14 \%$ to $83 \%$ and $19 \%$ to $22 \%$, respectively. Patients with pre-existing right bundle-branch block may be at the highest risk for the development of complete heart block and the need for subsequent pacing. ${ }^{133}$ The majority of conduction abnormalities occur prior to actual valve implantation, with $46 \%$ occurring during balloon aortic valvuloplasty, $25 \%$ during balloon/prosthesis positioning and wire-crossing of the aortic valve, and the remaining $29 \%$ during prosthesis expansion. ${ }^{134}$ The incidence of complete heart block requiring permanent pacemaker implantation has been higher with the CoreValve (19.2\% to $42.5 \%$ ) than with the Sapien valve $(1.8 \%$ to $8.5 \%)$, potentially due to its larger profile and extension low into the LV outflow tract. In the most recent UK Registry, pacemakers were implanted in $24.4 \%$ of patients receiving the CoreValve.

Overall, permanent pacemaker implantation rates with the CoreValve, but not Sapien valve, are higher than conventional surgical AVR rates of $1 \%$ to $10 \%$. The need for permanent pacemaker implantation occurs early postprocedure and rarely after hospital discharge. The need for permanent pacemaker implantation has no effect on survival, both early at 30 days postprocedure and late at 1 year. ${ }^{135}$ Continuous postoperative electrocardiogram monitoring should be performed in all patients early after TAVR procedures. Patients with pre-existing or new conduction abnormalities and those receiving the CoreValve device may require longer monitoring.

4.3.2.4.3. Vascular Complications. Vascular complications are the most frequent adverse outcome of TAVR and are especially common with transfemoral approach. ${ }^{136}$ These complications relate to the large-caliber sheaths necessary for device deployment, as well as severe atherosclerosis of the arteries, which is common. ${ }^{137}$ Center/operator experience, the degree and location of vascular calcification, vascular tortuosity, and sheath-to artery ratio are predictors of major vascular complication. ${ }^{137,138}$ Major vascular complications are classified in accordance with the definitions provided by the VARC and include aortic dissection, perforation, rupture, or bleeding requiring significant blood transfusions, or additional percutaneous or surgical intervention. ${ }^{96}$ Incidence of major vascular complications ranges from $2 \%$ to $26 \%$ with transfemoral access and is related to vessel size, tortuosity, and degree of aortoiliac occlusive disease and from $5 \%$ to $7 \%$ with transapical access. ${ }^{107,109,111,116,139}$

Subclavian access may represent an alternative approach in some patients in whom transfemoral or transapical direct aortic access cannot be utilized. Subclavian artery injury is rare with such access although transient brachial plexus neuropathy has been reported with this approach. ${ }^{140}$ As delivery systems improve in their profile, the incidence of these complications will lessen. ${ }^{136,141}$ Of note, left subclavian arterial access for TAVR may not be appropriate in patients with prior CABG with left internal thoracic arterial graft because temporary interruption of blood flow in the left internal thoracic artery may cause coronary insufficiency.

4.3.2.4.4. Patient Preferences. Informed consent requires the patient and/or support system be appropriately informed of the procedure benefits and risks, possess personal decision-making capacity, and ultimately be able to make a voluntary decision. Older adults often rely on trusted physicians, family, or friends to participate and guide medical decision making at the point of medical care. A central goal in this interaction is the exchange of relevant, detailed information about treatment strategies delivered in terminology that is understood by the patient and family. This patient-centric educational effort is essential in providing the patient and family information to facilitate interaction with the health care team, and promote personalized decision making for each patient. It is important to remember that risk tolerance and patient expectations vary across many patient populations. Thus, a thorough review of personalized risk/benefit profile is essential for each patient undergoing an invasive procedure.

4.3.2.4.5. Benefit/Risk Assessment. The complex task of balancing the benefit and risk of TAVR depends upon accurate information regarding prognosis for survival, morbidity, and expected quality of life. Ideally, an accurate validated model that predicts both in-hospital and longterm outcome should guide this analysis, help educate 

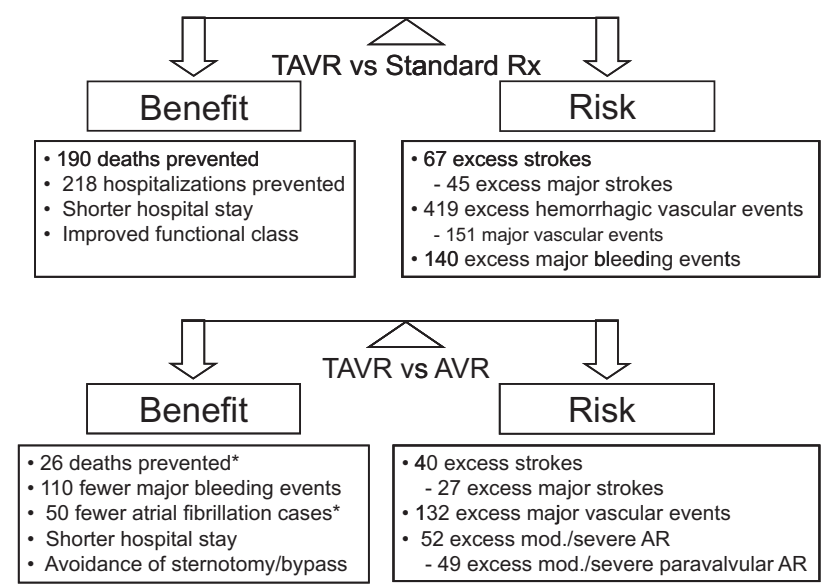

FIGURE 4. Benefit/risk balance in the PARTNER trial. Data are shown for every 1000 patients treated with TAVR instead of standard treatment in Cohort B (above) or standard AVR in Cohort A (below). The excesses listed are not mutually exclusive, because some patients had more than 1 event. Only data with statistically significant differences at 1 year of follow-up are shown except for deaths* $(P=.44)$ and atrial fibrillation $\dagger$ $(P=.07)$ in Cohort A. $A R$, Aortic valvular regurgitation; $A V R$, surgical aortic valve replacement; TAVR, transcatheter aortic valve replacement.

patients and their families, and effectively manage safety tradeoffs and health care expenditure. Such a model would include some assessment of the relative role of severe AS versus comorbidity (eg, COPD in the etiology of symptoms such as dyspnea). Although several risk models have been developed for prognostication after cardiac surgery, they are limited by modest performance with regards to discriminatory ability, calibration, and face validity. It is not clear whether these models for conventional cardiac surgery are similarly predictive of outcome of patients being considered for TAVR. An additional important issue relates to the lack of a formal assessment of other aspects of treatment risk and benefit (eg, gait, cognition, frailty) in these risk models. Thus, better performing risk models are needed that include a wide spectrum of prognostic variables using contemporary data in relevant populations for a TAVR-specific risk algorithm.

In TAVR candidates, the benefits of avoidance of sternotomy and cardiopulmonary bypass with its attendant complications and prolonged recovery/hospitalization by applying TAVR appear to come at the price of potentially serious vascular and technical complications and increased hazards of stroke and paravalvular AR (Figure 4). For prohibitively high-risk inoperable patients, such a tradeoff is acceptable given the documented statistically-significant and clinically-important mortality benefit and functional improvement. For high surgical risk patients in whom mortality benefit has not been proven, the findings present a dilemma, given that the irreversible effects of stroke might be of greater potential clinical significance in terms of long-term disability, permanent dependency, and increased societal costs than the complications of sternotomy and bleeding. Although bleeding occurs acutely, is often overt, and has immediate clinical impact leading to increased length of stay and resource utilization, a "causal link" to adverse long-term clinical outcome remains unproven. Ultimately, the relative weights both patients and physicians assign to the utility associated with these periprocedural hazards is likely to impact individual case-based benefit/risk assessment and decision making. Cost considerations are also likely to materially impact the adoption of TAVR in treatment algorithms for AS.

\subsubsection{Medtronic CoreValve U.S. Pivotal Trial}

The U.S. pivotal trial with the Medtronic CoreValve selfexpanding valve is currently enrolling patients at 40 sites (NCT \# 01240902). Patients are allocated into either an Extreme Risk cohort, similar to Cohort B inoperable patients in the PARTNER trial or a High Risk cohort analogous to PARTNER Cohort A. There will be 487 patients enrolled in the extreme-risk group with an additional 100 patients with inadequate iliofemoral access placed in a nested registry of alternative access with either subclavian or direct aortic approaches. The High Risk cohort will enroll 790 patients in a 1:1 randomization between TAVR and surgical AVR.

\section{INTEGRATION OF TAVR INTO CLINICAL PRACTICE}

\subsection{Patient Evaluation and Management}

\subsubsection{Multidisciplinary Team}

The creation of a multidisciplinary team that includes the patient in the decision process in choosing the most appropriate form of treatment for AS including AVR (ie, surgical or percutaneous) is essential. It is similar in concept to the "heart team" approach for CAD. ${ }^{142}$ Factors such as sex, race, availability, experience, and institutional commitment to managing very high-risk patients, technical skills, local results, referral patterns, and patient preference all may have an impact on the decision-making process and should be taken into account by this multidisciplinary team. Ideally, such a team would be comprised of the patient's primary cardiologist, cardiac surgeon, interventional cardiologist, echocardiographer, imaging specialists-CT or CMR, heart failure and valve disease specialist, cardiac anesthesiologist, nurse practitioner, and cardiac rehabilitation specialists. Such a strategy would result in input from multiple skill sets with the goal being the best possible course of therapy leading to the best possible clinical outcome for the specific patient.

Localization of a heart team working together in a valve clinic will help optimize the functions of the valve team. Such a clinic should combine clinical cardiac care, 
advanced imaging capability, and surgical consultation to provide centralized assessment and treatment options for complex valve disorders. Patients referred to a valve clinic should be assessed by a cardiologist and a cardiovascular surgeon to discuss the options for surgical intervention if indicated. Prior diagnostic studies should be reviewed and additional diagnostic imaging (echocardiography, TEE, MDCT [multidetector computed tomography], CMR) performed as clinically indicated. Overall, a valve clinic should offer patients a personalized approach for the evaluation and treatment of complex valve disorders with the availability of a cardiologist and a cardiac surgeon specializing in valve disorders.

\subsubsection{Patient Selection}

\subsubsection{Inclusion/Exclusion Criteria}

TAVR is appropriate currently only for a highly select population and the valve team should systematically identify the characteristics that define that population with most benefit and acceptable risk. These identification criteria should be operationalized into practice and may evolve over time with this new technology as new data become available.

The inclusion and exclusion criteria in extant randomized studies are generally appropriate for use in clinical practice (Table 9). These vary somewhat, but there are some criteria common to most studies. Some criteria can be precisely identified with objective measurements, but many require subjective estimates based on clinical judgment. These subjective assessments are at least as important as the objective determinations and necessarily create some variability in the process of patient selection. The criteria presented here are based on current technology and experience. As technology improves and experience is gained, it is likely that many of these criteria will change to expand TAVR to different populations that will be optimally treated with the next generation of devices. In addition, the arbitrary criteria such as qualifying aortic AVA measurement within 45 days within the procedure will be modified and made more flexible.

\subsubsection{Specific Patient Subsets}

5.1.2.2.1. Porcelain Aorta, Friable Aortic Atheroma, Radiation Heart Disease. Occasionally, otherwise fairly healthy candidates for AVR will have local factors such as prior radiation therapy to their mediastinum and/or severe calcific changes within their ascending aorta ("porcelain aorta") that add significant risk to a traditional open AVR. Rarely, transesophageal echocardiography will reveal advanced atherosclerosis with mobile and pedunculated atheromata that also increase risk for stroke or a major embolic event with traditional TAVR. Cases such as these are approached individually and the correct approach is at best an educated judgment on the part of the surgical team. TAVR offers an alternative for the treatment of AS when there is severe circumferential calcification (porcelain aorta) or heavy atherosclerotic disease burden in the ascending aorta. ${ }^{143}$ Patients with extensive atherothrombotic burden involving the ascending aorta should be approached very carefully irrespective of whether either a transapical or transfemoral procedure because of the potential for embolization.

5.1.2.2.2. Very Elderly. Advanced age has important implications, as typically these patients have several comorbid conditions (in addition to advanced age) that increase the risk of AVR or TAVR. Functional status and comprehensive assessment of comorbidities including CAD, history of transient ischemic attack or stroke, chronic kidney disease, and dementia should be performed. Finally, risk and benefit, including prognosis of existing conditions, should be thoroughly discussed with the patient and family as part of the initial meeting with the TAVR team and should include a review of postprocedural complications that may extend hospitalization. On the other hand, successful procedures result in improvement in dyspnea, a heightened energy level, and an overall improved quality of life. Life expectancy can be prolonged, since the mortality of medicallytreated symptomatic severe AS carries a high mortality.

As noted above, symptoms usually improve following valve replacement, but a caveat exists for elderly patients regarding dyspnea and the presence of $\mathrm{LVH}$. LVH is seen in $54 \%$ of men and $81 \%$ of women with severe $\mathrm{AS}^{144-147}$ whereas men more often have less LVH, some LV chamber enlargement, and some reduction in EF. Occasionally women will have such severe diastolic dysfunction that even when the afterload stress is relieved by TAVR, elevated LV filling pressures may result in persistent symptoms of shortness of breath. Since LVH may eventually regress following TAVR, shortness of breath may also eventually improve over several months following valve replacement. Men, who tend to have a greater degree of LV myocardial fibrosis and abnormal LV collagen network patterns, ${ }^{148}$ may have more inherent reduced contractility, so that relieving afterload with TAVR may also not result in early or marked symptomatic improvement. When discussing TAVR with the very elderly, they should be made aware that symptomatic improvement may be delayed or minimal in some cases.

5.1.2.2.3. Frailty and Futility Versus Utility. As previously discussed, the concepts of frailty and futility will assume central importance in patient selection for TAVR by virtue of the extensive comorbidities present in this population. Frailty is an important and frequent condition in elderly patients and should be considered when dealing with invasive 
TABLE 9. Patient selection: Inclusion and exclusion criteria in clinical trials

Inclusion criteria

1. Patient has calcific aortic valve stenosis with echocardiographically derived criteria: mean gradient $>40 \mathrm{~mm} \mathrm{Hg}$ or jet velocity $>4.0 \mathrm{~m} / \mathrm{s}$ and an initial AVA of $<0.8 \mathrm{~cm}^{2}$ or indexed EOA $<0.5 \mathrm{~cm}^{2} / \mathrm{m}^{2}$. Qualifying AVA baseline measurement must be within 45 days of the date of the procedure.

2. A cardiac interventionalist and 2 experienced cardiothoracic surgeons agree that medical factors either preclude operation or are high risk for surgical AVR, based on a conclusion that the probability of death or serious, irreversible morbidity exceeds the probability of meaningful improvement. The surgeons' consult notes shall specify the medical or anatomic factors leading to that conclusion and include a printout of the calculation of the STS score to additionally identify the risks in the patient. At least 1 of the cardiac surgeon assessors must have physically evaluated the patient.

3. Patient is deemed to be symptomatic from his/her aortic valve stenosis, as differentiated from symptoms related to comorbid conditions, and as demonstrated by NYHA functional class II or greater.

Exclusion criteria (candidates will be excluded if any of the following conditions are present)

1. Evidence of an acute myocardial infarction $\leq 1$ month (30 days) before the intended treatment (defined as: Q-wave MI, or non-Q-wave MI with total $\mathrm{CK}$ elevation of $\mathrm{CK}-\mathrm{MB} \geq$ twice normal in the presence of MB elevation and/or troponin level elevation [WHO definition])

2. Aortic valve is a congenital unicuspid or congenital bicuspid valve, or is noncalcified

3. Mixed aortic valve disease (aortic stenosis and aortic regurgitation with predominant aortic regurgitation $>3+$ )

4. Hemodynamic or respiratory instability requiring inotropic support, mechanical ventilation, or mechanical heart assistance within 30 days of screening evaluation

5. Need for emergency surgery for any reason

6. Hypertrophic cardiomyopathy with or without obstruction

7. Severe left ventricular dysfunction with $\mathrm{LVEF}<20 \%$

8. Severe pulmonary hypertension and RV dysfunction

9. Echocardiographic evidence of intracardiac mass, thrombus or vegetation

10. A known contraindication or hypersensitivity to all anticoagulation regimens, or inability to be anticoagulated for the study procedure

11. Native aortic annulus size $<18 \mathrm{~mm}$ or $>25 \mathrm{~mm}$ as measured by echocardiogram*

12. MRI confirmed CVA or TIA within 6 months (180 days) of the procedure

13. Renal insufficiency (creatinine $>3.0 \mathrm{mg} / \mathrm{dL}$ ) and/or end-stage renal disease requiring chronic dialysis at the time of screening

14. Estimated life expectancy $<12$ months ( 365 days) due to noncardiac comorbid conditions

15. Severe incapacitating dementia

16. Significant aortic disease, including abdominal aortic or thoracic aneurysm defined as maximal luminal diameter $5 \mathrm{~cm}$ or greater; marked tortuosity (hyperacute bend), aortic arch atheroma (especially if thick [ $>5 \mathrm{~mm}$ ], protruding or ulcerated) or narrowing (especially with calcification and surface irregularities) of the abdominal or thoracic aorta, severe "unfolding" and tortuosity of the thoracic aorta

17. Severe mitral regurgitation

$\overline{A V A}$, Aortic valve area; $A V R$, aortic valve replacement; $C K$, creatine kinase; $C V A$, cerebrovascular accident; $E O A$, effective orifice area; $L V E F$, left ventricular ejection fraction; $M B$, MB isoenzyme; $M I$, myocardial infarction; $M R I$, magnetic resonance imaging; $N Y H A$, New York Heart Association; $R V$, right ventricular; STS, Society of Thoracic Surgeons; $T I A$, transient ischemic attack; $W H O$, World Health Organization. *The boundaries of annulus size will continue to change in concert with changing device size.

care in older adults. ${ }^{149}$ Although it can have significant overlap with disability and comorbidity, it is a distinct syndrome and is characterized by a vicious cycle of decreasing muscle mass, energy expenditure, and malnutrition culminating in vulnerability to adverse events. ${ }^{150}$ In the PARTNER trial, frailty was present in as many as $23 \%$ of patients in Cohort B and $16 \%$ in Cohort A. Besides comorbidities, and frequently in combination with them, it is likely to play a role in the assessment of the individual's candidacy for invasive care and therefore in withholding any intervention in nearly one half of high-risk patients with AS. ${ }^{151}$ It is important to consider that frailty may be a reversible physiological phenotype in some cases, and therefore it is premature to consider this a permanent characteristic of the individual patient. To the extent that AS may contribute to the declining health state, AVR or TAVR may reverse frailty. In this case, frailty may be a marker for treatment benefit. Conversely, if the individual is frail from multiple other organ system declines, frailty may be a marker of treatment risk.
The impact of frailty on the clinical course and outcome of patients presenting with severe AS is beginning to be investigated but is difficult to assess because of its multidimensional phenotype and the lack of a clear and agreedupon assessment. The definition of frailty used in recent studies ranges from the qualitative "eyeball test" to more quantitative scores such as the Fried Frailty Index. ${ }^{150} \mathrm{~A}$ simple test for defining frailty is a timed gait speed over 5 $\mathrm{m}$. In a recent Canadian study, ${ }^{152}$ a time of $>6$ seconds as a measure of frailty was found to be an independent predictor of mortality compared with the STS risk algorithm alone. As such, it has recently been added to the STS database upgrade (Version 2.73, July 1, 2011) and will be uniformly collected in patients undergoing cardiac surgery. ${ }^{152}$ Future studies should aim at developing more reliable and reproducible ways of identifying frailty, as well as incorporating these assessments in development of risk and benefit prediction.

Futility is also an important consideration for TAVR. There may be some patients in whom this procedure 
should not be performed because the clinical condition is too far advanced; in these patients, even a successful technical procedure is futile and does not improve health outcomes.

Therapeutic futility may be determined based upon: (1) lack of medical efficacy, as judged by the patient's physician; or (2) lack of a meaningful survival, as judged by the personal values of the patient. ${ }^{153,154}$ Although therapeutic futility may be invoked to justify denial, limitation, or withdrawal of care, the threshold for defining it is unclear, controversial, and often viewed differently by different stakeholders. In the PARTNER trial, the criterion for inoperability-used as a surrogate for futility with regards to surgical intervention-was an estimate of probability of death or serious, irreversible morbidity $\geq 50 \%$ by a cardiologist and 2 experienced cardiothoracic surgeons. ${ }^{15}$ Despite successful correction of AS leading to an absolute $20 \%$ survival advantage, there was still $30 \%$ mortality in the TAVR treatment arm at 1 year, mainly due to noncardiac causes. The key to treatment in this group of "inoperable" patients is to define the "futility versus utility" treatment paradigm. Clearer definition of comorbid conditions that adversely affect survival despite successful valve implementation as well as quality of life and health economic assessment in those "inoperable" patients is crucial so that this therapy is appropriately used in patients likely to benefit (utility) compared with those unlikely to benefit despite successful therapy (futility). Although some might argue that it is inappropriate and misleading to say that treatment is futile simply because the probability that it will succeed is small, especially given the substantial uncertainty in our ability to prognosticate in individual patients and lack of validated tools that universally discriminate survivors from nonsurvivors of critical illness, it is nonetheless important to define meaningful cutoff points. This is particularly true when, in the course of a progressive illness, continued use of resources other than measures for comfort, is no longer reasonable, practical, or appropriate. Ultimately, these decisions must be guided by what our society considers to be the inherent value of human life and the resultant financial burden society is willing to bear for the provision of modern public health care. ${ }^{155}$

\subsubsection{Care Plan in Candidates for TAVR}

The health care team needs to be intimately involved in discussions on risk/benefits including detailed information on individualized risks for each patient and alignment of quality-of-life expectations. Failure to understand and comply with a plan of care may account for dissatisfaction with procedural outcomes and potential rehospitalizations. ${ }^{156,157}$ One critical intervention to ensure effective care coordination and transition is that of comprehensive plan of care and educational material given to patient and/or caregivers prior to the planned procedures, and again during and after hospitalization. This process may encourage full participation of the patient and family about adherence to medication therapy and activity recommendations. Transitions of care and follow-up will be improved by discussion and written instructions reviewed with each patient including medications, timely follow-up with the various health care professionals involved with the patient's ongoing care, and appropriate postprocedural activities. The ongoing care and coordination with the cardiovascular care team may decrease likelihood of readmission and improve overall adherence. Health care providers should pay close attention to psychosocial and socioeconomic issues that the patient and family face, including access to care, risk of depression, and health care disparities. ${ }^{158-160}$

\subsubsection{Imaging Assessment}

Imaging plays an essential role in patient selection and procedural planning, performance, and follow-up. ${ }^{161}$ In each of these steps, optimal imaging can help to enhance successful outcome. There is variability in the specific imaging protocols preferred in individual institutions. This variability is the result of institutional and individual experience and equipment, as well as the specific patient characteristics to be considered.

\subsubsection{Echocardiography}

The following general recommendations can be made for echo assessment of patients being considered for TAVR. More detailed instructions can be found in a recent expert consensus statement from the American Society of Echocardiography and the European Society of Echocardiography. ${ }^{161}$

5.1.4.1.1. Annulus Size and Cusp and Root Anatomy. Accurate assessment of annular size is critical. Underestimation of annular size could lead to selection and deployment of a valve which is too small, with risks of poor hemodynamics, paravalvular regurgitation, and valve migration and embolism. Overestimation of annular size and placement of a valve that is too large can lead to other adverse outcomes, including incomplete deployment (with both valvular and paravalvular regurgitation) or catastrophic annular rupture. In general, all TAVRs are designed to be deployed in annuli that are slightly smaller than the prosthesis size. This oversizing is required because the valves are sutureless and depend on radial force to prevent dislodgement. For the initial Sapien valves, the 23$\mathrm{mm}$ valve was designed for $18-\mathrm{mm}$ to $22-\mathrm{mm}$ annuli, whereas the 26-mm prosthesis was designed for 21-mm to 25-mm annuli. The Sapien XT valve, with 23-mm, 26-mm, and $29-\mathrm{mm}$ sizes, is designed for annuli from $18 \mathrm{~mm}$ to 27 $\mathrm{mm}$. The CoreValve has $26-\mathrm{mm}, 29-\mathrm{mm}$, and $31-\mathrm{mm}$ prosthesis sizes (using a different sizing convention from the 
Sapien valve) designed for annuli from $20 \mathrm{~mm}$ to $23 \mathrm{~mm}$ for the $26-\mathrm{mm}$ prosthesis, $24 \mathrm{~mm}$ to $27 \mathrm{~mm}$ for the $29-\mathrm{mm}$ prosthesis, and $26 \mathrm{~mm}$ to $29 \mathrm{~mm}$ for the 31-mm annuli. Annular dimensions can be measured with either TTE or TEE. ${ }^{162}$ With either modality, the annular anteroposterior diameter is measured from a long-axis view. Care must be taken to identify the true annulus, not overlying calcium. Measurements are made in systole at the hinge point of the leaflets into the LVOT with a trailing edge to leading edge convention. Because the annulus is often elliptical, optimal assessment should include measurement of the transverse (coronal) diameter, using the short-axis view, ideally with biplane TEE approach or CT, which allows simultaneous long- and shortaxis interrogation of the annular plane.

\subsection{Aortic Root Disease and Ascending Aortic Di-} mensions. Assessment of cusp and root anatomy is also critical. The PARTNER trial excluded all patients with bicuspid aortic valves for concern that such valves might distort the prosthesis, leading to paravalvular regurgitation. Thus, TAVR in any nontricuspid valve would be considered an off-label use, though successful treatment of bicuspid valves has been reported. ${ }^{163}$ It is often difficult to determine cusp anatomy in the densely calcified valves commonly treated by TAVR. In this setting, CT or review of old echocardiograms may allow better assessment of the underlying anatomy. Pathology reviews have demonstrated progressive increase with age in the proportion of trileaflet valves in severe AS patients, from $15 \%$ in those under 60 years to $60 \%$ over 70 years $\left(72 \%\right.$ for those over 80 years). ${ }^{2}$ Of note, this study showed that even pathological examination cannot determine cusp anatomy in some heavily distorted valves.

Several issues must be considered in assessing root anatomy and pathology. Care must be taken to assure that valve deployment will not compromise the coronary ostia, either from the device itself or from cusp calcification being shifted and displaced into the coronary. In general, CT scanning provides a more comprehensive assessment of the relationship of the coronary arteries to the annulus and valve leaflets, demonstrating an average annular-left coronary artery distance of $13.4 \pm 3.2 \mathrm{~mm}$ and annular-right coronary artery distance of $13.6 \pm 2.8 \mathrm{~mm} .{ }^{164}$ Nevertheless, echo, particularly TEE, can measure the distance from the aortic valve annulus to the right coronary ostium. Since the left coronary does not lie in a standard TEE or TTE imaging plane that intersects the annulus, measurement from 3D datasets may be a feasible approach for this.

Accurate assessment of the aortic root and tubular portion is also important. The CoreValve Revalving System is designed with a supra-annular location of the porcine pericardial valve, located in the sinus of Valsalva. As a result, the CoreValve nitinol frame has a longer length than conventional surgical valves, ranging from $52 \mathrm{~mm}$ (for the $31-\mathrm{mm}$ valve) to $55 \mathrm{~mm}$ (for the $26-\mathrm{mm}$ valve) including its deployment hooks. It is recommended that the upper dimensions of the tubular aorta measured at $45 \mathrm{~mm}$ above the annulus be $40 \mathrm{~mm}$ for the $26-\mathrm{mm}$ valve and $43 \mathrm{~mm}$ for the 29-mm and 31-mm CoreValve prostheses.

Preprocedural assessment of AR in TAVR candidates should be governed by guidelines from the American Society of Echocardiography. ${ }^{165}$ This assessment is based on multiple parameters, including LV size, AR jet size and morphology, AR pressure half-time, and diastolic flow reversal in the aortic arch. Patients with $>3+$ AR were excluded from the PARTNER trial and should be considered relatively contraindicated for TAVR.

\subsection{Three-Dimensional Echocardiography. Real-} time 3D TEE is an important modality for preprocedural and intraprocedural assessment of TAVR patients. ${ }^{166,167}$ Similar to MDCT and CMR, it can help with precise assessment of the aortic root and annulus, potentially helping reduce the chance for prosthesis-sizing error in patients. However, multiple studies have demonstrated significant differences in dimensions of the aortic root and annulus measured by 2D TTE, 2D TEE, 3D TEE, and MDCT. ${ }^{166,167}$ Hence, it is imperative to realize that the imaging technique utilized might impact TAVR size selection and strategy. TEE, including real-time 3D TEE can help evaluate the extent of and precisely locate the jet of AR following prosthesis implantation.

\subsubsection{Tomographic Imaging}

5.1.4.2.1. Rationale for Tomographic Imaging. Optimizing outcome relies heavily on image guidance for patient selection, preprocedural planning, and intraoperative decision making. ${ }^{168}$ Correct positioning of the prosthesis relative to the annulus is critical. If valve deployment is too high, increased risk of paravalvular regurgitation, aortic injury, coronary occlusion, or embolization of the prosthesis can occur. If positioning is too low, mitral valve dysfunction, heart block, paravalvular regurgitation, or embolization into the left ventricular cavity can occur. ${ }^{169}$ In addition, the relatively large delivery catheters currently required for valve insertion are associated with the risk of vascular complications, necessitating assessment of iliofemoral vasculature. This has led to the application of 3D imaging approaches for TAVR, including CT, CMR, 3D echocardiography, and C-arm $\mathrm{CT}^{162,166,170,171}$ (Table 10).

\subsection{Multidetector Computed Tomography. MDCT} provides comprehensive assessment of the aortic root, atherosclerotic burden, and course of the thoracoabdominal aorta and its iliofemoral branches (Figure 5). MDCT in the context of TAVR eligibility assessment has become routine in many large-volume centers. ${ }^{172}$ 
TABLE 10. Potential approaches for imaging in TAVR

\section{Preprocedural assessment}

1. Assessment of aortic annular size and shape (CT, CMR, 2D and 3D echocardiography)

2. Assessment of aortic valve for number of cusps, degree of calcification and valve area by planimetry (CT, CMR, 2D and 3D echocardiography)

3. Measurement of the distance between annulus and coronary ostia (CT, CMR, 2D and 3D echocardiography)

4. Planning for precise coaxial alignment of the stent-valve along the centerline of the aortic valve and aortic root (CT)

5. Assessment of aortic dimensions (2D and $3 \mathrm{D}$ echocardiography, CT or CMR) and atherosclerosis (echocardiography, CT, or CMR)

6. Assessment of dimensions and atherosclerosis of iliofemoral vessels (CT, MR, angiography)

Postprocedural assessment

1. Assessment of degree of aortic regurgitation (echocardiography or CMR)

2. Assessment of cerebral embolization (cerebral MRI)

2D, 2-dimensional; 3D, 3-dimensional; $C M R$, cardiac magnetic resonance; $C T$, computed tomography; MRI, magnetic resonance imaging; TAVR, transcatheter aortic valve replacement.

MDCT systems with at least 64 detectors and a spatial resolution of $0.5 \mathrm{~mm}$ to $0.6 \mathrm{~mm}$ are recommended. The specific scan protocols used for assessment vary but generally include imaging of the aortic root and the thoracoabdominal aorta and its iliofemoral branches. ECG-synchronized imaging of the aortic root is important to avoid image quality degradation due to motion artifacts, and image reconstruction is performed at the desired phase of the cardiac cycle (eg, a systolic $30 \%$ to $40 \%$ phase for valve area and annular assessment). Using the retrospectively ECG-gated helical acquisition, CT data can be acquired throughout the entire cardiac cycle, enabling 4D image reconstructions for evaluation of valvular function, albeit at the expense of a higher radiation dose. ${ }^{173}$ Alternatively, prospectively ECG-triggered axial CT data acquisition requires much less radiation; however, images are acquired during a prespecified phase of the cardiac cycle and reconstruction in other phases or $4 \mathrm{D}$ cine loops may not be reconstructible. ${ }^{174}$ However, protocols with newer generation scanners allow prospective acquisition at a lower radiation dose with subsequent display of cine loops. ${ }^{175}$ Although radiation exposure is important to consider with any CT acquisition, it is less a concern in the elderly patients currently considered for TAVR.

Because a standard bolus of $80 \mathrm{~mL}$ to $120 \mathrm{~mL}$ of lowosmolar iodinated contrast is necessary, the benefits versus risks of iodinated contrast need to be carefully weighed. ${ }^{176}$ An alternative approach involves a pelvic scan after intraarterial contrast injection into the infrarenal abdominal aorta (catheter left in place after cardiac catheterization) using a very low dose $(15 \mathrm{~mL})$ of contrast. ${ }^{177}$ If contrast administration is not feasible, a noncontrast scan, although not optimal, still allows the assessment of overall vessel size, calcification, and tortuosity.

As previously mentioned, analysis and measurement of the annulus size and shape are crucial for procedural success. Typical annulus measurements, obtained using 2D TTE or TEE provide a single diameter measurement, assuming a circular annular orifice. ${ }^{178}$ In contrast, 3D CT systolic reconstruction of the annulus orthogonal to the center-axis of the LVOT allows for the assessment of minimal and maximal diameter, circumference, and area measurements. ${ }^{162,164,166,179-183}$ Indeed, these studies have demonstrated that the LVOT is often oval, rather than circular. Hence, multimodality imaging might improve the accuracy of AV measurements and reduce the chance for prosthesis-sizing errors in patients considered for TAVR.

Complete coronary assessment with MDCT is obviously limited in the current population evaluated for TAVR because of the very high prevalence of advanced calcified disease, precluding precise assessment of luminal stenosis. However, MDCT allows measurement of the distance between annulus and coronary ostia, which identifies patients at risk for coronary occlusion during TAVR. Although no definite criteria exist to exclude patients, a $<10 \mathrm{~mm}$ distance might identify increased risk of coronary ostial occlusion. ${ }^{184}$ In this setting, placement of a guidewire or balloon catheter in the left main artery could be considered to ensure access in case of complications.

Although echocardiography is used extensively to assess the aortic valve, cine MDCT can provide incremental value in its assessment, including number of cusps, especially in cases of heavy calcification, where echocardiography can be difficult. Also, cine MDCT can be used to perform planimetry of the aortic valve. ${ }^{180,185}$

Optimal coaxial alignment of the stent valve along the centerline of the aortic valve and aortic root is important during positioning. Ascertainment of the right height to avoid too high or too low placement is important to avoid AR and optimize valve function. ${ }^{169}$ Although traditional assessment of root orientation is performed using multiple invasive aortograms in 1 or 2 orthogonal planes, double-oblique multiplanar MDCT reconstruction allows preprocedural prediction of the aortic root angle. ${ }^{186,187}$ This potentially decreases the number of aortograms required during the procedure, therefore shortening both procedure time and contrast usage, and improves precision of deployment. The emergence of $\mathrm{C}$-arm CT would further allow the incorporation of fusion imaging in the catheterization laboratory. ${ }^{170}$

\subsection{Cardiac Magnetic Resonance. Similar to MDCT,} CMR can also potentially provide comprehensive assessment of the aortic valve, annulus, aortic root, course of the thoracoabdominal aorta and luminal caliber of the iliofemoral branches, without the ionizing radiation. 2D ECGgated noncontrast cine CMR sequences across the aortic 

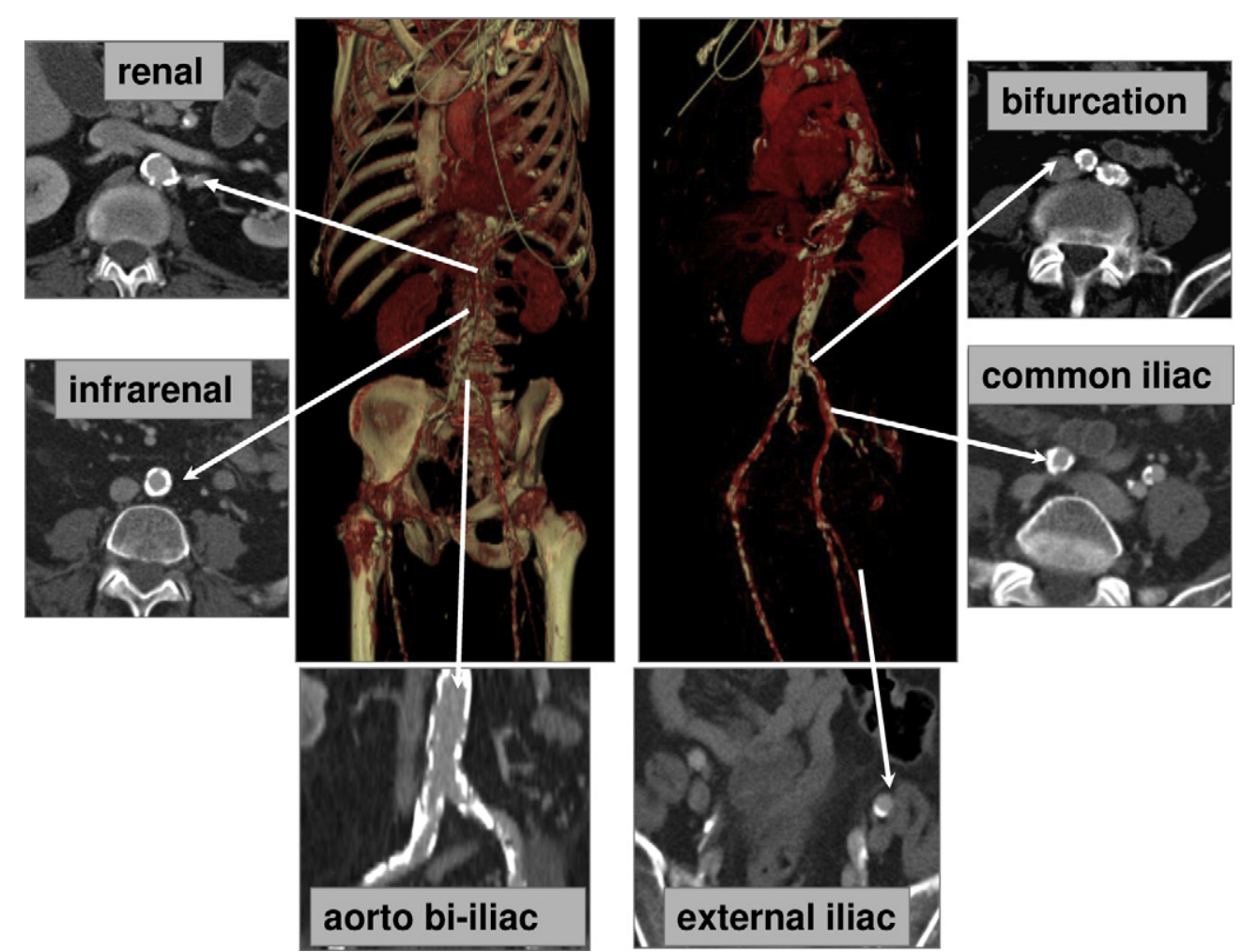

FIGURE 5. Reconstructed multidetector computed tomographic images of the abdominal aorta and its pelvic branches demonstrating tortuosity and extensive calcific atherosclerosis. The extent and degree of peripheral arterial disease is essential in determining the feasibility and safety of transfemoral approaches. In some patients with extensive disease, alternative approaches such as direct aortic, subclavian, or transapical procedures should be considered.

valve (even avoiding the calcium blooming commonly seen on CT) and aortic root can provide a detailed assessment of LV function, aortic annulus, valve, root, and coronary ostia, similar to that obtained on MDCT. ${ }^{188}$ In addition, freebreathing noncontrast navigator-gated 3D whole-heart acquisition can also be obtained to mimic the volumetric acquisition of a CT image. ${ }^{171}$ It also enables assessment of the aortic root in addition to assessing the LVOT-aortic root angulation and predicting imaging planes. The use of $3 \mathrm{D}$ gadolinium-enhanced magnetic resonance imaging can provide precise luminal dimensions of the thoracoabdominal aorta and its iliopelvic branches. ${ }^{189}$ In cases with renal insufficiency, a navigator-gated, free-breathing, 3D noncontrast steady-state free precession sequence can be used to assess luminal dimensions. However, CMR is not optimal for assessment of aortic wall changes, especially dense aortic calcifications, because it would lead to signal voids and hence, appear dark. Postprocedural assessment of residual aortic insufficiency by quantitative CMR might have a potential role in TAVR patients. ${ }^{190}$ However, CMR is a time-intensive technique, which could be a limiting factor, particularly in older patients. In patients with tenuous renal function, the benefits of gadolinium administration have to be balanced against the risks of nephrogenic systemic fibrosis. ${ }^{191}$ In addition to the above-mentioned constraints, CMR is not recommended in patients with pacemakers, defibrillators, or intracranial aneurysm clips, although the currently used valves are CMR compatible.

\subsection{Procedural Performance}

\subsubsection{Role of Surgeon and/or Cardiologist}

The central position of the heart team in optimizing TAVR patient evaluation, procedure performance, and outcomes has been emphasized. Candidacy for TAVR should be determined together by both the surgeon and cardiologist, ideally in an established valve or structural heart disease clinic. During procedural performance, both cardiologist and surgeon should be active participants. There are several specific tasks to be considered among others: (1) gaining access to the vascular tree by either various transarterial sites or by the transapical route; (2) crossing the stenotic aortic valve; (3) balloon aortic valvuloplasty; (4) optimal positioning and deployment of the aortic prosthesis; (5) achieving secure vascular closure; (6) assessment and treatment of proceduralrelated complications, which encompasses vascular access, cardiac structure, coronary artery anatomy, and electrophysiology issues; and (7) considerations for access for hemodynamic support and the need for cardiopulmonary bypass need to be determined by the cardiovascular $(\mathrm{CV})$ surgeon and team. Each of these tasks contains within it multiple component parts, eg, the need for rapid ventricular pacing during 
either balloon aortic valvuloplasty or prosthetic deployment, and identifying the optimal fluoroscopic position to be used.

The above-mentioned tasks often require different skill sets. In the future, as training programs evolve with integration of cardiovascular surgery and interventional cardiology, the roles of either or both specialties may change. However, at the present time and for the foreseeable future, both a surgeon and an interventional cardiologist should be integrally involved with each procedure. Prior to the start of each procedure, a specific team leader should be identified, either the surgeon or the interventional cardiologist. That individual should have overall supervision for the specific case to optimize the procedure. The specific person identified will depend on the operator experience as well as the unique characteristics and challenges of each individual case; for example, the cardiovascular surgeon should be the primary team member responsible for the surgical aspects of transapical and transaortic procedures or if a subclavian cutdown is to be required. Interventional cardiologists usually assume the lead operator position in transfemoral procedures, whereas cardiothoracic surgeons usually lead transapical procedures. The specific roles of the other individuals involved should be identified. Some will be shared, eg, deciding what specific angle identifies the optimal fluoroscopic view for visualizing the plane of the aortic valve for deployment. Other roles will involve just 1 individual, eg, taking the team through the pre-and postprocedure checklists and selection of the specific pacing algorithm for deployment. It is important for all members of the team to be present for all stages of the procedure.

The most important considerations are team-based care, identification of a specific team leader, close communication, and preplanning for outlining management of potential complications. Likewise postprocedure care is optimally delivered on a multispecialty team care service similar to a transplant service where all specialties are participatory to achieve optimal patient outcomes.

\subsubsection{Procedural Location}

Procedural location will vary from institution to institution related to several factors including resources currently available in the facility. The specific location has important physical implications, as well as personnel and equipment implications. Optimal equipment requirements include a state-of-the-art, large-field-of-view fluoroscopic imaging system - preferably a fixed overhead or floor-mounted system that has positioning capability rather than a portable $\mathrm{C}$-arm system. This system needs to have the ability to store and review images and accommodate varying patient sizes. A potentially important adjunct for this is the availability of either biplane imaging or imaging programs that can automatically help aid in the selection of orthogonal views for imaging during positioning of the valve. Integration of TEE echocardiographic images, particularly 3D capabilities, is helpful; the availability of CT or CMR is a significant advantage, particularly if image overlay is possible, which will become more widely used in the future. Full catheterization laboratory hemodynamic capability is also required for hybrid rooms. Other resources required include present cardiopulmonary bypass machines, perfusionists, and related ancillary supplies with an inventory of interventional cardiology equipment for balloon aortic valvuloplasty, coronary balloons, stents, and 0.014-inch wires if coronary occlusion occurs as a complication of device deployment. As vascular access is critical, a variety of peripheral arterial balloons and covered stents for treatment of peripheral vascular complications such as iliac rupture, and a variety of vascular closure devices are also important for completion of the procedure. The procedure location should also be fully capable of providing anesthesia services including advanced airway management, general anesthesia, full hemodynamic monitoring, and administration of vasoactive agents into the central circulation.

As can be seen, these requirements mandate specific room sizes and configurations. Such a hybrid room may be situated in a surgical suite or may be in a large modified catheterization laboratory (approximately $\geq 800$ square feet) with appropriate air handling and air exchange modifications. In the future, as procedures for the treatment of a variety of structural heart and endovascular disease procedures increase, it is anticipated that hybrid rooms will become more standard of care for these team-based therapies.

Personnel requirements are also of great importance. Personnel who are trained to deal with complicated hemodynamics, the specific equipment to be used, and complication management are critical. This has significant implications. For example, if the procedures are carried out in a modified cardiac catheterization laboratory staffed by cardiac laboratory personnel, although there would be expert experience with percutaneous procedures and vascular complications, if urgent cardiopulmonary bypass was required, there may be undue treatment delays related to inexperience with that specific procedure. On the other hand, if the procedures are carried out in an operating room with limited catheterization laboratory capabilities and personnel, the ability to promptly address and treat a coronary or a vascular complication requiring immediate attention may be compromised. Team-based training and care that includes complication management remain a cornerstone.

\subsubsection{Anesthetic Considerations for TAVR}

Patients undergoing TAVR are at a high risk for procedural complications, including hemodynamic collapse. Careful planning and intraoperative anesthetic management can mitigate risk. ${ }^{67,192,193}$ During the preoperative evaluation, special attention is paid to factors that may predict higher risk of intraprocedural instability, in particular: depressed $\mathrm{EF}$, elevated pulmonary pressures, significant MR, incomplete 
revascularization, collateral dependent coronary circulation, COPD, HF, and acute/chronic kidney disease. In patients least likely to tolerate rapid ventricular pacing and hypotension, preventive measures may be instituted ${ }^{194}$ and steps taken to allow for rapid institution of cardiopulmonary bypass. Rarely, elective bypass may be utilized. Routine surgical antibiotic prophylaxis administered prior to surgical incision or vascular access is warranted to decrease the risk of wound infection and endocarditis.

TAVR is typically performed under general anesthesia with central monitoring, using a pulmonary artery catheter and transesophageal echocardiography. Single-lung ventilation is not necessary for TA procedures. Although a temporary ventricular pacing wire can be placed through a hemodynamic catheter, more commonly, a temporary transvenous lead is passed through the femoral or subclavian vein or, in the case of transapical procedures, sewn directly on the epicardial surface. After a ventricular wire is passed, thresholds are checked at a pacing of rate 10 to 20 beats $/ \mathrm{min}$ higher than the patients intrinsic rates. For placement of the CoreValve, rapid pacing for device placement is not required. Arterial pressure monitoring may be done via the radial artery, but in the case of ipsilateral axillary bypass, a plan must be made for additional monitoring either from the contralateral radial or femoral artery. At least 1 largevolume line is obtained peripherally or centrally. Immediate access to a defibrillator device is necessary because ventricular fibrillation can occur with manipulation of catheters within the heart or with rapid ventricular pacing. This may be best accomplished with preapplied defibrillator pads connected to the defibrillator before starting the procedure.

Steps are required to prevent significant hypothermia, and these are often similar to those used in off-pump CABG. The room is heated, fluid warmers are used, and some type of underbody heating system (either forced air or fluid) is generally used. This is important because a limiting step in early extubation of these patients is often the time needed to warm them following the procedure.

Communication in this multidisciplinary approach is the key word for intraoperative success. The importance of training a dedicated team cannot be overemphasized. Frequent changes of personnel will dilute the learning curve. Standard doses of anesthetic, sedative, and narcotic analgesic agents may need to be reduced on the basis of the age and frailty of the patient. ${ }^{195}$ Intraoperative challenges may be encountered even before induction. Use of ultrasound for venous access is beneficial to prevent hemorrhage and complications associated with placement of central line.

Unless otherwise indicated, volume status needs to be supplemented as the patients in this age group are usually volume depleted. Generally, 1.0 L to $1.5 \mathrm{~L}$ of fluid are required, but a combination of pulmonary artery pressures, central venous pressure, and echocardiographic evaluation can guide tailored therapy. Severely underfilled ventricles may pose an additional problem to guidewire/applicator device insertion in these hypertrophied ventricles. Patients with severe concentric LV hypertrophy and intravascular volume depletion may exhibit a rapid and sustained deterioration of hemodynamic status in response to ventricular pacing, rapid ventricular pacing, intracardiac guidewire or catheter manipulations, or balloon aortic valvuloplasty. In patients with low cardiac output and those with more than moderate $\mathrm{PH}$, inotropes such as milrinone or dobutamine may be considered prior to the procedure, with the goal of obtaining a cardiac index of at least $1.8 \mathrm{~L} / \mathrm{min} / \mathrm{m}^{2}$. Inhaled nitric oxide or inhaled epoprostenol should be available for the treatment of severe $\mathrm{PH}$ and right ventricular failure.

Avoidance of prolonged hypotension is perhaps the most important step in preventing hemodynamic collapse. The cycle of hypotension, subendocardial ischemia, low output and further hypotension with ultimate ventricular fibrillation is best avoided as treatment is difficult once these events occur. Maintenance of a mean pressure of $>75 \mathrm{~mm} \mathrm{Hg}$ (or systolic of at least $120 \mathrm{~mm} \mathrm{Hg}$ ) is advisable before initiation of rapid ventricular pacing. The frequency and duration of rapid ventricular pacing episodes may need to be limited to allow enough time between episodes to permit recovery of circulatory function in patients with limited hemodynamic reserve. In patients with a slow recovery of spontaneous circulation after ventricular pacing, pre-emptive therapy with vasopressor therapy such as norepinephrine, epinephrine, or phenylephrine as an intravenous infusion or as incremental intravenous boluses may be important to treat hypotension and facilitate recovery after rapid ventricular pacing. Hypertension may be dangerous and increase the risk of bleeding and ventricular rupture, especially during transapical TAVR.

Anticoagulation therapy is usually initiated after insertion of the regular sheaths and prior to placement of the large sheath into the vasculature, and repeated to maintain an activated clotting time (ACT) of $>300$ seconds. Heparin anticoagulation can be reversed by the administration of protamine sulfate on a milligram to milligram neutralization dose, although it may not be necessary to reverse heparin anticoagulation for transfemoral TAVR if there is a minimal risk of surgical bleeding. A transvenous pacing wire should be secured in position in patients with postprocedural interventricular conduction abnormalities, at high risk for heart block, or with heart block until it is determined whether a permanent pacemaker is necessary. ${ }^{196}$

Although general anesthesia is generally used for transapical procedures, some experienced institutions are performing transfemoral implantation with conscious sedation. ${ }^{197}$ With this approach, with conscious sedation, the patient is awake and spontaneously breathing without an artificial airway. Intraoperative TEE for procedural guidance may be difficult or impossible if TAVR is performed under conscious sedation. Adequacy of ventilation and oxygenation should be continuously assessed during conscious sedation 
and qualified personnel and equipment to perform intubation of the trachea, provide airway protection, and administer mechanical ventilatory support should be immediately available to detect and treat acute respiratory failure in patients managed during TAVR with conscious sedation.

\subsubsection{Vascular Access}

Placement of TAVR is accomplished via femoral artery, subclavian artery, or the aorta. The Sapien valve may be deployed by major transvascular access as well as transapically, whereas the CoreValve uses only major transvascular access. ${ }^{110}$ Careful evaluation of the patient's atherosclerotic load and location, arterial size and tortuosity, and presence of mural thrombus are required for the best possible delivery site. There are specific advantages and disadvantages to each vascular access approach. Selection of the optimal route requires consideration of specific patient anatomy and the specific device to be used.

\subsubsection{Cardiopulmonary Bypass Requirement}

Cardiopulmonary bypass is infrequently $(<5 \%)$ required for support during the valve implantation due to cardiac decompensation as a consequence of cardiac tamponade, coronary occlusion, severe acute AR, aortic rupture, or acute aortic dissection. With experience, and excellent hemodynamic and anesthetic management, this requirement is rarely necessary. Accessory cannulation sites in the femoral vessels or with an adjunctive axillary graft and venous cannula should be considered if femoral access sites are not suitable. Risk of vascular compromise, injury, or particulate embolism should be weighed with the risk of cardiac support needs.

\subsubsection{Percutaneous or Cutdown Access}

Both percutaneous and cutdown access approaches are used; there are advantages and disadvantages to each. Complications with access in this high-risk and generally older population are frequent. Decisions about access technique and site depend on the degree and severity of atherosclerosis, vessel size, specific prosthetic device to be used, and the heart team's experience. ${ }^{136-138,198}$ Use of percutaneous approaches preferentially occurs when access sites are relatively large and free of significant atherosclerotic disease. Less favorable vessels may require cutdown, often with placement of axillary, iliac, or aortic insertion grafts or conduits to provide access sites. Percutaneous insertions are occasionally converted to open repair or hybrid repairs, utilizing percutaneous closure devices and surgical techniques as needed. ${ }^{199}$

\subsubsection{Deployment Technique}

The goals of deployment are to avoid hemodynamic compromise while obtaining a stable valve, positioned without coronary obstruction, interference of mitral valve function, conduction system impingement, or overhanging native aortic leaflets, and avoidance of aortic root complications (rupture and dissection). There are several approaches to the aortic valve, which can be broadly categorized as retrograde or antegrade.

Retrograde passage is generally performed via the femoral artery. There are obvious limitations in patients with peripheral arterial disease or small vessels. Additional, reported retrograde options include the axillary approach and direct ascending aortic puncture. A femoral approach is used in the vast majority of retrograde deployments, starting with either a standard percutaneous femoral arterial access or a surgical exposure of the artery. A series of dilators is employed, under fluoroscopic vision, to reach the size of the deployment sheath. The sheath is passed into the body of the thoracoabdominal aorta. Crossing the aortic valve is accomplished using standard interventional techniques, and a stiff wire exchange is performed, with redundancy in the LV cavity to prevent loss of position. Care must be taken to avoid damage to the $\mathrm{LV}$, resulting in perforation.

The transapical approach is the only currently available antegrade approach, and equipment is only available for this approach for the Sapien valve. Access is obtained via a left anterior thoracotomy, which is made after localization of the apex by fluoroscopy or TTE. After entering the pleural space, digital inspection can further localize the position of the apex and a 2-inch to 3-inch segment of rib may be resected to facilitate exposure. To reduce postoperative pain, soft tissue retractors are preferred to heavy metal retraction. The proper site of puncture is on the left ventricular apex, which is more anterior and proximal than the anatomic cardiac apex. TEE is of great value in helping to localize the apex of the LV. Either 2 concentric purse-string sutures or 2 mattress sutures are placed with felt buttress. Puncture is made and a 0.035 -inch guidewire is passed through the native valve. A balloon catheter may help facilitate wire placement by avoiding the mitral subvalvular apparatus. After the guide wire is placed in the ascending aorta, a coronary catheter such as a JR-4 can be used to guide the wire into the descending aorta, and the wire is exchanged for a stiffer wire (super or extra stiff). The deployment sheath is then passed to a depth of 3 $\mathrm{cm}$ to $4 \mathrm{~cm}$, following which, balloon aortic valvuloplasty of the native valve is performed prior to valve implantation.

A more recent approach that has gained interest and acceptance is the direct aortic or transaortic approach. This approach is being employed with both the balloon expandable and self-expanding techniques. The access is through either an upper partial sternotomy or a second or third right intercostal space minithoracotomy. Concentric felt pledgeted reinforced purse-string sutures are placed in the ascending aorta at least $5 \mathrm{~cm}$ above the valve. A guidewire is then placed retrograde across the valve, and balloon aortic valvuloplasty and valve deployment performed similar to the other access techniques. The advantages of this approach include the short distance from the aortic valve, allowing optimal control and enhanced surgeon comfort level with a technique they are 
already using routinely for cardiopulmonary bypass cannulation as compared with the left ventricular apex approach. Another possible advantage is a less painful incision than with a left anterior thoracotomy at a lower interspace. Disadvantages include manipulation of the ascending aorta with possible embolization of atherosclerotic debris when disease is present. Current-generation delivery systems are being modified for this approach. ${ }^{200}$

\subsubsection{Balloon Expandable Versus Self-Expanding Prostheses}

There are significant differences between the balloonexpandable and self-expanding valves. Balloon-expandable valves, such as the Sapien, cannot be collapsed once expanded. Self-expanding, nitinol-based valves such as the CoreValve can be partially deployed and repositioned to some extent. The promise of recapture and repositioning newer nitinol-based valves offer significant potential advantages in reducing complications from malpositioning. Both valves must be "oversized," but the CoreValve in particular seems to work best when the valve is somewhat underexpanded. Risks of overaggressive sizing include leaflet dysfunction and annular or aortic rupture. ${ }^{110}$

\subsubsection{Imaging During TAVR}

The mainstay of intraprocedural imaging is fluoroscopy and angiography for device placement. TEE is an important adjunct to this and is used at the operator's discretion. The role of intraoperative rotational CT scan is currently in evolution. TEE is used for both transfemoral and transapical deployment, but with transfemoral procedures increasingly being performed under local anesthesia combined with conscious sedation, ${ }^{197}$ the role of TEE in this setting may decrease, though imaging around the time of valve deployment in this setting is possible ${ }^{201}$ and transnasal TEE with smaller probes may allow prolonged monitoring without general anesthesia, though clearly with significant compromise in comparison to standard TEE. TTE may also be used for guidance, though image quality is limited. Similarly, intracardiac echocardiography has been reported for TAVR guidance, though imaging capabilities are much less than TEE. ${ }^{202}$ Personnel performing TEE guidance of TAVR, whether cardiologists or anesthesiologists, must be fully trained in the full spectrum of transesophageal and intraoperative echo with special emphasis on the aortic valve and associated structures. ${ }^{203}$ Training guidelines indicate the need for involvement in 300 intraoperative studies, 150 as an operator. ${ }^{203}$ Additional training is necessary to become familiar with the specifics of TAVR. Frequent changes of the personnel performing TEE guidance dilutes the learning curve and is not recommended. Patients undergoing TAVR tend to be elderly, unstable, and have multiple comorbidities, and thus attention must not be diverted from critical anesthesia management.
It is possible for the attending anesthesiologist to provide echocardiographic procedural guidance, but in many situations, such guidance will need to be provided by a dedicated cardiologist or anesthesiologist not distracted by clinical anesthesia needs.

\subsubsection{Recommendations for TEE Guidance for Patients Undergoing TAVR}

The following brief recommendations can be made for TEE guidance of patients undergoing TAVR. More detailed instructions of procedural echocardiography can be found in a recent expert consensus statement from the American Society of Echocardiography and the European Society of Echocardiography. ${ }^{161}$

1. Guidewire placement: After confirming annular size for proper device selection, TEE can help with guidewire placement. This is particularly important in transapical TAVR, where manual dimpling of the apex can be visualized and guidewire passage through the AV can be confirmed, avoiding the submitral apparatus or the hypertrophied septum.

Valve placement: TEE can be very helpful in the correct placement of the valve prosthesis, though fluoroscopy is commonly used for localization. It is critical to understand the landmarks of the valve when mounted on the guiding catheter. For the Sapien valve, roughly half of the device should be above and below the aortic annulus (Figure 6). For the CoreValve, TEE should confirm that the nitinol stent is well within the borders of the calcified native annulus. Visualizing the valve during the time of rapid pacing and balloon inflation (for the Sapien valve) or deployment of the CoreValve provides an immediate verification of correct valve placement. If the valve is placed using fluoroscopic guidance, the TEE probe must

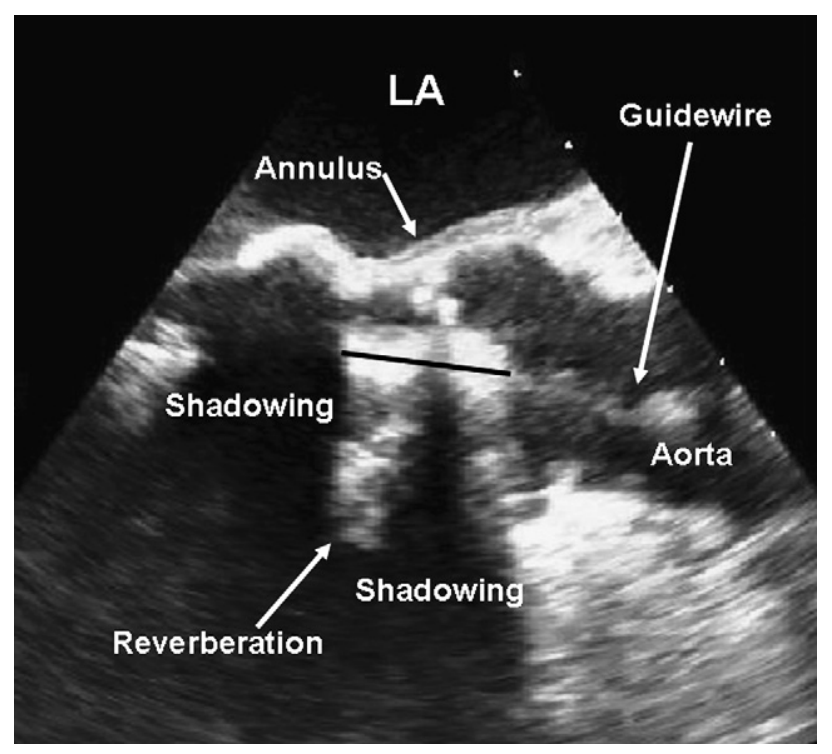

FIGURE 6. Midesophageal long-axis TEE view showing the proper positioning of a sapien valve (black line ${ }^{*}$ ) across the aortic annulus prior to balloon deployment. Note that approximately half the valve is above and below the annulus. Note also the difficulty of imaging due to shadowing from the prosthesis and annular and mitral valve calcification, as well as a prominent reverberation artifact, emphasizing the need for thorough training prior to providing procedural guidance. $L A$, Left atrium. *Visible as black line. 


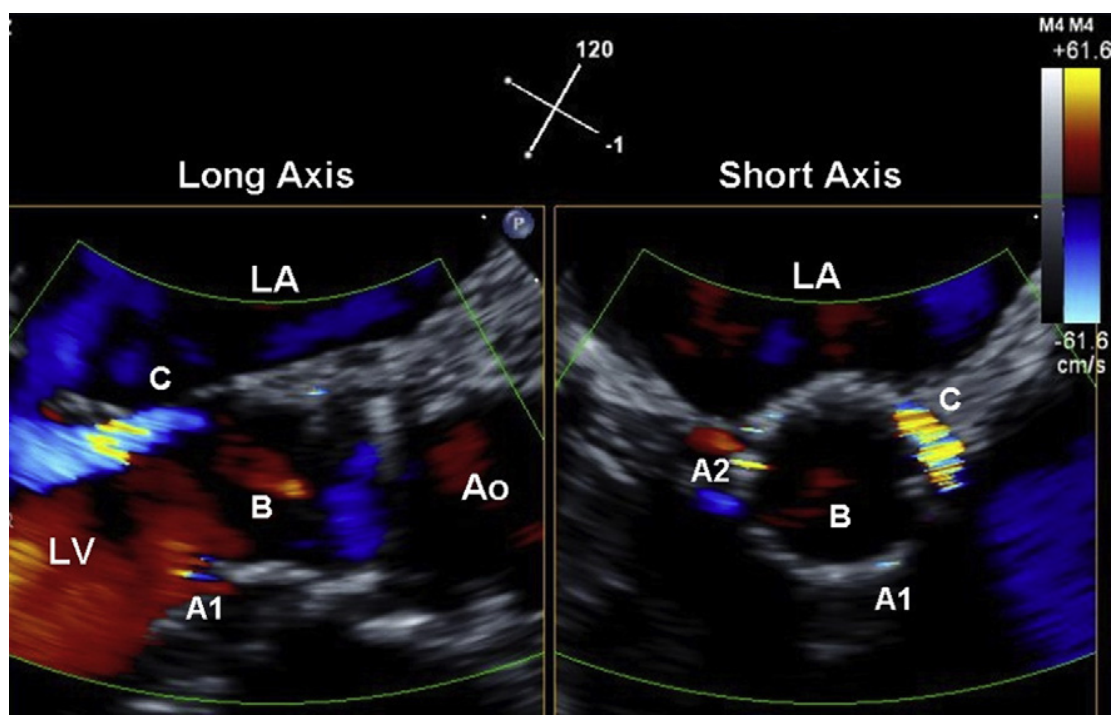

FIGURE 7. Mixed AR. Central and paravalvular AR following TAVR deployment in a biplane long- and short-axis TEE view. $A 1$ and $A 2$ are trivial paravalvular leaks, whereas $B$ is a trivial central regurgitation, all of which are negligible. $C$ is a more severe paravalvular leak, which was ameliorated by a second valve inflation. Ao, Aorta; $A R$, aortic regurgitation; $L A$, left atrium; $L V$, left ventricle.

be partially retracted during that time to facilitate positioning or the fluoroscopic view can be changed.

2. Postdeployment assessment: A particular concern for periprocedural imaging relates to assessment of AR that is complicated by the common frequency of paravalvular leaks and shadowing from the prosthesis (Figures 7 and 8). This assessment must be made very rapidly in the procedure room (to allow possible reballooning or even deployment of a second valve if the AR is severe and cannot be controlled otherwise). It is critical to distinguish between valvular and paravalvular regurgitation and to determine whether it is severe enough to require immediate intervention. Small paravalvular leaks are often visualized due to the widespread irregular calcification in the native valves that leave gaps between the annulus and the prosthesis. If the leaks are punctate in cross section, with jets that do not extend beyond the LVOT and without visible proximal convergence zones above the prosthesis or flow reversal in the aortic arch, then no intervention is needed (Figure 7, jets $\mathrm{A1}$ and $\mathrm{A2}$ ). If not, and velocity aliasing is seen superior to the prosthesis with AR extending beyond the LVOT, then reballooning or a valve-in-valve approach may be appropriate (Figure 7, jet $\mathrm{C}$ ). Mild central valvular regurgitation is commonly seen after valve deployment, which frequently resolves with removal of the guidewire and/or a rise in central aortic pressure (Figure 7, jet B). An inadequately deployed transcatheter valve may have crimped leaflets with more significant valvular $A R$, which dictates reballooning. Rarely, 1 of the leaflets may remain stuck in the open position, resulting in torrential $\mathrm{AR}$ with marked aortic flow reversal and a short pressure half-time (Figure 8). ${ }^{204}$ In such a case, gentle probing with a soft guidewire or catheter may free up the stuck leaflet; if not, immediate placement of a second transcatheter valve should be considered. This will be less of an issue with newer generation valve designs.

Postdeployment echocardiography commonly discloses small areas of paravalvular or central valvular leak. Most commonly, these originate around areas of extreme leaflet calcification, particularly at the commissural areas. If significant, these may be treated with repeat ballooning of the prosthesis to further expand it to close paravalvular leaks or inadequate noncircular deployment. A small additional amount of fluid $(1 \mathrm{~mL})$ may be added to the system prior to reballooning to insure complete inflation. ${ }^{110,205-210}$ For CoreValve, indications are similar-significant paravalvular leak with $A R$ and underexpanded prosthesis (assessed by TEE and/or fluoroscopy). There are a number of other complications that must be recognized immediately after TAVR if poor clinical outcome is to be averted. Persistent hypotension may result from occlusion of a coronary artery by the device or displaced calcium. This can be recognized by characteristic regional hypokinesis, best appreciated from the transgastric view and possibly by evaluating flow in the coronary arteries themselves. Global dysfunction with preserved coronary flow may reflect persistent depression from rapid pacing and balloon inflation, requiring inotropes and possibly intra-aortic counterpulsation or full bypass. Finally, hypotension may result from LVOT obstruction following the abrupt fall in afterload, requiring volume, negative inotropes, and vasopressors. Other etiologies such as severe MR, dislodgement of the AV prosthesis, pericardial tamponade, $\mathrm{RV}$ perforation from the pacemaker lead, air embolism, vascular access bleeding, and aortic dissection must be considered. Although TEE is very helpful for initial device placement and deployment, it is in the setting of hemodynamic instability that TEE is essential to rapidly diagnose these complications.

\subsubsection{Balloon Dilation and Size}

Prior to passage of the valve, predilation of the annulus is performed. Standard techniques of percutaneous balloon aortic valvuloplasty are employed, with rapid pacing during inflation. Radiographic contrast opacification of the root during maximal inflation may provide useful information when the location of the coronary ostia in relation to the annulus and the leaflet calcification of any other aortic root pathology is concerning and requires further delineation. The valvuloplasty balloon size used is generally $20 \mathrm{~mm}$ to 23 $\mathrm{mm}$, depending on the size of the annulus. ${ }^{110,211,212}$

\subsubsection{Rapid Pacing}

Rapid ventricular pacing is generally required for deployments with balloon-expanding devices. The goal is to create 

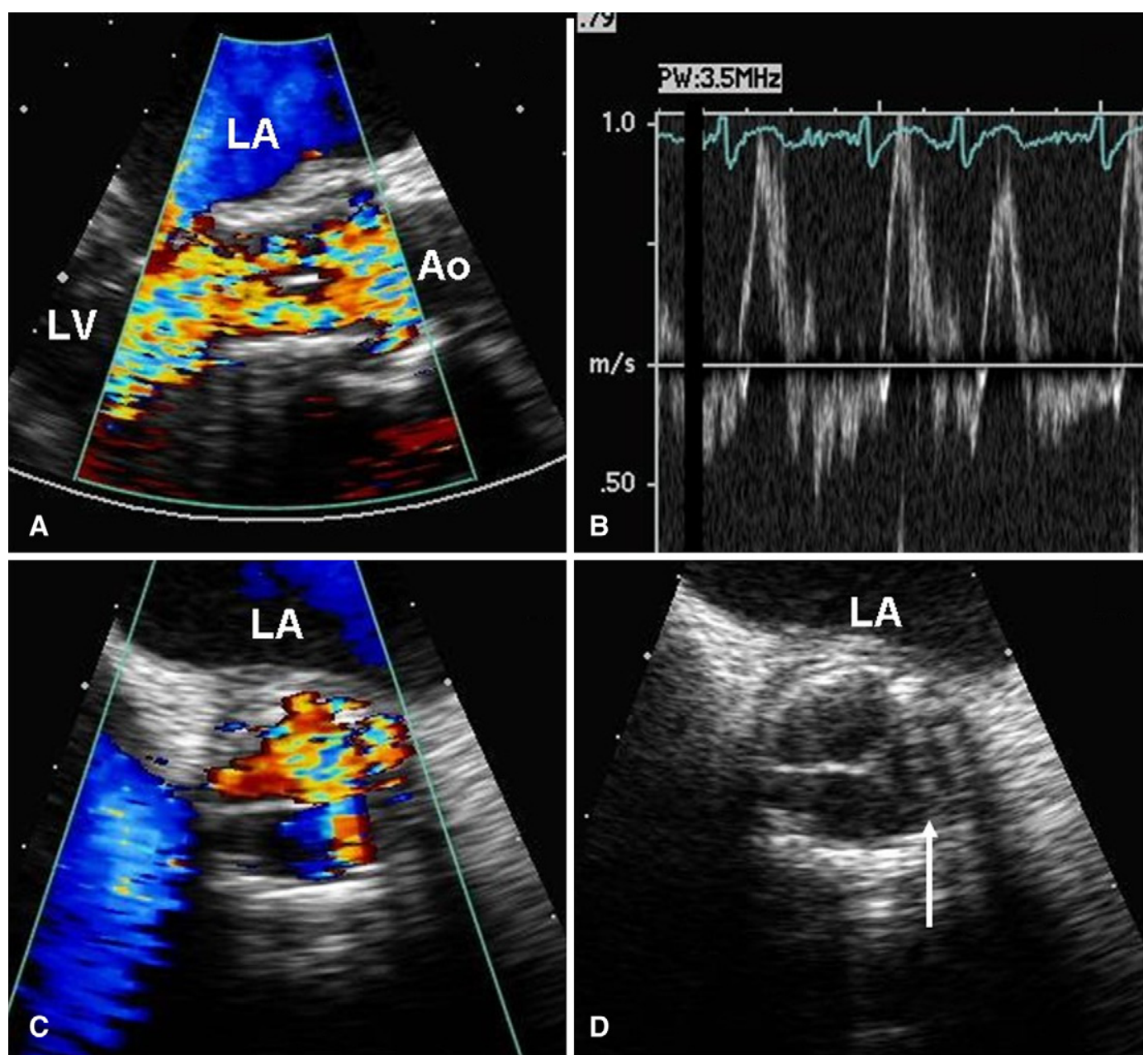

FIGURE 8. Severe AR. Long-axis TEE view showing severe AR following TAVR deployment (A), confirmed by flow reversal in the descending aorta (B). Short axis imaging (C and D) demonstrates that this is due to failure of the leaflet in the left coronary position (arrow) to close in diastole, ultimately treated by deploying a second valve inside this one. Ao, Aorta; $A R$, aortic regurgitation; $L A$, left atrium; $L V$, left ventricle.

a transient reversible decrease in ventricular ejection, thereby reducing forces leading to valve migration during deployment. Typically, this requires pacing at a rate of 160 to 220 beats/min. Ideally, there should be a decrease in systolic pressure to $<70 \mathrm{~mm} \mathrm{Hg}$, with a pulse pressure $<20 \mathrm{~mm} \mathrm{Hg}$. Pacing is accomplished by either transvenous or, less frequently, epicardial electrodes. Reducing the number and duration of pacing runs is important in this tenuous patient population to prevent instability. ${ }^{110,213}$

\subsubsection{Experience With TAVR}

An important issue in evaluating the results of TAVR is standardization of definitions for success, as well as efficacy. The VARC Consortium has proposed the definitions for device success, combined safety endpoint at 30 days, and the combined efficacy endpoint at 1 year or longer (Table 11). ${ }^{96}$ Application of these standard definitions will facilitate comparability and analysis of outcome between different registries and studies. Another important issue on outcomes as well as learning curves relates to the experience of the center, patient selection criteria, and the expert onsite technical support provided by the companies in each case. For example, the UK TAVR experience documented similar results with proctored and nonproctored cases, as well as the first 20 cases versus continued cases in each center. This finding must be considered in light of the fact that the review of potential cases is influenced by the experience that other operators and centers have gained. The presence of the industry representatives in the procedures is also an invaluable asset in recognizing the potential for complications and treatment of same. These 2 background issues enhance the chance of success and may have contributed to the accelerated learning curve. ${ }^{115}$

Compared with patients who underwent TAVR early after its introduction, patients treated more recently have benefited from shared experiences, more careful patient selection, advances in equipment and technique, and expanded operator experience. Prior reports have 
TABLE 11. Composite endpoints

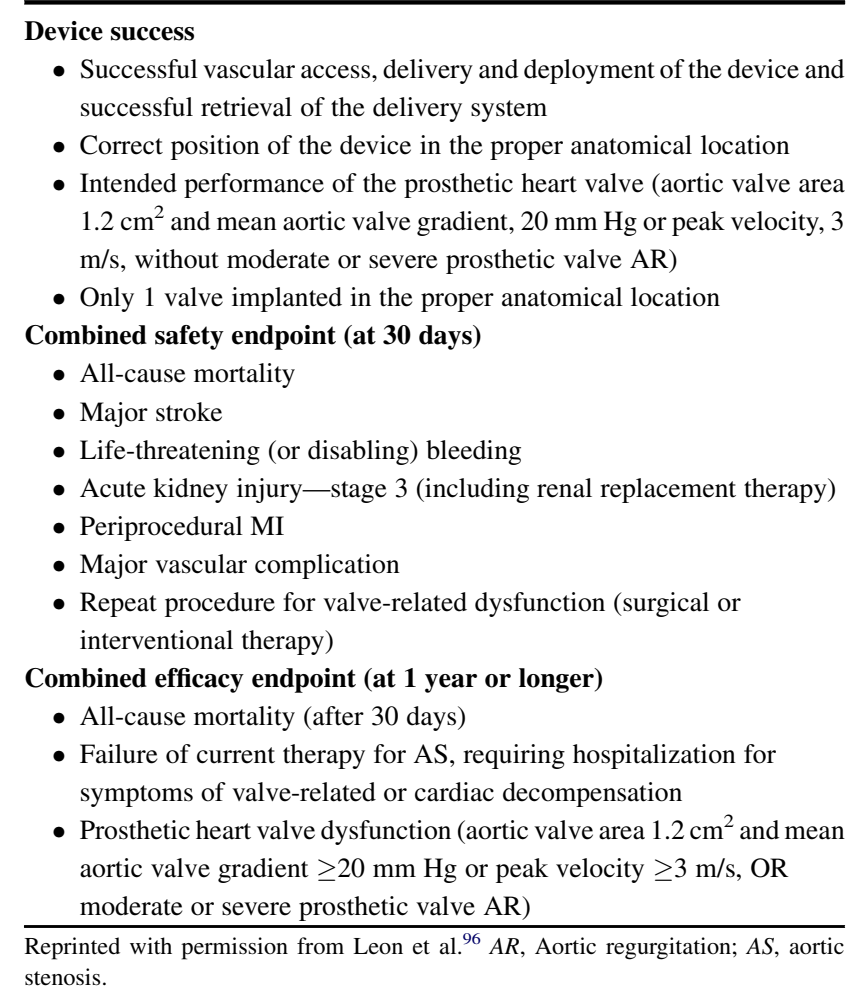

demonstrated a steep learning curve with TAVR using the CoreValve and Sapien valves. ${ }^{214-217}$ Whether improved operator experience alone can improve certain outcomes more than others remains unclear.

Since its introduction, increasing experience with the CoreValve TAVR revealed a trend of improved combined safety endpoint at 30 days from $30 \%$ to $17 \%$, predominately driven by a reduction in life-threatening periprocedural bleeding complications occurring from TAVR. ${ }^{215}$ The most recent UK experience ${ }^{115}$ identified a 30-day mortality of $5.8 \%$. Furthermore, there was a significant reduction in cerebrovascular complications. Despite these trends, all-cause and CV mortality at 1 and 2 years remain high. Experience using the Edwards Sapien valve, on the other hand, revealed that, in 1 analysis, procedural experience was an independent predictor of 30-day mortality. ${ }^{214}$ The overall combined 30-day mortality decreased with increased experience, $10.5 \%$ to $8.5 \%$. Procedural success rates also improved from $92.6 \%$ to $97.8 \%$ with time, predominately in the transfemoral approach, $89.3 \%$ to $98.8 \%$.

Overall, consistent with other percutaneous therapies, the evidence demonstrates that procedural success and mortality rates improve with experience, whereas periprocedural complications are reduced. The introduction of smaller valve platforms introduces some uncertainty in defining whether the improved outcomes are solely related to the operator's experience. Nonetheless operational volumes of given individuals and centers are critical to consider.
Current training protocols attempt to accelerate the learning curve by utilizing experienced proctors and didactic teaching, represented in both the PARTNER and CoreValve trials. If the learning curve can be truncated, procedural outcomes may continue to improve even as more operators enter this clinical arena. Specific criteria regarding operator and center training and experience are the focus of a multisocietal credentialing document. ${ }^{218}$

\subsection{Complication Management}

Complications with TAVR are fairly common due to both the complexity of the procedure, as well as the morbidity of the patients being treated (Table 12). This has led to development of new tools and techniques to manage these complications. $^{219}$

\subsubsection{Shock, Low Cardiac Output Post-TAVR}

The noncompliant hypertrophied ventricles in these patients are very susceptible to myocardial ischemia. The combination of anesthesia, rapid pacing, volume shifts, and brief periods of no cardiac output have made hemodynamic shock and low cardiac output a not infrequent occurrence during and immediately after deployment.

The need for careful management of systemic pressure, inotropic support, and optimal ventilation to avoid and mitigate PH is clear. In patients at extreme risk for hemodynamic instability (ie, those with low EF, collateral dependent coronary circulation, or PH), elective cardiopulmonary bypass $(\mathrm{CPB})$ has been used to facilitate the procedure. Not infrequently, IABP support may be required to bridge these patients to adequate cardiac output. Also rare, but reported, is occlusion of coronary ostia by deployment (approximately $1 \%$ of cases). These may be addressed at times percutaneously, but may require $\mathrm{CPB}$ support for brief periods to allow recovery. Using a combination of these techniques, cardiac failure as a cause of death in TAVR has been rare. ${ }^{109,220-224}$ Rarely, "suicide" LV can occur and must be looked for. This occurs in patients with combined AS and subaortic stenosis or severe LV hypertrophy and cavity obliteration. ${ }^{109,224,225}$ The situation is exacerbated by diuresis and inotropes and is instead managed with volume expansion and beta blockade.

\subsubsection{Annular Rupture}

Annular rupture is a rare but devastating complication of TAVR. Predisposing factors include bulky and dense calcification, small sinotubular junction, smaller annular size, aggressive balloon predilation, and possibly porcelain aorta. Once seen, mortality is high. Management can include decisions for comfort care and sedation, attempts at medical management with pericardial drainage and autotransfusion of smaller leaks, and emergent conversion to open operation, which makes it even more important to define and plan for or against this possibility in patients in a "high-risk" substrate. 226 
TABLE 12. TAVR complications and management

\begin{tabular}{ll}
\hline \multicolumn{1}{c}{ Complication } & Management options depending on the clinical condition, hemodynamic status, and \\
number of options available
\end{tabular}

Each of the management options mentioned should be at least considered in the treatment of these complications. $C A B G$, Coronary artery bypass graft; $C P B$, cardiopulmonary bypass; IABP, intra-aortic balloon pump; $P C I$, percutaneous coronary intervention.

\subsubsection{Post-TAVR Aortic Regurgitation}

Post-TAVR AR must be characterized as to its location, severity, and cause and should integrate both central and paravalvular origins to estimate overall volumetric impact. Central regurgitation is generally a result of improper valve deployment or sizing. Heavy guidewires through the valve can cause a substantial leak by holding a leaflet open, and full evaluation of central leak can only be undertaken once these wires are removed. Overhanging leaflet material can change the diastolic flow pattern and lead to improper leaflet closure. Damage to the leaflets can occur during crimping; significant central AR requires rapid consideration of a valve-in-valve deployment.

Paravalvular leaks of varying degrees are common. These are generally caused by inadequate inflation of the prosthesis or by calcific deposits that prevent the valve unit to properly seat and seal within the annulus. Acute leaks may respond to repeat ballooning the valve to obtain a better seal and more expansion. Predisposing factors include eccentric calcification and heavy irregular calcific deposits within the annular area, and incorrectly sized prostheses. In addition, an increased LVOT angulation in relation to the aorta, and a valve seated less deeply in the annulus predispose to paravalvular leak. Paravalvular regurgitation is quite common immediately post-TAVR, occurring at an incidence of $85 \%$. At 1 year, up to $75 \%$ still have mild or more paravalvular regurgitation, and one third have more than mild regurgitation.

The incidence of residual mild or moderate AR $(+1$ and $+2)$ is significantly higher in TAVR patients compared with surgical procedures, whereas hemodynamically severe postprocedural AR $(+3$ and +4$)$ is rare. Experience has demonstrated that aortic paravalvular regurgitation after self-expanding TAVR devices can be reduced by sufficient balloon aortic valvuloplasty prior to deployment of a percutaneous prosthesis for self-expanding valves. Occasionally in heavily calcified valves, repeat balloon dilation after valve deployment is needed to fully expand the prosthesis. Post-valve dilation can be performed safely with a slightly oversized balloon, without causing significant structural damage to the prosthesis. Appropriate preprocedural planning utilizing both echocardiography and CT for annular analysis is important in order to avoid undersizing of the valve compared with the annulus because this can also cause significant paravalvular regurgitation.

Acute postprocedural regurgitation requires continued surveillance because regurgitation may change in the subsequent days post-TAVR. The self-expanding properties of the stent in the CoreValve prosthesis may reduce the grade of paravalvular AR or recoil from the compressive forces of the heavy calcification on either prosthesis may worsen the degree. $^{227,228}$ Clinical concern about the impact of paravalvular regurgitation after TAVR is prudent, given the findings that after surgical AVR, patients with moderate-severe or severe AR develop chronic volume overload that can lead to left ventricular remodeling/dysfunction and increase the risk for hemolysis. At the present time, there is not sufficient long-term follow-up after TAVR to understand the clinical significance of paravalvular regurgitation and whether the severity of paravalvular 
regurgitation progresses with time. However, it is clear that postprocedural regurgitation is associated with adverse outcomes. ${ }^{109,225}$ There are no reported cases of hemolysis with TAVR despite paravalvular AR.

\subsubsection{Post-TAVR Heart Block}

TAVR is associated with a variable incidence of complete heart block and/or need for pacemaker implantation. ${ }^{132,227-231}$ This is much more common in CoreValve recipients. ${ }^{227-231}$ Preoperative conduction delay is associated with an increased incidence of permanent pacemaker implantation as well. Although heart block occurs usually early after TAVR, it may be delayed up to 30 days. Enhanced surveillance for this complication is important, particularly if the patients are dismissed early following the procedure.

\subsubsection{Post-TAVR Device Migration/Malposition}

Rarely, TAVR devices will become malpositioned or migrate. In the self-expanding models, they can be moved to some degree until fully deployed. The balloon-expandable models do not enjoy this degree of flexibility with positioning. A malpositioned valve may be "unstable" or may embolize. If the valve is unstable, rapid placement of a second overlapping valve may salvage the procedure. Valves embolizing distally may occasionally be extracted in the aorta and a second device implanted. Ventricular embolization requires urgent surgery. ${ }^{185,232,233}$

\subsubsection{Ventricular and Vascular Perforation}

Ventricular perforation is a rare complication of transfemoral TAVR. Its management is similar to that for this complication during percutaneous balloon aortic valvuloplasty, with pericardial drainage and autotransfusion or conversion to open closure. Large-vessel aortoiliac injury similarly is uncommon, but if present, can be managed in most cases by introduction of a covered stent. Some operators place a guidewire down the leg from the contralateral femoral artery for rapid access and control. Readiness for vessel rupture with occlusion balloon cannot be overemphasized. Preparation for this complication is critical with preplanning. It is best managed by avoiding it. Careful maintenance of a good wire position and use of a stiff wire with a soft tip are helpful in prevention of perforation during TAVR. ${ }^{234}$

\subsection{Postprocedural Care}

\subsubsection{Postprocedural Recovery}

Designated units for postprocedural recovery are imperative for optimal care and better outcome of this group of high-risk patients undergoing TAVR. ${ }^{221}$ Although the particulars of postprocedural care will vary from institution to institution and country to country, as well as with the maturity of the TAVR program, the principles of care remain the same that these complex patients should be treated in postprocedural units experienced with both cardiac surgical and interventional cardiology procedures.

\subsubsection{Recommendations for Procedural Care After TAVR}

1. Immediate or early extubation, early mobilization, and meticulous attention to the many potential complications in this elderly, frail group of patients.

2. Post-anesthetic care unit (PACU) or intensive care unit (ICU). There should be a common care pathway with all patients cared for in the same setting so that the care team is conversant with the care pathway. The criteria for weaning in a fast-track concept are as follows:
a. Adequate core temperature of $>36^{\circ} \mathrm{C}$
b. Hemodynamically stable
c. No active bleeding from drainage site

3. The care is somewhat different for transapical and transfemoral patients. Prevention of postoperative hypertension and hypertension upon tracheal extubation is crucial in patients undergoing transapical TAVR to decrease the risk of bleeding or ventricular rupture. Patients undergoing transapical TAVR also require postoperative analgesia for thoracotomy incisional pain and management of thoracostomy tube drainage and subsequent thoracostomy tube removal. Transfemoral TAVR patients require supine positioning until femoral vascular access sheaths are removed and hemostasis has been achieved. Patients undergoing direct aortic and subclavian approaches also need to be carefully monitored for any mediastinal/thoracic bleeding with particular attention paid to avoiding postoperative hypertension.

4. The monitoring includes vital parameters including fluid balance therapy, renal status, and atrioventricular conduction system. Adequate hydration and avoidance of early diuretic administration is important to minimize renal failure. Completion of perioperative surgical antibiotic prophylaxis, resuming preoperative medications such as beta blockers, and initiation of prophylaxis for venous thromboembolism should be addressed within the first 24 hours after operation. A pain management regimen should be initiated immediately if necessary after operation in the postprocedural unit and may consist of intercostal nerve block, infiltration of the wound with local anesthetics, narcotics, or non-narcotic analgesics. Epidural analgesia is not normally required for transapical TAVR because the thoracotomy incision is limited and satisfactory postoperative pain control can be achieved with parenteral or patient-controlled analgesics. Patients undergoing transfemoral TAVR should be evaluated for lower extremity vascular insufficiency, groin hematoma, retroperitoneal bleeding, and femoral artery pseudoaneurysm formation in the instrumented limb.

5. When stable, patients should be transferred to a telemetry unit with hemodynamic and electrocardiographic monitoring capability. The duration of monitoring will depend on the patients response to TAVR and the specific prosthesis used. There are differences in the need for permanent pacemaker implantation between the Edwards Sapien and Medtronic CoreValve device with rates being lower with the former; accordingly, longer electrocardiographic monitoring may be required after implantation of the latter. ${ }^{132} \mathrm{De}-$ pending on institutional protocols, patients should be discharged from the hospital after a final examination with TTE. Antiplatelet therapy with aspirin and clopidogrel is recommended to decrease the risk of thrombotic or thromboembolic complications if there are no contraindications to these medications. ${ }^{235}$

\subsubsection{Hemodynamic Assessment}

Echocardiographic assessment early after TAVR generally reveals a favorable hemodynamic response, with the 
presence of a significantly lower mean transvalvular gradient and a larger effective orifice area (EOA), usually $\leq 10$ $\mathrm{mm} \mathrm{Hg}$ and $>1.5 \mathrm{~cm}^{2}$, respectively. Techniques used for assessment of aortic valve performance vary. The use of measurements of the left of the LVOT diameter and velocity immediately proximal to the stent has been emphasized. ${ }^{236}$ At short- and long-term follow-up, percutaneously implanted valves provide sustained improvements in hemodynamic performance with slightly lower mean transvalvular gradients and larger EOAs than surgically implanted valves, either stented or stentless. ${ }^{237}$

Postprocedure hemodynamic assessment is particularly important to exclude potential prosthesis complications. Transcatheter aortic valves differ, not only in design, but also in implantation technique from surgical AVR. TAVR preserves the native calcified aortic valve compared with surgical AVR, which requires that the native valve be removed prior to prosthesis implantation. The native valve and annular calcification may potentially prevent the adherence of the prosthesis to the aortic wall, whereas low prosthesis deployment into the LV outflow tract may lead to incomplete and/or irregular expansion of the prosthesis, as well as paravalvular prosthetic leak.

An additional concern is severe patient-prosthesis mismatch, which is defined as an effective orifice area index $\leq 0.65 \mathrm{~cm}^{2} / \mathrm{m}^{2} .{ }^{238}$ Patient-prosthesis mismatch in surgical AVR is associated with a reduction in functional status and increased morbidity and mortality at short-term and long-term follow-up. ${ }^{239,240}$ The stent in TAVR prosthesis is thinner than that of the stented valves employed for surgical AVR, minimizing the obstruction to blood flow and reducing the incidence of patient-prosthesis mismatch. Whether reduced rates of patient-prosthesis mismatch will translate to improved functional status and improved survival remains to be defined.

\subsubsection{Atrial Fibrillation}

The incidence of new-onset atrial fibrillation (AF) after successful TAVR ranges from $0.6 \%$ to $8.6 \%{ }^{15,124,132,241}$ However, over $25 \%$ of patients undergoing TAVR have pre-existing AF. Continuous postprocedural electrocardiogram monitoring should be performed for at least 3 days in all patients after transcatheter therapy. Management of atrial fibrillation post-TAVR is based on the ACC/AHA/ ESC guidelines for management of atrial fibrillation. ${ }^{242}$

\subsubsection{Treatment of Stroke}

The treatment of a stroke will depend on the subtype of stroke diagnosed by brain imaging, timing of the event after any procedure, and the severity of the neurological deficit. A large intracerebral hemorrhage could call for reversal of any anticoagulants and correction of any coagulopathy with fresh-frozen plasma or other transfusions. ${ }^{243}$ The diagnosis of an acute ischemic stroke with moderate to severe neurological deficits may require thrombolysis or mechanical clot retrieval depending on the size, location, and whether any major intracerebral artery is occluded. An inhospital stroke within 8 hours of the onset of neurological symptoms usually requires urgent consultation by a stroke neurologist or neurosurgeon. Angiography and stroke intervention may be recommended for large and/or disabling strokes. These treatments are generally available at comprehensive stroke centers. Typical embolic strokes in this setting are the result of calcific emboli. If the stroke is the result of thromboembolism, local rt-PA may be considered although bleeding risk in this elderly population is very high.

Minor strokes, small infarcts on brain imaging, or the absence of any evidence of any major cerebral vessel occlusions can usually be treated with aspirin. ${ }^{244}$ If atrial fibrillation is present, then institution of an oral anticoagulant program is warranted. ${ }^{129}$ If any intracardiac thrombus is detected, then early institution of heparin followed by oral anticoagulants is suggested. ${ }^{63}$ When the patient is stable, other diagnostic evaluations should be done to evaluate for other potential causes of stroke, including vascular imaging to detect any extracranial carotid or vertebral stenosis or intracranial occlusive disease, echocardiography, and Holter monitoring. If no definite source of cardiac embolism is detected, then long-term treatment with antiplatelet options to prevent recurrent stroke may be instituted, which include aspirin, extended-release dipyridamole, and clopidogrel. ${ }^{129}$

\subsection{Long-Term Care}

\subsubsection{Role of Primary Cardiologist}

The long-term care of the patient after TAVR will be mainly guided by the primary referring cardiologist. In contrast to patients undergoing surgical AVR, patients undergoing TAVR tend to be older, with more severe comorbidities and lower functional status, mandating more frequent cardiology follow-up.

The role of the primary cardiologist is to:

1. Prescribe and monitor medical therapy for concurrent cardiovascular disease, including hypertension, atrial fibrillation, heart failure, $\mathrm{CAD}$, and peripheral and cerebrovascular disease, as well as diabetes and dyslipidemia.

2. Prescribe and monitor antithrombotic and/or antiplatelet therapy as recommended for the prosthetic aortic valve and concurrent conditions such as coronary stents or atrial fibrillation.

3. Monitor cardiac and TAVR function with periodic clinical evaluation and echocardiography.

4. Surveillance for and treatment of early and late procedural-related complications

5. Maintain close communication with the implanting physicians if complications occur that may be related to the procedure. 


\subsubsection{Follow-Up Visits}

The patient should be followed closely by the implanting physician team for the first 30 days after TAVR to diagnose and follow any procedural complications. Evaluations should include a post-TAVR baseline echocardiogram and ECG (to document any conduction abnormalities) occurring during that time period. After 30 days, a suggested schedule for follow-up by the primary cardiologist is noted in Table 13.

Follow-up is then continued at the first 6 months, at 1 year, and annually thereafter. The frequency of follow-up evaluations should be increased if there is significant postTAVR paravalvular leak or any change in clinical status or echocardiographic findings. As experience grows with TAVR, the frequency of echocardiography assessment will likely decrease towards that of surgical AVR, deemed appropriate every 3 years. ${ }^{245}$ Additional diagnostic studies may be considered as clinically warranted, including evaluation for coronary disease if symptoms are present.

\subsubsection{Hemodynamic Evaluation}

Hemodynamic evaluation should be performed by echocardiography as indicated in Section 5.5.2 above. Invasive measurement of LV and aortic pressure is not routinely needed unless there are unresolved questions, discrepancies between echocardiographic and physical examination findings, or other unresolved clinical issues.

\subsubsection{Interaction of Co-Treatments}

Given the frequency of coexisting conditions, multiple antiplatelet or anticoagulant strategies may be required. For example, some patients may be receiving antiplatelet therapy for a coronary stent or antithrombotic therapy for atrial fibrillation. This is complicated by the fact that most patients undergoing TAVR are elderly and often have comorbidities that increase bleeding risk. It is prudent to avoid, if possible, the use of multiple anticoagulant therapies. In patients treated with warfarin, a direct thrombin inhibitor, or Factor Xa inhibitor, it is reasonable to continue low-dose aspirin, but other antiplatelet therapy should be avoided, if possible.

\subsubsection{Management of $A R$}

AR after TAVR typically is paravalvular and most often only mild or mild to moderate in severity. At 1-year follow-up, $13 \%$ of patients have no AR, with only trace

TABLE 13. Recommended patient follow-up post-TAVR

\begin{tabular}{lcc}
\hline Timing post-TAVR & Clinical evaluation & Echocardiography \\
\hline 30 days & $\mathrm{X}$ & $\mathrm{X}$ \\
6 months & $\mathrm{X}$ & \\
1 year & $\mathrm{X}$ & $\mathrm{X}$ \\
Annually thereafter & $\mathrm{X}$ & $\mathrm{X}$ \\
\hline
\end{tabular}

$T A V R$, Transcatheter aortic valve replacement. or mild regurgitation in $80 \%$ of patients. ${ }^{15,124}$ These patients do not require any specific therapy for AR other than medical therapy for concurrent hypertension and periodic echocardiography monitoring as described above.

Moderate or severe AR is present after TAVR (even after interventions during the procedure to reduce AR severity) in about $12 \%$ of patients at 30 days and $7 \%$ at 1 -year follow-up. ${ }^{15,124}$

\subsubsection{Recommendations for Managing Severe AR After TAVR}

1 When severe AR is present after TAVR, treatment is similar to native valve AR as detailed in the ACCF/AHA valvular heart disease guidelines. ${ }^{63}$

2 With acute severe AR or chronic severe AR with symptoms of heart failure, surgical AVR may be considered if the patient is a surgical candidate and surgical risk is acceptable. Other options include placement of a second TAVR within the leaking prosthesis ("valve-in-valve").

\section{TECHNOLOGY EVOLUTION}

Next-generation devices promise the potential for improvements, offering expanded clinical utility with advances that include: lower profile delivery catheters, more accurate positioning, reduced paravalvular leak, and ability to either reposition or even retrieve (Table 14). ${ }^{246}$ Many of the new device technologies utilize a selfexpandable, high radial strength repositionable prosthesis consisting of pericardial tissue on a nitinol frame. Two additional valves have recently received approval for commercial sale in Europe. The JenaValve (JenaValve Technology, Munich, Germany) and Acurate Valve (Symetis, Inc., Lausanne, Switzerland) are both delivered currently via a transapical approach.

Other valve designs currently in early clinical studies include Portico Valve (St. Jude Medical, St. Paul, Minn), Direct Flow Medical (Direct Flow Medical, Santa Rosa, Calif), and Sadra Lotus Valve (Sadra Medical, Los Gatos, Calif). Other new designs include flexible sealing membranes aimed at more optimal conformation to the calcified native annulus to reduce paravalvular leaks. New valve designs and materials can also provide the possibility of new prosthesis technology. The Lutter valve was created in an effort to create a more physiological heart valve by utilizing tissue engineering. ${ }^{247}$ The PercValve (Advanced Bioprosthetic Surfaces, San Antonio, Tex) uses nanotechnology in its elastic nitinol frame and leaflets. These leaflets are designed to allow for the growth of endothelial cells, essentially converting it to a tissue valve. Initial animal studies have shown complete endothelialization of the e-nitinol leaflets within 10 days and may eliminate the need for anticoagulation. ${ }^{248} \mathrm{~A}$ final novel approach involves anchoring the prosthesis by using an injectable polymer that cures in position to maintain the implant permanently in place. 
TABLE 14. TAVR valve types and qualities

\begin{tabular}{|c|c|c|c|c|c|c|c|c|c|c|}
\hline Device & Company & $\begin{array}{l}\text { Expansion } \\
\text { mechanism }\end{array}$ & $\begin{array}{c}\text { Valve } \\
\text { material }\end{array}$ & $\begin{array}{c}\text { Stent } \\
\text { material }\end{array}$ & Repositionable & Retrievable & $\begin{array}{c}\text { Clinical } \\
\text { trials }\end{array}$ & FIM & $\begin{array}{c}\text { CE } \\
\text { mark }\end{array}$ & $\begin{array}{l}\text { French } \\
\text { size }\end{array}$ \\
\hline $\begin{array}{l}\text { Colibri Heart } \\
\text { Valve }\end{array}$ & $\begin{array}{l}\text { Endoluminal } \\
\text { Technology } \\
\text { Research }\end{array}$ & $\begin{array}{l}\text { Balloon- and } \\
\text { self-expandable }\end{array}$ & Pericardium & $\begin{array}{l}\text { Stainless } \\
\text { steel/Nitinol }\end{array}$ & No & No & No & 2003 & No & $\begin{array}{l}\text { Balloon: } 16 \\
\text { Self: } 12\end{array}$ \\
\hline Direct Flow & $\begin{array}{l}\text { Direct Flow } \\
\text { Medical }\end{array}$ & Polymer-injected & Pericardium & Polymer & Yes & No & No & 2006 & No & 22 \\
\hline Lotus & Sadra Medical & Self-expandable & Pericardium & Nitinol & Yes & No & No & 2007 & No & 21 \\
\hline JenaValve & $\begin{array}{l}\text { JenaValve } \\
\text { Technology }\end{array}$ & Self-expandable & Pericardium & Nitinol & Yes & No & No & 2007 & Yes & 28 \\
\hline Heart Leaflet & $\begin{array}{l}\text { Heart Leaflet } \\
\text { Technologies }\end{array}$ & Self-expandable & Pericardium & Nitinol & Yes & Yes & No & N/A & No & 16 \\
\hline Lutter & N/A & Self-expandable & $\begin{array}{l}\text { Tissue } \\
\quad \text { engineered }\end{array}$ & Nitinol & No & No & No & N/A & No & N/A \\
\hline PercValve & $\begin{array}{l}\text { Advanced } \\
\quad \text { Bioprosthetic } \\
\text { Surfaces }\end{array}$ & Self-expandable & e-Nitinol & e-Nitinol & No & No & No & N/A & No & 10 \\
\hline Portico & St. Jude & Self-expandable & Pericardium & Nitinol & Yes & Yes & Yes & 2011 & No & 22 \\
\hline Acurate & Symetis & Self-expandable & Pericardium & Nitinol & Yes & Yes & Yes & 2009 & Yes & 28 \\
\hline
\end{tabular}

Modified from Chiam and Ruiz. ${ }^{94} C E$, Conformité Européenne, a mandatory conformity for products placed on the market in the European Economic Area; FIM, first in man; $N /$ $A$, not applicable; TAVR, transcatheter aortic valve replacement.

The outcome with these new technologies will be the focus of multiple registries and then randomized trials.

\section{APPLICATIONS IN NEW PATIENT POPULATIONS AND NEW STUDY DESIGNS}

As experience is gained and technology evolves, the patient population best served with TAVR is likely to change, and 3 areas that merit specific consideration are the "valvein valve" technique for patients with dysfunctional aortic bioprostheses, the use of TAVR in patients with stenotic biscupid aortic valves, and application to lower-risk surgical patients. Other patient populations, such as those with chronic or end-stage renal disease, may also be candidates for this technology and will be the subject of future investigations.

\subsection{Valve-in-Valve}

Patients with dysfunction of a conventional aortic prosthesis present therapeutic challenges. Although repeat operation can be considered, an attractive option is to use a TAVR procedure in which the device is deployed within the previously placed bioprosthesis. ${ }^{209,249-254}$ In multiple small series, transcatheter aortic valve-in-valve implantation appears to be a safe option for the management of bioprosthetic valve failure in patients at high risk for reoperative conventional AVR. In this setting, coronary anatomy should be carefully defined in order to minimize the possibility of coronary obstruction by the transcatheter prosthesis. ${ }^{209,220,250,255}$ In addition, valve-in-valve procedures require a large enough bioprosthetic valve inserted at the index operation to prevent patient prosthetic mismatch with the TAVR valve.

\subsection{Bicuspid Aortic Valve}

The asymmetric valvular anatomy often seen with a bicuspid aortic valve theoretically predisposes to a noncircular expansion of the TAVR device, thereby creating an increased risk of paravalvular regurgitation. ${ }^{256}$

Because of this concern, the presence of a bicuspid aortic valve is considered a relative contraindication to TAVR. Since bicuspid aortic valve patients have generally been excluded from major TAVR trials, there is little clinical experience in this area. Several centers, however, have achieved reasonable success in selected bicuspid aortic valve patients with AS. ${ }^{163,256,257}$ It should be noted that in the Canadian bicuspid aortic valve experience, moderate paravalvular leaks occurred in 2 of 11 patients, and another patient experienced late device migration. ${ }^{256}$ Bicuspid aortic valve patients with bulky leaflets, markedly asymmetric valvular anatomy, and significant aortic incompetence appear to have a higher risk of suboptimal device seating. Whether new valve designs, perhaps those with self-sealing membranes, will improve device performance in this group remains to be determined.

\subsection{Lower-Risk Populations}

Data from the STS Registry indicate that approximately $10 \%$ of patients undergoing AVR have an STS score $\geq 8$ and therefore would be potential candidates for TAVR using current selection criteria. There has been interest in expanding the potential group of candidates for TAVR to include patients with an STS score $\geq 4$. This would broaden to $25 \%$ the number of patients who might be treated with TAVR rather than AVR. There has been concern about the potential for "selection creep," with more lower-risk 
patients treated with TAVR. This should be avoided until more evidence-based data become available on the outcome of TAVR versus AVR in these patients. The planned and ongoing trials evaluating both the Sapien and the CoreValve in lower-risk populations will be of central importance in identifying subsequent utilization of this technology in expanded patient groups.

\section{INTRODUCTION OF TAVR INTO PRACTICE: U.S. VERSUS EUROPEAN PERSPECTIVE}

\subsection{U.S. Perspective}

The U.S. perspective reflects the fact that TAVR approval required a randomized controlled trial, the full extent of which has just now been published. ${ }^{124}$ This contrasts with Europe where 5 new valves or iterations of current valves are already in relatively widespread clinical use. Accordingly, from the U.S. perspective, the rollout of this technology is a key issue. This rollout is influenced by the societal beliefs in a free market; convenient and timely access to medical care; patient and physician expectations; as well as return on investment by companies and institutions alike. These latter issues have led to the proliferation of advanced cardiovascular facilities, which could complicate the rollout of new-device strategies such as TAVR. For example, the state of California alone has 125 facilities that perform percutaneous coronary intervention, the county/city of Los Angeles has 33 cardiovascular surgical and primary STelevation myocardial infarction centers. The Dallas-Fort Worth Metropolitan region also has 33 full-service cardiovascular surgical centers. To plan for each of these centers in either Los Angeles or the Dallas-Fort Worth region to offer TAVR would result in the dilution of concentrated experience. Thus, for a complicated procedure such as TAVR, which is applied in some of the highest-risk patients treated for cardiovascular disease, such as those enrolled in the PARTNER and CoreValve trials, setting up specialized centers of excellence should be a top consideration for optimizing patient care and outcomes.

The concept of development of these specialized heart centers is likely to be somewhat controversial, given the expectation in the United States that each hospital with experienced personnel should be able to perform any and all indicated procedures. However, results of TAVR are likely to be optimal when performed by a heart team of experienced surgeons, structural interventional cardiologists, and CV imaging specialists working together in highvolume tertiary care centers with ancillary support services capable of dealing with very complex patients with advanced comorbid conditions. For detailed recommendations, please refer to the Multisocietal Position Statement on Operator and Institutional Requirements for TVRR. ${ }^{218}$ The specific details of the U.S. rollout and reimbursement for this procedure are as yet to be fully determined. The criteria for regulatory approval and reimbursement by appropriate federal agencies should be based upon expertise; high, adjudicated procedure volumes; and documentation of a health care team approach. In addition, mandatory enrollment in structural heart disease registries should be required so that short- and longer-term outcomes can be assessed and updated with new evolving data.

\subsection{European Perspective}

Adoption of TAVR has been rapid, and the changing trends in Europe have escalated; in selected centers in Germany, TAVR accounts for over $30 \%$ of all AVRs. For example, 1 single center has an experience of more than 1300 TAVRs and has trained more than 360 doctors in over 32 centers from more than 30 countries. Germany itself has approximately 87 centers performing TAVR. The miniaturization of the applicator device, the option to implant newer valves from different manufacturers, and the need for high-quality intraoperative imaging are challenges consistent with the high costs involved with these procedures. Reimbursement varies in different countries. In general, at present, the insurance providers in Europe are bearing the high costs involved in these operative procedures which may be questioned in the future, unless outcomes of improved long-term survival are available. The United Kingdom approach to the development of active TAVR centers has recently been described and can serve as a model for other countries. This rollout included 2 specific technologies (ie, Medtronic CoreValve and Edwards Sapien). The development of this program consisted of didactic session, simulator training, observation of cases at experienced centers, and proctoring at new centers. Core essentials of the program included a multidisciplinary team process for patient selection and for procedural performance. All patients undergoing TAVR were entered into a Central Cardiac Audit Database, which included clinical as well as administrative data using standardized data elements and definitions. This approach has the advantage of including all patients with either of the 2 devices, monitoring the potential of changing patient selection criteria, the ability to document learning curve and the opportunity to evaluate the outcome of patients treated with each of the devices. Particularly relevant findings include the observation that: (1) 30-day and mid-term mortality was equivalent in proctored and nonproctored cases; and (2) the fact that outcomes in the first 20 cases were similar to subsequent cases in each of the 25 centers involved.

\section{ROLE OF REGISTRIES}

Post-marketing data collection for medical device evaluation is an essential component in the assessment of device performance and its benefit/risk balance throughout the product life cycle. In addition to confirmation of data 
observed in pre-approval studies, post-marketing studies provide information regarding 'real world' use in patient subsets not fully tested in pre-market clinical trials. Registries offer an important platform for post-marketing device evaluation. For example, the STS National Database and ACC's National Cardiovascular Data Registry (NCDR) that have traditionally focused on national benchmarking, quality improvement, and research, are rapidly emerging to fulfill this important role. The ability to link clinical registry data with administrative data opens an untapped resource for monitoring and predicting both short- and potentially long-term outcomes. In general, the short-term clinical information can be used for risk stratification and identification of important clinical subgroups, whereas the administrative data can be used to track patterns of use and ultimately long-term events.

In the past, device monitoring has typically been carried out through industry-supported trials and post-approval studies. It has been necessary for industry to undertake the very expensive and time-consuming task of developing data registries. In the development of these registries, there has been little coordination with existing societal registries to harmonize definitions or data specifications. An unfortunate byproduct of this approach has led to the wellrecognized inconsistencies seen in cardiovascular data reporting. In addition, there is the unavoidable potential for a conflict of interest that might arise from a manufacturer conducting studies of its own device.

The use of clinical registries can address many of these concerns. Registries such as the STS Database and ACC's NCDR have a high degree of national participation, so the data represents a true national experience. These registries have a well-established protocol for data collection, with trained abstractors and onsite rigorous audits to ensure high data quality and completeness of entry. Database definitions are generally regarded as national standards, and there is a high degree of harmonization between terms common to these registries. These definitions should be based upon consensus documents; for example, VARC definitions of stroke. ${ }^{96}$ Furthermore, each registry can be linked to administrative databases in order to obtain long-term outcome information. These facts demonstrate the ability of the national cardiovascular registries to serve as the foundation for comprehensive device registries. In the last few years, the FDA has collaborated with various organizations to use registry data to investigate several important devicerelated studies. Although there is no specific legislative mandate to use clinical registries, FDA leadership does actively encourage the use of registries for device surveillance.

Registries offer distinct theoretical advantages in this field, but it should be remembered that practical application presents unchartered waters. Perhaps 1 major challenge lies in the fact that a successful program will require close cooperation between multiple organizations. For example, the STS and ACCF have a long history of cooperation and collaboration on registry-related projects, holding monthly conference calls devoted to registry coordination, thus laying the foundation for collaborative projects such as device surveillance. Similar collaborations will require the need for contractual agreements to address issues such as data ownership, data access, and governance of linked registries. Sophisticated statistical analysis will be a central feature of surveillance projects, so coordination with an analytic center will also be necessary. Certainly, FDA input will be central to any device-related project, and coordination with the Centers for Medicare and Medicaid Services (CMS) may be necessary as well. The device manufacturer will have an essential role, especially in post-approval studies, so coordination with industry will be a key element of any project.

Device surveillance will require fundamental changes in the clinical database operations. Presently, there is no provision for timely data entry, but post-approval studies will require exactly that in order to capture adverse events as they occur. Likewise, post-approval studies require long-term follow-up of information that cannot be obtained from administrative databases. Provision must be made to capture information such as echocardiographic findings and quality-of-life data months to years after device insertion. It should also be noted that the use of CMS MedPAR data may have limited usefulness in this context because of the inability to acquire contemporary data limited to only those patients $>65$ years. (In general, the most current available MedPAR data are 2 years old.) Additional challenges include standardized rigorous methodology adapted for sparse data and standardized reporting formats modeled after the CONSORT (Consolidated Standards of Reporting Trials) guidelines for randomized trials, PRISMA (Preferred Reporting Items for Systematic reviews and Meta-Analyses) guidelines for meta-analyses, or STROBE (STrengthening the Reporting of Observational studies in Epidemiology) guidelines for observational studies. ${ }^{182,258-260}$ It should be emphasized that the registry should capture data on all devices that are placed. Specific post-approval studies may focus on a smaller, more select population, but a comprehensive registry should collect information on all devices for complete analyses independent of industry-sponsored studies. A potential incentive for participation in the device registry might be linking it to CMS coverage criteria or other form of federal legislation.

Funding the device registries requires innovative consideration. Despite the fact that federal agencies encourage the use of clinical registries, funding for such projects is not available for device-related projects. Although industry funding for studies related to their specific product offers the most straightforward approach other 
TABLE 15. Current treatment recommendations for patients with aortic stenosis

\begin{tabular}{|c|c|c|}
\hline Treatment & Indication & Major complications \\
\hline $\begin{array}{l}\text { Surgical aortic valve } \\
\text { replacement }\end{array}$ & $\begin{array}{l}\text { - Symptomatic severe AS (Class I, LOE: B) } \\
\text { - Severe AS undergoing CABG, aortic surgery or other } \\
\text { valve surgery (Class I, LOE: C) } \\
\text { - Symptomatic moderate AS undergoing CABG, aortic } \\
\text { surgery or other valve surgery (Class IIa, LOE: C) } \\
\text { - Asymptomatic severe AS with hypotensive response } \\
\text { to exercise (Class IIb; LOE: C) } \\
\text { - Asymptomatic extremely severe AS (AVA }<0.6 \mathrm{~cm}^{2} \text {, } \\
\text { mean gradient }>50 \mathrm{~mm} \mathrm{Hg} \text {, or jet velocity }>5 \mathrm{~m} / \mathrm{s} \text { ) } \\
\text { (Class IIb, LOE: C) }\end{array}$ & $\begin{array}{l}\text { - } \text { Mortality }(3 \%) \\
\text { - Stroke }(2 \%) \\
\text { - Prolonged ventilation }(11 \%) \\
\text { - Thromboembolism and bleeding } \\
\text { - Prosthetic dysfunction } \\
\text { - Perioperative complications are higher when surgical } \\
\text { AVR is combined with CABG }\end{array}$ \\
\hline $\begin{array}{l}\text { Transcatheter aortic } \\
\text { valve replacement }\end{array}$ & $\begin{array}{l}\text { TAVR is recommended in patients with severe, } \\
\text { symptomatic, calcific stenosis of a trileaflet aortic } \\
\text { valve who have aortic and vascular anatomy suitable } \\
\text { for TAVR and a predicted survival }>12 \text { months, and } \\
\text { who have a prohibitive surgical risk as defined by an } \\
\text { estimated } 50 \% \text { or greater risk of mortality or } \\
\text { irreversible morbidity at } 30 \text { days or other factors such } \\
\text { as frailty, prior radiation therapy, porcelain aorta, and } \\
\text { severe hepatic or pulmonary disease. } \\
\text { - TAVR is a reasonable alternative to surgical AVR in } \\
\text { patients at high surgical risk (PARTNER Trial Criteria: } \\
\text { STS } \geq 8 \% * \text { ) }\end{array}$ & $\begin{array}{l}\text { - Mortality ( } 3 \% \text { to } 5 \%) \\
\text { - Stroke }(6 \% \text { to } 7 \%) \\
\text { - Access complications (17\%) } \\
\text { - Pacemaker insertion } \\
\quad \text { o } 2 \% \text { to } 9 \% \text { (Sapien) } \\
\circ 19 \% \text { to } 43 \% \text { (CoreValve) } \\
\text { - Bleeding } \\
\text { - Prosthetic dysfunction } \\
\text { - Paravalvular AR } \\
\text { - Acute kidney injury } \\
\text { - Other } \\
\text { ○ Coronary occlusion } \\
\text { ○ Valve embolization } \\
\text { ○ Aortic rupture }\end{array}$ \\
\hline Balloon aortic valvuloplasty & $\begin{array}{l}\text { - Reasonable for palliation in adult patients with AS in } \\
\text { whom surgical AVR cannot be performed because of } \\
\text { serious comorbid conditions (Class IIb, LOE: C) } \\
\text { - Bridge to surgical AVR (Class IIb, LOE: C) }\end{array}$ & $\begin{array}{l}\text { - Mortality } \\
\text { - Stroke } \\
\text { - Access complications } \\
\text { - Restenosis }\end{array}$ \\
\hline Medical therapy & $\begin{array}{l}\text { - No specific therapy for asymptomatic AS } \\
\text { - Medical therapy not indicated for symptomatic severe } \\
\text { AS } \\
\text { - Appropriate control of blood pressure and other risk } \\
\text { factors as indicated } \\
\text { - Statins not indicated for preventing progression of AS } \\
\text { - Diuretics, vasodilators and positive inotropes should } \\
\text { be avoided in patients awaiting surgery because of risk } \\
\text { of destabilization }\end{array}$ & - Hemodynamic instability \\
\hline
\end{tabular}

Class I: Conditions for which there is evidence for and/or general agreement that the procedure or treatment is beneficial, useful, and effective; Class II: Conditions for which there is conflicting evidence and/or a divergence of opinion about the usefulness/efficacy of a procedure or treatment; Class IIa: Weight of evidence/opinion is in favor of usefulness/ efficacy; Class IIb: Usefulness/efficacy is less well established by evidence/opinion; LOE B: Data derived from a single randomized trial or nonrandomized studies; LOE C: Only consensus opinion of experts, case studies, or standard-of-care. Source of Class/LOE recommendations: Bonow et al. ${ }^{28} A R$, Aortic regurgitation; $A S$, aortic stenosis; $A V R$, aortic valve replacement; $C A B G$, coronary artery bypass graft; $L O E$, level of evidence; STS, Society of Thoracic Surgeons; TAVR, transcatheter aortic valve replacement. *The original PARTNER protocol specified inclusion criteria as a minimum STS-predicted risk of mortality of $\geq 10$. During the trial enrollment phase, the minimum STS-predicted risk of mortality was changed to $\geq 8$. In both instances, 2 surgeons had to document that the true predicted risk of mortality was $\geq 15$.

models rely upon centers to pay a fee in return for registry participation.

As registries are developed, a "silo" construction must be avoided. It is critically important to keep an eye on the future so that present plans fit seamlessly with the vision for future initiatives. This means that a device-specific registry should be designed to serve as a building block for the next generation of registries. Planning for interoperability, resource sharing, and avoidance of duplication will be necessary to create the system of integrated, coordinated registries that will be the hallmark of registries for the next decade.

\section{SUMMARY AND RECOMMENDATIONS}

There are a number of potential treatment recommendations for patients with AS (Table 15). Consideration of the risk/benefit of each option needs to be carefully evaluated and discussed with the patient and family. The involvement of the heart team in decision making is also essential. 
The approval of TAVR represents a fundamental change in the management of aortic valvular heart disease by offering an alternative to traditional surgical aortic valve replacement in carefully selected patients. The penetration of this technology in the broad group of patients with AS remains to be determined and will depend on the continued evolution of the technology and the results of clinical trials conducted in these patients. At the present time, several observations and recommendations can be made.

1. Complex Technology: Although the technique and equipment continue to evolve, TAVR is a complex procedure with many interlocking steps that require meticulous attention to achieve optimal results and minimize complications.

2. Team-Based Approach: A foundational requirement of TAVR is a team-based approach to patient care. Given the high-risk profile of patients, who often have multiple comorbidities, as well as the technical complexity of the procedure involved, this team-based care will need to include multiple contributors at different stages in the process but will be mainly centered around the primary cardiologist, the cardiovascular surgeon, and the interventional cardiologist. Patients and families must be included in the care team. Other team members will include cardiac anesthesiologists, heart failure specialists, structural heart disease physicians, imaging specialists and the nursing care team, among others.

3. Patient Selection: In adults with severe, symptomatic, calcific stenosis of a trileaflet aortic valve who have aortic and vascular anatomy suitable for TAVR and a predicted survival $>12$ months:

- TAVR is recommended in patients with prohibitive surgical risk.

- TAVR is a reasonable alternative to surgical AVR in patients at high surgical risk.

Definitions of severe AS have varied between registries, trials, and guidelines. In general, all require severely reduced, calcified leaflet motion, and aortic jet velocity $>4.0 \mathrm{~m} / \mathrm{s}$ OR an AVA $<1.0 \mathrm{~cm}^{2}$ OR AV index $<0.6 \mathrm{~cm}^{2} /$ $\mathrm{m}^{2}$ OR a mean gradient $>40 \mathrm{~mm} \mathrm{Hg}$. In the setting of $\mathrm{LV}$ systolic dysfunction, severe AS is present when the leaflets are calcified, with reduced systolic motion, and dobutamine stress echocardiography shows an aortic velocity of $>4.0 \mathrm{~m} /$ s OR mean gradient $>40 \mathrm{~mm} \mathrm{Hg}$ with a valve area $<1.0 \mathrm{~cm}^{2}$ OR AV index $<0.6 \mathrm{~cm}^{2} / \mathrm{m}^{2}$ at any flow rate.

Prohibitive surgical risk is defined as:

- An estimated $50 \%$ or greater risk of mortality or irreversible morbidity at 30 days (as assessed by one cardiologist and 2 cardiothoracic surgeons), or other factors such as frailty, prior radiation therapy, porcelain aorta, and severe hepatic or pulmonary disease.

- Suitable aortic and vascular anatomy is defined as:
- Both aortic annulus size and valve plane to coronary ostium height suitable for placement of an available TAVR.

- Adequate vascular access for passage of the TAVR system (femoral iliac, subclavian, axillary) or suitability for an apical implantation approach.

TAVR is not currently recommended because of limited available information in adults who have:

- An acceptable surgical risk for conventional surgical AVR

- Known bicuspid aortic valve

- Failing bioprosthetic aortic valve

- Severe mitral annular calcification or severe MR

- Moderate AS

- Other (eg, severe AR and subaortic stenosis)

In these groups, additional scientific data will need to be collected to ascertain risk/benefit ratio prior to integration into routine clinical care.

4. TAVR Screening: Screening protocols should be part of every TAVR evaluation. These may vary from institution to institution. Some information may be obtained from referring institutions, whereas some will be obtained within the institution performing the TAVR. In the former case, the information obtained must be of high quality so that optimal recommendations can be formulated. Requisite pieces of information include:

- Data sufficient to calculate STS score

- Measurement of clinical parameters related to the presence of comorbid conditions such as pulmonary function tests in patients with COPD or extent and severity of malignancy if present

- Assessment for the degree of cognitive impairment as appropriate

- Imaging data to confirm

- Presence and severity of aortic stenosis

- Presence and severity of associated CAD

- Left ventricular function

- Presence and severity of associated valvular heart disease lesions

- Presence and extent of cerebral vascular disease

- Preprocedural imaging for planning should be done by the institution performing TAVR

Assessment of annular size for device selection

- Assessment of details of arterial anatomy including the peripheral aortoiliac vessels as well as the aortic arch and ascending aorta which may impact on access selection

5. Site Selection: Centers should have experience with structural heart disease. All members of the heart team should be available onsite. In addition, a structural heart disease center or clinic, a procedural performance area 
(either a hybrid surgical room or a specially modified cardiac catheterization laboratory room), a postprocedure care team, and expert imaging using echocardiography and CT should be available. Setting up specialized centers of excellence with convenient access to patients should be a top priority for responsible dissemination of this technology. For more details, please see facility requirements in the "SCAI/AATS/ACCF/STS Multisociety Expert Consensus Statement: Operator \& Institutional Requirements for Transcatheter Valve Repair and Replacement; Part 1 TAVR.",218

6. Center and Physician Experience: Expertise with surgical AVR is essential-the number of surgical procedures has been recommended to be 50 within the past 12 months. For the interventional cardiologist, experience with balloon aortic valvuloplasty as well as experience in a team-based care approach is recommended. During the rollout of the procedure, experienced proctors will form part of the heart care team. These physicians will be onsite for the first several cases after a site has been initiated. After the performance of these initial procedures, centers will be eligible to qualify for independent TAVR. Physician teams need to be experienced with transapical, transarterial, and alternative arterial approaches for TAVR. For detailed information on requirements for interventional cardiologists and surgeons to perform TAVR, please refer to the "SCAI/ AATS/ACCF/STS Multisociety Expert Consensus Statement: Operator \& Institutional Requirements for Transcatheter Valve Repair and Replacement; Part 1 TAVR." 218

7. Procedural Performance: TAVR should be performed in either specially modified, large cardiac catheterization laboratories or hybrid rooms. Fixed imaging and intraprocedural echocardiography are required as are capabilities for cardiopulmonary bypass for management of procedural complications. In addition to the valve implantation equipment, peripheral and coronary interventions equipment must be available for urgent treatment of complications. The ability to provide general anesthesia should be available.

8. Postprocedural Care: The intensity of postprocedural care depends on the presence of comorbidities, as well as the results of the TAVR itself. Protocols should be defined for routine care, as well as management of specific problems and complications. Ideally, a dedicated recovery area should be established at each site to which all patients should be transferred for optimal postprocedural care. Postdischarge care plans should include consideration of rehabilitation, and home health or other support needed during recovery. In addition, follow-up with the primary care team can ensure successful transitions of care.
9. Registries: Participation is recommended in national TAVR registries that include clinical and administrative claims data that will allow careful evaluation of both short-term and long-term risks and benefits and track changes in patient selection criteria, procedural performance, and device iteration. Preferably, registries should also capture demographics and mortality outcome data of surgically and medically treated patients in order to facilitate comparison of different therapeutic strategies to improve identification and selection of optimal therapeutic options. $^{98-104}$

\section{PRESIDENT AND STAFF}

\section{American College of Cardiology Foundation}

David R. Holmes, Jr, MD, FACC, President

John C. Lewin, MD, Chief Executive Officer

William J. Oetgen, MD, FACC, Senior Vice President, Science and Quality

Charlene L. May, Senior Director, Science and Clinical Policy

Dawn R. Phoubandith, MSW, Director, ACCF Clinical Documents

María Velásquez, Specialist, Clinical Policy and Documents

Erin Barrett, MPS, Senior Specialist, Science and Clinical Policy

\section{References}

1. Holmes DR Jr, Mack MJ. Transcatheter valve therapy a professional society overview from the American College of Cardiology Foundation and the Society of Thoracic Surgeons. J Am Coll Cardiol. 2011;58:445-55.

2. Roberts WC, Ko JM. Frequency by decades of unicuspid, bicuspid, and tricuspid aortic valves in adults having isolated aortic valve replacement for aortic stenosis, with or without associated aortic regurgitation. Circulation. 2005;111:920-5.

3. Selzer A. Changing aspects of the natural history of valvular aortic stenosis. $N$ Engl J Med. 1987;317:91-8.

4. Stephan PJ, Henry AC III, Hebeler RF Jr, et al. Comparison of age, gender, number of aortic valve cusps, concomitant coronary artery bypass grafting, and magnitude of left ventricular-systemic arterial peak systolic gradient in adults having aortic valve replacement for isolated aortic valve stenosis. Am J Cardiol. 1997;79:166-72.

5. Ghaisas NK, Foley JB, O'Briain DS, et al. Adhesion molecules in nonrheumatic aortic valve disease: endothelial expression, serum levels and effects of valve replacement. J Am Coll Cardiol. 2000;36:2257-62.

6. Otto CM, Kuusisto J, Reichenbach DD, et al. Characterization of the early lesion of 'degenerative' valvular aortic stenosis: Histological and immunohistochemical studies. Circulation. 1994;90:844-53.

7. Peterson KL, Tsuji J, Johnson A, et al. Diastolic left ventricular pressurevolume and stress-strain relations in patients with valvular aortic stenosis and left ventricular hypertrophy. Circulation. 1978;58:77-89.

8. Spann JF, Bove AA, Natarajan G, et al. Ventricular performance, pump function and compensatory mechanisms in patients with aortic stenosis. Circulation. 1980;62:576-82.

9. Carabello BA, Paulus WJ. Aortic stenosis. Lancet. 2009;373:956-66.

10. Marcus ML, Doty DB, Hiratzka LF, et al. Decreased coronary reserve: a mechanism for angina pectoris in patients with aortic stenosis and normal coronary arteries. N Engl J Med. 1982;307:1362-6.

11. Koyanagi S, Eastham CL, Harrison DG, et al. Increased size of myocardial infarction in dogs with chronic hypertension and left ventricular hypertrophy. Circ Res. 1982;50:55-62. 
12. Gunther S, Grossman W. Determinants of ventricular function in pressureoverload hypertrophy in man. Circulation. 1979;59:679-88.

13. Krayenbuehl HP, Hess OM, Ritter M, et al. Left ventricular systolic function in aortic stenosis. Eur Heart J. 1988;9(Suppl E):19-23.

14. Carabello BA, Green LH, Grossman W, et al. Hemodynamic determinants of prognosis of aortic valve replacement in critical aortic stenosis and advanced congestive heart failure. Circulation. 1980;62:42-8.

15. Leon MB, Smith CR, Mack M, et al. Transcatheter aortic-valve implantation for aortic stenosis in patients who cannot undergo surgery. $N$ Engl J Med. 2010;363: 1597-607.

16. Gardin JM, Kaplan KJ, Meyers SN, et al. Aortic stenosis: can severity be reliably estimated noninvasively? Chest. 1980;77:130-1.

17. Morrow AG, Roberts WC, Ross J Jr, et al. Obstruction to left ventricular outflow: Current concepts of management and operative treatment. Ann Intern Med. 1968;69:1255-86.

18. Baumgartner H, Hung J, Bermejo J, et al. Echocardiographic assessment of valve stenosis: EAE/ASE recommendations for clinical practice. $J$ Am Soc Echocardiogr. 2009;22:1-23.

19. Popovic AD, Thomas JD, Neskovic AN, et al. Time-related trends in the preoperative evaluation of patients with valvular stenosis. Am J Cardiol. 1997;80: 1464-8.

20. Blais C, Burwash IG, Mundigler G, et al. Projected valve area at normal flow rate improves the assessment of stenosis severity in patients with low-flow, low-gradient aortic stenosis: the multicenter TOPAS (Truly or Pseudo-Severe Aortic Stenosis) study. Circulation. 2006;113:711-21.

21. Grayburn PA. Assessment of low-gradient aortic stenosis with dobutamine. Circulation. 2006;113:604-6.

22. Monin JL, Monchi M, Gest V, et al. Aortic stenosis with severe left ventricular dysfunction and low transvalvular pressure gradients: risk stratification by lowdose dobutamine echocardiography. J Am Coll Cardiol. 2001;37:2101-7.

23. Nishimura RA, Grantham JA, Connolly HM, et al. Low-output, low-gradient aortic stenosis in patients with depressed left ventricular systolic function: the clinical utility of the dobutamine challenge in the catheterization laboratory. Circulation. 2002;106:809-13.

24. Shanewise JS, Cheung AT, Aronson S, et al. ASE/SCA guidelines for performing a comprehensive intraoperative multiplane transesophageal echocardiography examination: recommendations of the American Society of Echocardiography Council for Intraoperative Echocardiography and the Society of Cardiovascular Anesthesiologists Task Force for Certification in Perioperative Transesophageal Echocardiography. Anesth Analg. 1999;89:870-84.

25. Greenland P, Bonow RO, Brundage BH, et al. ACCF/AHA 2007 clinical expert consensus document on coronary artery calcium scoring by computed tomography in global cardiovascular risk assessment and in evaluation of patients with chest pain: a report of the American College of Cardiology Foundation Clinical Expert Consensus Task Force (ACCF/AHA Writing Committee to Update the 2000 Expert Consensus Document on Electron Beam Computed Tomography). J Am Coll Cardiol. 2007;49:378-402.

26. Hundley WG, Bluemke DA, Finn JP, et al. ACCF/ACR/AHA/NASCI/SCMR 2010 expert consensus document on cardiovascular magnetic resonance: a report of the American College of Cardiology Foundation Task Force on Expert Consensus Documents. J Am Coll Cardiol. 2010;55:2614-62.

27. Lancellotti P, Lebois F, Simon M, et al. Prognostic importance of quantitative exercise Doppler echocardiography in asymptomatic valvular aortic stenosis. Circulation. 2005; 112:1377-82.

28. Bonow RO, Carabello BA, Chatterjee K, et al. 2008 focused update incorporated into the ACC/AHA 2006 guidelines for the management of patients with valvular heart disease: a report of the American College of Cardiology/ American Heart Association Task Force on Practice Guidelines (Writing Committee to Revise the 1998 Guidelines for the Management of Patients With Valvular Heart Disease). J Am Coll Cardiol. 2008;52:e1-142.

29. Vahanian A, Baumgartner H, Bax J, et al. Guidelines on the management of valvular heart disease: the Task Force on the Management of Valvular Heart Disease of the European Society of Cardiology. Eur Heart J. 2007;28:230-68.

30. Connolly HM, Oh JK, Orszulak TA, et al. Aortic valve replacement for aortic stenosis with severe left ventricular dysfunction: Prognostic indicators. Circulation. 1997;95:2395-400.

31. Kvidal P, Bergstrom R, Horte LG, et al. Observed and relative survival after aortic valve replacement. J Am Coll Cardiol. 2000;35:747-56

32. Kolh P, Kerzmann A, Honore C, et al. Aortic valve surgery in octogenarians: predictive factors for operative and long-term results. Eur $J$ Cardiothorac Surg. 2007;31:600-6.
33. Litmathe J, Feindt P, Kurt M, et al. Aortic valve replacement in octogenarians: outcome and predictors of complications. Hellenic J Cardiol. 2011;52:211-5.

34. Pereira JJ, Balaban K, Lauer MS, et al. Aortic valve replacement in patients with mild or moderate aortic stenosis and coronary bypass surgery. Am J Med. 2005;118:735-42.

35. Smith WT, Ferguson TB Jr, Ryan T, et al. Should coronary artery bypass graft surgery patients with mild or moderate aortic stenosis undergo concomitant aortic valve replacement?: A decision analysis approach to the surgical dilemma. $J$ Am Coll Cardiol. 2004;44:1241-7.

36. Adams DH, Chikwe J, Filsoufi F, et al. The year in cardiovascular surgery. J Am Coll Cardiol. 2011;57:1425-44.

37. Byrne JG, Leacche M, Unic D, et al. Staged initial percutaneous coronary intervention followed by valve surgery ("hybrid approach") for patients with complex coronary and valve disease. J Am Coll Cardiol. 2005;45:14-8.

38. He GW, Acuff TE, Ryan WH, et al. Aortic valve replacement: determinants of operative mortality. Ann Thorac Surg. 1994;57:1140-6.

39. Pai RG, Varadarajan P, Kapoor N, et al. Aortic valve replacement improves survival in severe aortic stenosis associated with severe pulmonary hypertension. Ann Thorac Surg. 2007;84:80-5.

40. Kapoor N, Varadarajan P, Pai RG. Echocardiographic predictors of pulmonary hypertension in patients with severe aortic stenosis. Eur J Echocardiogr. 2008; 9:31-3.

41. McHenry MM, Rice J, Matlof HJ, et al. Pulmonary hypertension and sudden death in aortic stenosis. Br Heart J. 1979;41:463-7.

42. Rodes-Cabau J, Webb JG, Cheung A, et al. Transcatheter aortic valve implantation for the treatment of severe symptomatic aortic stenosis in patients at very high or prohibitive surgical risk: acute and late outcomes of the multicenter Canadian experience. J Am Coll Cardiol. 2010;55:1080-90.

43. Ben-Dor I, Goldstein SA, Pichard AD, et al. Clinical profile, prognostic implication, and response to treatment of pulmonary hypertension in patients with severe aortic stenosis. Am J Cardiol. 2011;107:1046-51.

44. Cam A, Goel SS, Agarwal S, et al. Prognostic implications of pulmonary hypertension in patients with severe aortic stenosis. J Thorac Cardiovasc Surg. 2011; 142:800-8.

45. Khandhar S, Varadarajan P, Turk R, et al. Survival benefit of aortic valve replacement in patients with severe aortic regurgitation and pulmonary hypertension. Ann Thorac Surg. 2009;88:752-6.

46. Zuern CS, Eick C, Rizas K, et al. Prognostic value of mild-to-moderate pulmonary hypertension in patients with severe aortic valve stenosis undergoing aortic valve replacement. Clin Res Cardiol. 2012;101:81-8.

47. McLaughlin VV, Archer SL, Badesch DB, et al. ACCF/AHA 2009 expert consensus document on pulmonary hypertension: a report of the American College of Cardiology Foundation Task Force on Expert Consensus Documents and the American Heart Association. J Am Coll Cardiol. 2009;53:1573-619.

48. Santini F, Casali G, Franchi G, et al. Hemodynamic effects of inhaled nitric oxide and phosphodiesterase inhibitor (dipyridamole) on secondary pulmonary hypertension following heart valve surgery in adults. Int J Cardiol. 2005;103: 156-63.

49. Fattouch K, Sbraga F, Bianco G, et al. Inhaled prostacyclin, nitric oxide, and nitroprusside in pulmonary hypertension after mitral valve replacement. $J$ Card Surg. 2005;20:171-6.

50. Trachte AL, Lobato EB, Urdaneta F, et al. Oral sildenafil reduces pulmonary hypertension after cardiac surgery. Ann Thorac Surg. 2005;79:194-7.

51. Huber D, Grimm J, Koch R, et al. Determinants of ejection performance in aortic stenosis. Circulation. 1981;64:126-34.

52. Herrmann S, Stork S, Niemann M, et al. Low-gradient aortic valve stenosis myocardial fibrosis and its influence on function and outcome. J Am Coll Cardiol. 2011;58:402-12.

53. Tribouilloy C, Levy F, Rusinaru D, et al. Outcome after aortic valve replacement for low-flow/low-gradient aortic stenosis without contractile reserve on dobutamine stress echocardiography. J Am Coll Cardiol. 2009; 53:1865-73.

54. Quere JP, Monin JL, Levy F, et al. Influence of preoperative left ventricular contractile reserve on postoperative ejection fraction in low-gradient aortic stenosis. Circulation. 2006;113:1738-44.

55. Kolh P, Wijns W, Danchin N, et al. Guidelines on myocardial revascularization. Eur J Cardiothorac Surg. 2010;38. Suppl:S1-52.

56. Toole JM, Stroud MR, Kratz JM, et al. Twenty-five year experience with the St: Jude medical mechanical valve prosthesis. Ann Thorac Surg. 2010;89:1402-9.

57. Braunwald E. Aortic valve replacement: an update at the turn of the millennium. Eur Heart J. 2000;21:1032-3. 
58. Nashef SA, Roques F, Hammill BG, et al. Validation of European System for Cardiac Operative Risk Evaluation (EuroSCORE) in North American cardiac surgery. Eur J Cardiothorac Surg. 2002;22:101-5.

59. Shroyer AL, Coombs LP, Peterson ED, et al. The Society of Thoracic Surgeons: 30-day operative mortality and morbidity risk models. Ann Thorac Surg. 2003; 75:1856-64.

60. Society of Thoracic Surgeons national cardiac surgery database. Accessed January 13, http://www.sts.org/national-database; 2012.

61. O'Brien SM, Shahian DM, Filardo G, et al. The Society of Thoracic Surgeons 2008 cardiac surgery risk models: part 2-isolated valve surgery. Ann Thorac Surg. 2009;88:S23-42.

62. Thourani VH, Ailawadi G, Szeto WY, et al. Outcomes of surgical aortic valve replacement in high-risk patients: a multiinstitutional study. Ann Thorac Surg. 2011;91:49-55.

63. Bonow RO, Carabello BA, Chatterjee K, et al. ACC/AHA 2006 guidelines for the management of patients with valvular heart disease: a report of the American College of Cardiology/American Heart Association Task Force on Practice Guidelines (Writing Committee to Revise the 1998 Guidelines for the Management of Patients With Valvular Heart Disease). J Am Coll Cardiol. 2006;48: e1-148.

64. Melby SJ, Moon MR, Lindman BR, et al. Impact of pulmonary hypertension on outcomes after aortic valve replacement for aortic valve stenosis. J Thorac Cardiovasc Surg. 2011;141:1424-30.

65. Sundermann S, Dademasch A, Praetorius J, et al. Comprehensive assessment of frailty for elderly high-risk patients undergoing cardiac surgery. Eur J Cardiothorac Surg. 2011;39:33-7.

66. Heras M, Chesebro JH, Fuster V, et al. High risk of thromboemboli early after bioprosthetic cardiac valve replacement. J Am Coll Cardiol. 1995;25:1111-9.

67. Brown JM, O'Brien SM, Wu C, et al. Isolated aortic valve replacement in North America comprising 108,687 patients in 10 years: changes in risks, valve types, and outcomes in the Society of Thoracic Surgeons National Database. J Thorac Cardiovasc Surg. 2009;137:82-90.

68. Bloomfield P, Wheatley DJ, Prescott RJ, et al. Twelve-year comparison of a Bjork-Shiley mechanical heart valve with porcine bioprostheses. $N$ Engl J Med. 1991;324:573-9.

69. Goldstein LB, Bushnell CD, Adams RJ, et al. Guidelines for the primary prevention of stroke: a guideline for healthcare professionals from the American Heart Association/American Stroke Association. Stroke. 2011; 42:517-84.

70. Ferrari E, Tozzi P, Hurni M, et al. Primary isolated aortic valve surgery in octogenarians. Eur J Cardiothorac Surg. 2010;38:128-33.

71. Bach DS, Cimino N, Deeb GM. Unoperated patients with severe aortic stenosis. J Am Coll Cardiol. 2007;50:2018-9.

72. Bach DS, Siao D, Girard SE, et al. Evaluation of patients with severe symptomatic aortic stenosis who do not undergo aortic valve replacement: the potential role of subjectively overestimated operative risk. Circ Cardiovasc Qual Outcomes. 2009;2:533-9.

73. Dua A, Dang P, Shaker R, et al. Barriers to surgery in severe aortic stenosis patients with Class I indications for aortic valve replacement. J Heart Valve Dis. 2011;20:396-400.

74. Iung B, Baron G, Butchart EG, et al. A prospective survey of patients with valvular heart disease in Europe: the Euro Heart Survey on Valvular Heart Disease. Eur Heart J. 2003;24:1231-43.

75. Iung B, Cachier A, Baron G, et al. Decision-making in elderly patients with severe aortic stenosis: why are so many denied surgery? Eur Heart J. 2005;26: 2714-20.

76. Varadarajan P, Kapoor N, Bansal RC, et al. Clinical profile and natural history of 453 nonsurgically managed patients with severe aortic stenosis. Ann Thorac Surg. 2006;82:2111-5.

77. Bouma BJ, van Der Meulen JH, van Den Brink RB, et al. Variability in treatment advice for elderly patients with aortic stenosis: a nationwide survey in the Netherlands. Heart. 2001;85:196-201.

78. Varadarajan P, Kapoor N, Bansal RC, et al. Survival in elderly patients with severe aortic stenosis is dramatically improved by aortic valve replacement: results from a cohort of 277 patients aged $>$ or $=80$ years. Eur J Cardiothorac Surg. 2006;30:722-7.

79. Mihaljevic T, Nowicki ER, Rajeswaran J, et al. Survival after valve replacement for aortic stenosis: implications for decision making. J Thorac Cardiovasc Surg. 2008; $135: 1270-8$.

80. Gammie JS, Brown JW, Brown JM, et al. Aortic valve bypass for the high-risk patient with aortic stenosis. Ann Thorac Surg. 2006;81:1605-10.
81. Palta S, Pai AM, Gill KS, et al. New insights into the progression of aortic stenosis: implications for secondary prevention. Circulation. 2000;101: 2497-502.

82. Rajamannan NM, Otto CM. Targeted therapy to prevent progression of calcific aortic stenosis. Circulation. 2004;110:1180-2.

83. Cribier A, Savin T, Saoudi N, et al. Percutaneous transluminal valvuloplasty of acquired aortic stenosis in elderly patients: an alternative to valve replacement? Lancet. 1986;1:63-7.

84. Ben-Dor I, Pichard AD, Satler LF, et al. Complications and outcome of balloon aortic valvuloplasty in high-risk or inoperable patients. J Am Coll Cardiol Intv. 2010;3:1150-6

85. Lieberman EB, Bashore TM, Hermiller JB, et al. Balloon aortic valvuloplasty in adults: failure of procedure to improve long-term survival. J Am Coll Cardiol. 1995;26:1522-8.

86. Otto CM, Mickel MC, Kennedy JW, et al. Three-year outcome after balloon aortic valvuloplasty: Insights into prognosis of valvular aortic stenosis. Circulation. 1994;89:642-50.

87. Percutaneous balloon aortic valvuloplasty: Acute and 30-day follow-up results in 674 patients from the NHLBI Balloon Valvuloplasty Registry. Circulation. 1991;84:2383-97.

88. Ben-Dor I, Maluenda G, Looser PM, et al. Outcomes of concomitant percutaneous coronary intervention and balloon aortic valvuloplasty. Catheter Cardiovasc Interv. 2011. published online before print July 6, 2011, doi:10.1002/ ccd.23193. Accessed January 13, 2012.

89. Hara H, Pedersen WR, Ladich E, et al. Percutaneous balloon aortic valvuloplasty revisited: time for a renaissance? Circulation. 2007;115:e334-8.

90. Ussia GP, Capodanno D, Barbanti M, et al. Balloon aortic valvuloplasty for severe aortic stenosis as a bridge to high-risk transcatheter aortic valve implantation. J Invasive Cardiol. 2010;22:161-6.

91. Chiam PT, Ruiz CE. Percutaneous transcatheter aortic valve implantation: assessing results, judging outcomes, and planning trials: the interventionalist perspective. J Am Coll Cardiol Intv. 2008;1:341-50.

92. Andersen HR, Knudsen LL, Hasenkam JM. Transluminal implantation of artificial heart valves: description of a new expandable aortic valve and initial results with implantation by catheter technique in closed chest pigs. Eur Heart J. 1992;13:704-8

93. Cribier A, Eltchaninoff H, Bash A, et al. Percutaneous transcatheter implantation of an aortic valve prosthesis for calcific aortic stenosis: first human case description. Circulation. 2002;106:3006-8.

94. Chiam PT, Ruiz CE. Percutaneous transcatheter aortic valve implantation: evolution of the technology. Am Heart J. 2009;157:229-42.

95. Dalmau MJ, Gonzalez-Santos JM, Blazquez JA, et al. Hemodynamic performance of the Medtronic Mosaic and Perimount Magna aortic bioprostheses: five-year results of a prospectively randomized study. Eur J Cardiothorac Surg. 2011;39:844-52.

96. Leon MB, Piazza N, Nikolsky E, et al. Standardized endpoint definitions for Transcatheter Aortic Valve Implantation clinical trials: a consensus report from the Valve Academic Research Consortium. J Am Coll Cardiol. 2011;57: 253-69.

97. Avanzas P, Munoz-Garcia AJ, Segura J, et al. Percutaneous implantation of the CoreValve self-expanding aortic valve prosthesis in patients with severe aortic stenosis: early experience in Spain. Rev Esp Cardiol. 2010;63:141-8.

98. Deleted in proof.

99. Deleted in proof.

100. Deleted in proof.

101. Deleted in proof.

102. Deleted in proof

103. Deleted in proof

104. Deleted in proof

105. Buellesfeld L, Gerckens U, Schuler G, et al. 2-year follow-up of patients undergoing transcatheter aortic valve implantation using a self-expanding valve prosthesis. J Am Coll Cardiol. 2011;57:1650-7.

106. Eltchaninoff $\mathrm{H}$, Prat A, Gilard M, et al. Transcatheter aortic valve implantation: early results of the FRANCE (FRench Aortic National CoreValve and Edwards) registry. Eur Heart J. 2011;32:191-7.

107. Godino C, Maisano F, Montorfano M, et al. Outcomes after transcatheter aortic valve implantation with both Edwards-SAPIEN and CoreValve devices in a single center: the Milan experience. J Am Coll Cardiol Intv. 2010;3:1110-21.

108. Moynagh AM, Scott DJ, Baumbach A, et al. CoreValve transcatheter aortic valve implantation via the subclavian artery: comparison with the transfemoral approach. J Am Coll Cardiol. 2011;57:634-5. 
109. Tamburino C, Capodanno D, Ramondo A, et al. Incidence and predictors of early and late mortality after transcatheter aortic valve implantation in 663 patients with severe aortic stenosis. Circulation. 2011;123:299-308.

110. Zahn R, Gerckens U, Grube E, et al. Transcatheter aortic valve implantation: first results from a multi-centre real-world registry. Eur Heart J. 2011;32: 198-204.

111. Thomas M, Schymik G, Walther T, et al. Thirty-day results of the SAPIEN aortic Bioprosthesis European Outcome (SOURCE) Registry: a European registry of transcatheter aortic valve implantation using the Edwards SAPIEN valve. Circulation. 2010;122:62-9.

112. di Marco F, Gerosa G. Percutaneous aortic valve replacement: which patients are suitable for it? A quest for a controlled use. J Thorac Cardiovasc Surg. 2007;133:294-8.

113. ElBardissi AW, Shekar P, Couper GS, et al. Minimally invasive aortic valve replacement in octogenarian, high-risk, transcatheter aortic valve implantation candidates. J Thorac Cardiovasc Surg. 2011;141:328-35.

114. Higgins J, Ye J, Humphries KH, et al. Early clinical outcomes after transapical aortic valve implantation: a propensity-matched comparison with conventional aortic valve replacement. J Thorac Cardiovasc Surg. 2011;142:e47-52.

115. Moat NE, Ludman P, de Belder MA, et al. Long-term outcomes after transcatheter aortic valve implantation in high-risk patients with severe aortic stenosis: the U.K. TAVI (United Kingdom Transcatheter Aortic Valve Implantation) registry. J Am Coll Cardiol. 2011;58:2130-8.

116. Lefevre T, Kappetein AP, Wolner E, et al. One year follow-up of the multicentre European PARTNER transcatheter heart valve study. Eur Heart J. 2011;32:148-57.

117. Krane M, Deutsch MA, Bleiziffer S, et al. Quality of life among patients undergoing transcatheter aortic valve implantation. Am Heart J. 2010;160:451-7.

118. Ussia GP, Mule M, Barbanti M, et al. Quality of life assessment after percutaneous aortic valve implantation. Eur Heart J. 2009;30:1790-6.

119. Bekeredjian R, Krumsdorf U, Chorianopoulos E, et al. Usefulness of percutaneous aortic valve implantation to improve quality of life in patients $>80$ years of age. Am J Cardiol. 2010;106:1777-81.

120. Gotzmann M, Hehen T, Germing A, et al. Short-term effects of transcatheter aortic valve implantation on neurohormonal activation, quality of life and 6minute walk test in severe and symptomatic aortic stenosis. Heart. 2010;96: 1102-6.

121. Wendler O, Walther T, Schroefel H, et al. The SOURCE registry: what is the learning curve in trans-apical aortic valve implantation? Eur J Cardiothorac Surg. 2011;39:853-9.

122. Reynolds MR, Magnuson EA, Lei Y, et al. Health-related quality of life after transcatheter aortic valve replacement in inoperable patients with severe aortic stenosis. Circulation. 2011;124:1964-72.

123. Cohen DJ. Health-related quality of life after transcatheter vs. surgical aortic valve replacement in high-risk patients with severe aortic stenosis. Results from the PARTNER trial (Cohort A). Presented at: TCT 2011; November 10, 2011; San Francisco, CA.

124. Smith CR, Leon MB, Mack MJ, et al. Transcatheter versus surgical aortic-valve replacement in high-risk patients. $N$ Engl J Med. 2011;364:2187-98.

125. Rodes-Cabau J, Dumont E, Boone RH, et al. Cerebral embolism following transcatheter aortic valve implantation: comparison of transfemoral and transapical approaches. J Am Coll Cardiol. 2011;57:18-28.

126. Kahlert P, Knipp SC, Schlamann M, et al. Silent and apparent cerebral ischemia after percutaneous transfemoral aortic valve implantation: a diffusion-weighted magnetic resonance imaging study. Circulation. 2010;121:870-8

127. Arnold M, Schulz-Heise S, Achenbach S, et al. Embolic cerebral insults after transapical aortic valve implantation detected by magnetic resonance imaging. J Am Coll Cardiol Intv. 2010;3:1126-32.

128. Ghanem A, Muller A, Nahle CP, et al. Risk and fate of cerebral embolism after transfemoral aortic valve implantation: a prospective pilot study with diffusionweighted magnetic resonance imaging. J Am Coll Cardiol. 2010;55:1427-32.

129. Furie KL, Kasner SE, Adams RJ, et al. Guidelines for the prevention of stroke in patients with stroke or transient ischemic attack: a guideline for healthcare professionals from the American Heart Association/American Stroke Association. Stroke. 2011;42:227-76.

130. Gutsche JT, Cheung AT, McGarvey ML, et al. Risk factors for perioperative stroke after thoracic endovascular aortic repair. Ann Thorac Surg. 2007;84: 1195-200.

131. Bleiziffer S, Ruge H, Horer J, et al. Predictors for new-onset complete heart block after transcatheter aortic valve implantation. J Am Coll Cardiol Intv. 2010;3:524-30.
132. Piazza N, Onuma Y, Jesserun E, et al. Early and persistent intraventricular conduction abnormalities and requirements for pacemaking after percutaneous replacement of the aortic valve. J Am Coll Cardiol Intv. 2008;1:310-6.

133. Koos R, Mahnken AH, Aktug O, et al. Electrocardiographic and imaging predictors for permanent pacemaker requirement after transcatheter aortic valve implantation. J Heart Valve Dis. 2011;20:83-90.

134. Nuis RJ, Van Mieghem NM, Schultz CJ, et al. Timing and potential mechanisms of new conduction abnormalities during the implantation of the Medtronic CoreValve System in patients with aortic stenosis. Eur Heart J. 2011;32:2067-74.

135. D'Ancona G, Pasic M, Unbehaun A, et al. Permanent pacemaker implantation after transapical transcatheter aortic valve implantation. Interact Cardiovasc Thorac Surg. 2011;13:373-6.

136. Wenaweser P, Pilgrim T, Roth N, et al. Clinical outcome and predictors for adverse events after transcatheter aortic valve implantation with the use of different devices and access routes. Am Heart J. 2011;161:1114-24.

137. Kahlert P, Al-Rashid F, Weber M, et al. Vascular access site complications after percutaneous transfemoral aortic valve implantation. Herz. 2009;34:398-408.

138. Hayashida K, Lefevre T, Chevalier B, et al. Transfemoral aortic valve implantation new criteria to predict vascular complications. J Am Coll Cardiol Intv. 2011;4:851-8.

139. Kodali SK, O'Neill WW, Moses JW, et al. Early and late (one year) outcomes following transcatheter aortic valve implantation in patients with severe aortic stenosis (from the United States REVIVAL trial). Am J Cardiol. 2011;107: 1058-64.

140. Modine T, Obadia JF, Choukroun E, et al. Transcutaneous aortic valve implantation using the axillary/subclavian access: feasibility and early clinical outcomes. J Thorac Cardiovasc Surg. 2011;141:487-91. 491

141. Nuis RJ, Piazza N, Van Mieghem NM, et al. In-hospital complications after transcatheter aortic valve implantation revisited according to the valve academic research consortium definitions. Catheter Cardiovasc Interv. 2011;78: 457-67.

142. Serruys PW, Morice MC, Kappetein AP, et al. Percutaneous coronary intervention versus coronary-artery bypass grafting for severe coronary artery disease. $N$ Engl J Med. 2009;360:961-72.

143. Kempfert J, Van Linden A, Linke A, et al. Transapical aortic valve implantation: therapy of choice for patients with aortic stenosis and porcelain aorta? Ann Thorac Surg. 2010;90:1457-61.

144. Douglas PS, Otto CM, Mickel MC, et al. Gender differences in left ventricle geometry and function in patients undergoing balloon dilatation of the aortic valve for isolated aortic stenosis: NHLBI Balloon Valvuloplasty Registry. Br Heart J. 1995;73:548-54

145. Carroll JD, Carroll EP, Feldman T, et al. Sex-associated differences in left ventricular function in aortic stenosis of the elderly. Circulation. 1992;86: 1099-107

146. Aurigemma GP, Silver KH, McLaughlin M, et al. Impact of chamber geometry and gender on left ventricular systolic function in patients $>60$ years of age with aortic stenosis. Am J Cardiol. 1994;74:794-8.

147. Legget ME, Kuusisto J, Healy NL, et al. Gender differences in left ventricular function at rest and with exercise in asymptomatic aortic stenosis. Am Heart J. 1996;131:94-100.

148. Villari B, Campbell SE, Schneider J, et al. Sex-dependent differences in left ventricular function and structure in chronic pressure overload. Eur Heart J. 1995;16:1410-9.

149. Lunney JR, Lynn J, Foley DJ, et al. Patterns of functional decline at the end of life. JAMA. 2003;289:2387-92.

150. Fried LP, Tangen CM, Walston J, et al, Cardiovascular Health Study Collaborative Research Group. Frailty in older adults: evidence for a phenotype. J Gerontol A Biol Sci Med Sci. 2001;56:M146-56.

151. Himbert D, Descoutures F, Al-Attar N, et al. Results of transfemoral or transapical aortic valve implantation following a uniform assessment in high-risk patients with aortic stenosis. J Am Coll Cardiol. 2009;54:303-11.

152. Afilalo J, Eisenberg MJ, Morin JF, et al. Gait speed as an incremental predictor of mortality and major morbidity in elderly patients undergoing cardiac surgery. J Am Coll Cardiol. 2010;56:1668-76.

153. Consensus statement of the Society of Critical Care Medicine's Ethics Committee regarding futile and other possibly inadvisable treatments. Crit Care Med. 1997;25:887-91.

154. American Thoracic Society. Withholding and withdrawing life-sustaining therapy: This Official Statement of the American Thoracic Society was adopted by the ATS Board of Directors, March 1991. Am Rev Respir Dis. 1991;144: 726-31. 
155. McDermid RC, Bagshaw SM. Prolonging life and delaying death: the role of physicians in the context of limited intensive care resources. Philos Ethics $\mathrm{Hu}$ manit Med. 2009;4:3.

156. Krumholz HM, Merrill AR, Schone EM, et al. Patterns of hospital performance in acute myocardial infarction and heart failure 30-day mortality and readmission. Circ Cardiovasc Qual Outcomes. 2009;2:407-13.

157. Bernheim SM, Grady JN, Lin Z, et al. National patterns of risk-standardized mortality and readmission for acute myocardial infarction and heart failure: update on publicly reported outcomes measures based on the 2010 release. Circ Cardiovasc Qual Outcomes. 2010;3:459-67.

158. Bernheim SM, Spertus JA, Reid KJ, et al. Socioeconomic disparities in outcomes after acute myocardial infarction. Am Heart J. 2007;153:313-9.

159. Rahimi AR, Spertus JA, Reid KJ, et al. Financial barriers to health care and outcomes after acute myocardial infarction. JAMA. 2007;297:1063-72.

160. Smolderen KG, Spertus JA, Reid KJ, et al. The association of cognitive and somatic depressive symptoms with depression recognition and outcomes after myocardial infarction. Circ Cardiovasc Qual Outcomes. 2009;2:328-37.

161. Zamorano JL, Badano LP, Bruce C, et al. EAE/ASE recommendations for the use of echocardiography in new transcatheter interventions for valvular heart disease. J Am Soc Echocardiogr. 2011;24:937-65.

162. Messika-Zeitoun D, Serfaty JM, Brochet E, et al. Multimodal assessment of the aortic annulus diameter: implications for transcatheter aortic valve implantation. J Am Coll Cardiol. 2010;55:186-94.

163. Delgado V, Tops LF, Schuijf JD, et al. Successful deployment of a transcatheter aortic valve in bicuspid aortic stenosis: role of imaging with multislice computed tomography. Circ Cardiovasc Imaging. 2009;2:e12-3.

164. Akhtar M, Tuzcu EM, Kapadia SR, et al. Aortic root morphology in patients undergoing percutaneous aortic valve replacement: evidence of aortic root remodeling. J Thorac Cardiovasc Surg. 2009;137:950-6.

165. Zoghbi WA, Enriquez-Sarano M, Foster E, et al. Recommendations for evaluation of the severity of native valvular regurgitation with two-dimensional and Doppler echocardiography. J Am Soc Echocardiogr. 2003;16:777-802.

166. Ng AC, Delgado V, van der Kley F, et al. Comparison of aortic root dimensions and geometries before and after transcatheter aortic valve implantation by 2 and 3-dimensional transesophageal echocardiography and multislice computed tomography. Circ Cardiovasc Imaging. 2010;3:94-102.

167. Janosi RA, Kahlert P, Plicht B, et al. Measurement of the aortic annulus size by real-time three-dimensional transesophageal echocardiography. Minim Invasive Ther Allied Technol. 2011;20:85-94.

168. Schoenhagen P, Tuzcu EM, Kapadia SR, et al. Three-dimensional imaging of the aortic valve and aortic root with computed tomography: new standards in an era of transcatheter valve repair/implantation. Eur Heart J. 2009;30:2079-86.

169. Al Ali AM, Altwegg L, Horlick EM, et al. Prevention and management of transcatheter balloon-expandable aortic valve malposition. Catheter Cardiovasc Interv. 2008;72:573-8.

170. Schwartz JG, Neubauer AM, Fagan TE, et al. Potential role of threedimensional rotational angiography and $\mathrm{C}$-arm $\mathrm{CT}$ for valvular repair and implantation. Int J Cardiovasc Imaging. 2011;27:1205-22.

171. Koos R, Altiok E, Mahnken AH, et al. Evaluation of aortic root for definition of prosthesis size by magnetic resonance imaging and cardiac computed tomography: implications for transcatheter aortic valve implantation. Int J Cardiol. 2011. published online before print February 9, 2011, doi:10.1016/j.ijcard.2011.01.044. Accessed January 13, 2012.

172. Schoenhagen $P$, Numburi U, Halliburton SS, et al. Three-dimensional imaging in the context of minimally invasive and transcatheter cardiovascular interventions using multi-detector computed tomography: from pre-operative planning to intra-operative guidance. Eur Heart J. 2010;31:2727-40.

173. Chenot F, Montant P, Goffinet C, et al. Evaluation of anatomic valve opening and leaflet morphology in aortic valve bioprosthesis by using multidetector CT: comparison with transthoracic echocardiography. Radiology. 2010;255: 377-85.

174. Earls JP, Berman EL, Urban BA, et al. Prospectively gated transverse coronary $\mathrm{CT}$ angiography versus retrospectively gated helical technique: improved image quality and reduced radiation dose. Radiology. 2008;246:742-53.

175. Feuchtner G, Goetti R, Plass A, et al. Dual-step prospective ECG-triggered 128slice dual-source CT for evaluation of coronary arteries and cardiac function without heart rate control: a technical note. Eur Radiol. 2010;20:2092-9.

176. Bagur R, Webb JG, Nietlispach F, et al. Acute kidney injury following transcatheter aortic valve implantation: predictive factors, prognostic value, and comparison with surgical aortic valve replacement. Eur Heart J. 2010;31: 865-74
177. Joshi SB, Mendoza DD, Steinberg DH, et al. Ultra-low-dose intra-arterial contrast injection for iliofemoral computed tomographic angiography. $J$ Am Coll Cardiol Img. 2009;2:1404-11.

178. Moss RR, Ivens E, Pasupati S, et al. Role of echocardiography in percutaneous aortic valve implantation. J Am Coll Cardiol Img. 2008;1:15-24.

179. Leipsic J, Gurvitch R, Labounty TM, et al. Multidetector computed tomography in transcatheter aortic valve implantation. J Am Coll Cardiol Img. 2011;4: 416-29.

180. O'Brien B, Schoenhagen P, Kapadia SR, et al. Integration of $3 \mathrm{D}$ imaging data in the assessment of aortic stenosis: impact on classification of disease severity. Circ Cardiovasc Imaging. 2011;4:566-73.

181. Pontone G, Andreini D, Bartorelli AL, et al. Feasibility and accuracy of a comprehensive multidetector computed tomography acquisition for patients referred for balloon-expandable transcatheter aortic valve implantation. Am Heart J. 2011;161:1106-13.

182. Schulz KF, Altman DG, Moher D. CONSORT 2010 statement: updated guidelines for reporting parallel group randomized trials. Ann Intern Med. 2010;152: 726-32.

183. Tops LF, Wood DA, Delgado V, et al. Noninvasive evaluation of the aortic roo with multislice computed tomography implications for transcatheter aortic valve replacement. J Am Coll Cardiol Img. 2008;1:321-30.

184. Masson JB, Kovac J, Schuler G, et al. Transcatheter aortic valve implantation: review of the nature, management, and avoidance of procedural complications. J Am Coll Cardiol Intv. 2009;2:811-20.

185. Delgado V, Ng AC, van de Veire NR, et al. Transcatheter aortic valve implantation: role of multi-detector row computed tomography to evaluate prosthesis positioning and deployment in relation to valve function. Eur Heart J. 2010;31: 1114-23.

186. Gurvitch R, Wood DA, Leipsic J, et al. Multislice computed tomography for prediction of optimal angiographic deployment projections during transcatheter aortic valve implantation. J Am Coll Cardiol Intv. 2010;3:1157-65.

187. Kurra V, Kapadia SR, Tuzcu EM, et al. Pre-procedural imaging of aortic roo orientation and dimensions: comparison between $\mathrm{x}$-ray angiographic planar imaging and 3-dimensional multidetector row computed tomography. J Am Coll Cardiol Intv. 2010;3:105-13

188. Jabbour A, Ismail TF, Moat N, et al. Multimodality imaging in transcatheter aortic valve implantation and post-procedural aortic regurgitation: comparison among cardiovascular magnetic resonance, cardiac computed tomography, and echocardiography. J Am Coll Cardiol. 2011;58:2165-73.

189. Yucel EK, Anderson CM, Edelman RR, et al. AHA scientific statement: Magnetic resonance angiography: update on applications for extracranial arteries. Circulation. 1999;100:2284-301

190. Sherif MA, Abdel-Wahab M, Beurich HW, et al. Haemodynamic evaluation of aortic regurgitation after transcatheter aortic valve implantation using cardiovascular magnetic resonance. EuroIntervention. 2011;7:57-63.

191. Juluru K, Vogel-Claussen J, Macura KJ, et al. MR imaging in patients at risk for developing nephrogenic systemic fibrosis: protocols, practices, and imaging techniques to maximize patient safety. Radiographics. 2009;29:9-22.

192. Billings FT, Kodali SK, Shanewise JS. Transcatheter aortic valve implantation anesthetic considerations. Anesth Analg. 2009;108:1453-62.

193. Klein AA, Webb ST, Tsui S, et al. Transcatheter aortic valve insertion: anaesthetic implications of emerging new technology. Br J Anaesth. 2009;103:792-9.

194. Guinot PG, Depoix JP, Etchegoyen L, et al. Anesthesia and perioperative management of patients undergoing transcatheter aortic valve implantation: analysis of 90 consecutive patients with focus on perioperative complications. $J$ Cardiothorac Vasc Anesth. 2010;24:752-61.

195. Burnakis TG, Berman DE. Hostility and hallucinations as a consequence of midazolam administration. DICP. 1989;23:671-2.

196. Latsios G, Gerckens U, Buellesfeld L, et al. "Device landing zone" calcification, assessed by MSCT, as a predictive factor for pacemaker implantation after TAVI. Catheter Cardiovasc Interv. 2010;76:431-9.

197. Fassl J, Seeberger MD, Augoustides JG. Transcatheter aortic valve implantation: is general anesthesia superior to conscious sedation? J Cardiothorac Vasc Anesth. 2011;25:576-7.

198. Kurra V, Schoenhagen P, Roselli EE, et al. Prevalence of significant periphera artery disease in patients evaluated for percutaneous aortic valve insertion: preprocedural assessment with multidetector computed tomography. J Thorac Cardiovasc Surg. 2009; 137:1258-64.

199. Sharp AS, Michev I, Maisano F, et al. A new technique for vascular access management in transcatheter aortic valve implantation. Catheter Cardiovasc Interv. 2010;75:784-93. 
200. Etienne PY, Papadatos S, El Khoury E, et al. Transaortic transcatheter aortic valve implantation with the Edwards SAPIEN valve: feasibility, technical considerations, and clinical advantages. Ann Thorac Surg. 2011;92:746-8.

201. Guarracino F, Cabrini L, Baldassarri R, et al. Non-invasive ventilation-aided transoesophageal echocardiography in high-risk patients: a pilot study. Eur $J$ Echocardiogr. 2010;11:554-6.

202. Bartel T, Bonaros N, Muller L, et al. Intracardiac echocardiography: a new guiding tool for transcatheter aortic valve replacement. J Am Soc Echocardiogr. 2011;24:966-75.

203. Cahalan MK, Stewart W, Pearlman A, et al. American Society of Echocardiography and Society of Cardiovascular Anesthesiologists task force guidelines for training in perioperative echocardiography. J Am Soc Echocardiogr. 2002;15:647-52.

204. Al-Attar N, Himbert D, Vahanian A, et al. Severe intraprosthetic regurgitation by immobile leaflet after trans-catheter aortic valve implantation. Eur J Cardiothorac Surg. 2011;39:591-2.

205. Ussia GP, Sarkar K, Tamburino C. Aortic valve perforation during aortic valvuloplasty: identification and strategies for prevention. Catheter Cardiovasc Interv. 2011;77:876-80.

206. Ussia GP, Barbanti M, Ramondo A, et al. The valve-in-valve technique for treatment of aortic bioprosthesis malposition an analysis of incidence and 1-year clinical outcomes from the Italian CoreValve registry. J Am Coll Cardiol. 2011;57:1062-8.

207. Sherif MA, Abdel-Wahab M, Stocker B, et al. Anatomic and procedural predictors of paravalvular aortic regurgitation after implantation of the Medtronic CoreValve bioprosthesis. J Am Coll Cardiol. 2010;56:1623-9.

208. Latib A, Michev I, Laborde JC, et al. Post-implantation repositioning of the CoreValve percutaneous aortic valve. J Am Coll Cardiol Intv. 2010;3:119-21.

209. Webb JG, Wood DA, Ye J, et al. Transcatheter valve-in-valve implantation for failed bioprosthetic heart valves. Circulation. 2010;121:1848-57.

210. Ruiz CE, Cohen H, Del Valle-Fernandez R, et al. Closure of prosthetic paravalvular leaks: a long way to go. Eur Heart J Suppl. 2010;(12 Suppl E):E52-62.

211. Schultz CJ, Moelker A, Piazza N, et al. Three dimensional evaluation of the aortic annulus using multislice computer tomography: are manufacturer's guidelines for sizing for percutaneous aortic valve replacement helpful? Eur Heart J. 2010;31:849-56.

212. Babaliaros VC, Liff D, Chen EP, et al. Can balloon aortic valvuloplasty help determine appropriate transcatheter aortic valve size? J Am Coll Cardiol Intv 2008;1:580-6.

213. Witzke C, Don CW, Cubeddu RJ, et al. Impact of rapid ventricular pacing during percutaneous balloon aortic valvuloplasty in patients with critical aortic stenosis: should we be using it? Catheter Cardiovasc Interv. 2010;75:444-52.

214. Gurvitch R, Tay EL, Wijesinghe N, et al. Transcatheter aortic valve implantation: lessons from the learning curve of the first 270 high-risk patients. Catheter Cardiovasc Interv. 2011;78:977-84

215. Nuis RJ, Van Mieghem NM, Van der Boon RM, et al. Effect of experience on results of transcatheter aortic valve implantation using a Medtronic CoreValve System. Am J Cardiol. 2011;107:1824-9.

216. Webb JG, Pasupati S, Humphries K, et al. Percutaneous transarterial aortic valve replacement in selected high-risk patients with aortic stenosis. Circulation. 2007; 116:755-63.

217. Webb JG, Altwegg L, Boone RH, et al. Transcatheter aortic valve implantation: impact on clinical and valve-related outcomes. Circulation. 2009;119:3009-16.

218. Tommaso C, Bolman RM, Feldman T, et al. Multisociety expert consensus statement: operator \& institutional requirements for transcatheter valve repair and replacement; part 1 TAVR. J Am Coll Cardiol. 2012 March 1 [E-pub ahead of print]; doi: 10.1016/j.jacc.2012.02.016

219. Himbert D, Roy D, Brecker S, et al. Tools \& techniques: transcatheter aortic valve implantation: transfemoral approach. EuroIntervention. 2011;6:784-5.

220. Gurvitch R, Cheung A, Bedogni F, et al. Coronary obstruction following transcatheter aortic valve-in-valve implantation for failed surgical bioprostheses. Catheter Cardiovasc Interv. 2011;77:439-44.

221. Ussia GP, Scarabelli M, Mule M, et al. Postprocedural management of patients after transcatheter aortic valve implantation procedure with self-expanding bioprosthesis. Catheter Cardiovasc Interv. 2010;76:757-66.

222. Burkhoff D, O'Neill W, Brunckhorst C, et al. Feasibility study of the use of the TandemHeart percutaneous ventricular assist device for treatment of cardiogenic shock. Catheter Cardiovasc Interv. 2006;68:211-7.

223. Maganti MD, Rao V, Borger MA, et al. Predictors of low cardiac output syndrome after isolated aortic valve surgery. Circulation. 2005;112:I448-52.

224. Suh WM, Witzke CF, Palacios IF. Suicide left ventricle following transcatheter aortic valve implantation. Catheter Cardiovasc Interv. 2010;76:616-20.
225. Abdel-Wahab M, Zahn R, Horack M, et al. Aortic regurgitation after transcatheter aortic valve implantation: incidence and early outcome: Results from the German transcatheter aortic valve interventions registry. Heart. 2011;97:899-906.

226. Himbert D, Brochet E, Serfaty JM, et al. Contained aortic root rupture after transcatheter aortic valve implantation. Eur Heart J. 2010;31:2995.

227. Grube E, Laborde JC, Gerckens U, et al. Percutaneous implantation of the CoreValve self-expanding valve prosthesis in high-risk patients with aortic valve disease: the Siegburg first-in-man study. Circulation. 2006;114:1616-24.

228. Grube E, Schuler G, Buellesfeld L, et al. Percutaneous aortic valve replacement for severe aortic stenosis in high-risk patients using the second- and current third-generation self-expanding CoreValve prosthesis: device success and 30day clinical outcome. J Am Coll Cardiol. 2007;50:69-76.

229. Khawaja MZ, Rajani R, Cook A, et al. Permanent pacemaker insertion after CoreValve transcatheter aortic valve implantation: incidence and contributing factors (the UK CoreValve Collaborative). Circulation. 2011;123:951-60.

230. Haworth P, Behan M, Khawaja M, et al. Predictors for permanent pacing after transcatheter aortic valve implantation. Catheter Cardiovasc Interv. 2010;76:751-6.

231. Sinhal A, Altwegg L, Pasupati S, et al. Atrioventricular block after transcatheter balloon expandable aortic valve implantation. J Am Coll Cardiol Intv. 2008;1:305-9.

232. Tuzcu EM. Transcatheter aortic valve replacement malposition and embolization: innovation brings solutions also new challenges. Catheter Cardiovasc Interv. 2008;72:579-80.

233. Tay EL, Gurvitch R, Wijeysinghe N, et al. Outcome of patients after transcatheter aortic valve embolization. J Am Coll Cardiol Intv. 2011;4:228-34.

234. Aminian A, Lalmand J, El Nakadi B. Perforation of the descending thoracic aorta during transcatheter aortic valve implantation (TAVI): an unexpected and dramatic procedural complication. Catheter Cardiovasc Interv. 2011;77: 1076-8.

235. Sun JC, Davidson MJ, Lamy A, et al. Antithrombotic management of patients with prosthetic heart valves: current evidence and future trends. Lancet. 2009; 374:565-76

236. Clavel MA, Rodes-Cabau J, Dumont E, et al. Validation and characterization of transcatheter aortic valve effective orifice area measured by Doppler echocardiography. J Am Coll Cardiol Img. 2011;4:1053-62.

237. Clavel MA, Webb JG, Pibarot P, et al. Comparison of the hemodynamic performance of percutaneous and surgical bioprostheses for the treatment of severe aortic stenosis. J Am Coll Cardiol. 2009;53:1883-91.

238. Pibarot P, Dumesnil JG. Hemodynamic and clinical impact of prosthesis-patient mismatch in the aortic valve position and its prevention. J Am Coll Cardiol. 2000;36:1131-41.

239. Mohty D, Malouf JF, Girard SE, et al. Impact of prosthesis-patient mismatch on long-term survival in patients with small St Jude Medical mechanical prostheses in the aortic position. Circulation. 2006;113:420-6.

240. Blais C, Dumesnil JG, Baillot R, et al. Impact of valve prosthesis-patient mismatch on short-term mortality after aortic valve replacement. Circulation. 2003; 108:983-8

241. Gutierrez M, Rodes-Cabau J, Bagur R, et al. Electrocardiographic changes and clinical outcomes after transapical aortic valve implantation. Am Heart J. 2009; 158:302-8.

242. Fuster V, Ryden LE, Cannom DS, et al. 2011 ACCF/AHA/HRS focused updates incorporated into the ACC/AHA/ESC 2006 guidelines for the management of patients with atrial fibrillation: a report of the American College of Cardiology Foundation/American Heart Association Task Force on Practice Guidelines. $J$ Am Coll Cardiol. 2011;57:e101-98.

243. Morgenstern LB, Hemphill JC III, Anderson C, et al. Guidelines for the management of spontaneous intracerebral hemorrhage: a guideline for healthcare professionals from the American Heart Association/American Stroke Association. Stroke. 2010;41:2108-29.

244. Adams HP Jr, del Zoppo G, Alberts MJ, et al. Guidelines for the early management of adults with ischemic stroke: a guideline from the American Heart Association/American Stroke Association Stroke Council, Clinical Cardiology Council, Cardiovascular Radiology and Intervention Council, and the Atherosclerotic Peripheral Vascular Disease and Quality of Care Outcomes in Research Interdisciplinary Working Groups: the American Academy of Neurology affirms the value of this guideline as an educational tool for neurologists. Stroke. 2007;38:1655-711.

245. Douglas PS, Garcia MJ, Haines DE, et al. ACCF/ASE/AHA/ASNC/HFSA/ HRS/SCAI/SCCM/SCCT/SCMR 2011 appropriate use criteria for echocardiography: A report of the American College of Cardiology Foundation Appropriate Use Criteria Task Force, American Society of Echocardiography, American Heart Association, American Society of Nuclear Cardiology, Heart Failure 
Society of America, Heart Rhythm Society, Society for Cardiovascular Angiography and Interventions, Society of Critical Care Medicine, Society of Cardiovascular Computed Tomography, and Society for Cardiovascular Magnetic Resonance. J Am Coll Cardiol. 2011;57:1126-66.

246. Webb J, Cribier A. Percutaneous transarterial aortic valve implantation: what do we know? Eur Heart J. 2011;32:140-7.

247. Lutter G, Kuklinski D, Berg G, et al. Percutaneous aortic valve replacement: an experimental study: I. Studies on implantation. J Thorac Cardiovasc Surg. 2002; 123:768-76

248. Levi DS, Kusnezov N, Carman GP. Smart materials applications for pediatric cardiovascular devices. Pediatr Res. 2008;63:552-8.

249. Dainese L, Fusari M, Trabattoni P, et al. Redo in aortic homograft replacement: transcatheter aortic valve as a valid alternative to surgical replacement. J Thorac Cardiovasc Surg. 2010;139:1656-7.

250. de Weger A, Ewe SH, Delgado V, et al. First-in-man implantation of a transcatheter aortic valve in a mitral annuloplasty ring: novel treatment modality for failed mitral valve repair. Eur J Cardiothorac Surg. 2011;39:1054-6.

251. Kelpis TG, Mezilis NE, Ninios VN, et al. Minimally invasive transapical aortic valve-in-a-valve implantation for severe aortic regurgitation in a degenerated stentless bioprosthesis. J Thorac Cardiovasc Surg. 2009;138:1018-20.

252. Maroto LC, Rodriguez JE, Cobiella J, et al. Transapical off-pump aortic valvein-a-valve implantation in two elderly patients with a degenerated porcine bioprosthesis. Eur J Cardiothorac Surg. 2010;37:738-40.
253. Rodes-Cabau J, Dumont E, Doyle D, et al. Transcatheter valve-in-valve implantation for the treatment of stentless aortic valve dysfunction. J Thorac Cardiovasc Surg. 2010;140:246-8.

254. Walther T, Kempfert J, Borger MA, et al. Human minimally invasive off-pump valve-in-a-valve implantation. Ann Thorac Surg. 2008;85:1072-3.

255. Mack MJ. Coronary obstruction following transcatheter aortic valve-in-valve implantation for failed surgical bioprostheses. Catheter Cardiovasc Interv. 2011;77:445-6.

256. Wijesinghe N, Ye J, Rodes-Cabau J, et al. Transcatheter aortic valve implantation in patients with bicuspid aortic valve stenosis. J Am Coll Cardiol Intv. 2010; 3:1122-5.

257. Chiam PT, Chao VT, Tan SY, et al. Percutaneous transcatheter heart valve implantation in a bicuspid aortic valve. J Am Coll Cardiol Intv. 2010;3:559-61.

258. Ivers NM, Taljaard M, Dixon S, et al. Impact of CONSORT extension for cluster randomised trials on quality of reporting and study methodology: review of random sample of 300 trials, 2000-8. BMJ. 2011;343:d5886.

259. Liberati A, Altman DG, Tetzlaff J, et al. The PRISMA statement for reporting systematic reviews and meta-analyses of studies that evaluate health care interventions: explanation and elaboration. J Clin Epidemiol. 2009; 62:e1-34.

260. Rios LP, Ye C, Thabane L. Association between framing of the research question using the PICOT format and reporting quality of randomized controlled trials. BMC Med Res Methodol. 2010;10:11.

APPENDIX 1. Author relationships with industry and other entities (relevant)—2012 ACCF/AATS/SCAI/STS expert consensus document on transcatheter aortic valve replacement

\begin{tabular}{|c|c|c|c|c|c|c|c|}
\hline Committee member & Employment & Consultant & $\begin{array}{c}\text { Speaker's } \\
\text { bureau }\end{array}$ & $\begin{array}{c}\text { Ownership/ } \\
\text { partnership/ } \\
\text { principal }\end{array}$ & Personal research & $\begin{array}{c}\text { Institutional, } \\
\text { organizational } \\
\text { or other financial } \\
\text { benefit } \\
\end{array}$ & $\begin{array}{l}\text { Expert } \\
\text { witness }\end{array}$ \\
\hline Arvind Agnihotri* & $\begin{array}{l}\text { Massachusetts General Hospital/ } \\
\text { Harvard Medical School- } \\
\text { Cardiac Surgeon }\end{array}$ & None & None & None & None & $\begin{array}{l}\text { - Edwards } \\
\text { Lifesciences }\end{array}$ & None \\
\hline Karen P. Alexander & $\begin{array}{l}\text { Duke University Medical } \\
\text { Center-Associate Professor } \\
\text { of Medicine/ Cardiology }\end{array}$ & None & None & None & None & None & None \\
\hline Steven R. Bailey* & $\begin{array}{l}\text { University of Texas Medical } \\
\text { Center-Professor of Medicine } \\
\text { and Radiology }\end{array}$ & None & None & None & $\begin{array}{l}\text { - Boston Scientific } \\
\text { (DSMB) } \\
\text { - Palmaz Scientific }\end{array}$ & None & None \\
\hline John H. Calhoon & $\begin{array}{l}\text { University of Texas Health } \\
\text { Sciences Center-Professor } \\
\text { and Chair, Department of CT } \\
\text { Surgery }\end{array}$ & None & None & None & None & None & None \\
\hline Blase A. Carabello* & $\begin{array}{l}\text { Veterans Affairs Medical Center; } \\
\text { Baylor College of Medicine- } \\
\text { Professor of Medicine }\end{array}$ & None & None & None & $\begin{array}{l}\text { - Edwards Lifesciences } \\
(\text { DSMB }) \dagger \\
\text { - Medtronic } \dagger\end{array}$ & None & None \\
\hline Milind Y. Desai & $\begin{array}{l}\text { Cleveland Clinic-Associate } \\
\text { Professor of Medicine, } \\
\text { Department of Cardiovascular } \\
\text { Medicine }\end{array}$ & None & None & None & None & None & None \\
\hline Fred H. Edwards & $\begin{array}{l}\text { University of Florida-Professor } \\
\text { of Surgery; Chief, } \\
\text { Cardiothoracic Surgery }\end{array}$ & None & None & None & None & None & None \\
\hline Gary S. Francis & $\begin{array}{l}\text { University of Minnesota Medical } \\
\text { School—Professor of } \\
\text { Medicine }\end{array}$ & None & None & None & $\begin{array}{l}\text { - Corthera (DSMB) } \\
\text { - Novartis (DSMB) }\end{array}$ & None & None \\
\hline Timothy J. Gardner & $\begin{array}{l}\text { Christiana Care Health } \\
\text { Systems-Medical Director }\end{array}$ & None & None & None & None & None & None \\
\hline David R. Holmes, Jr & $\begin{array}{l}\text { Mayo Clinic-Consultant, } \\
\text { Cardiovascular Diseases }\end{array}$ & None & None & None & None & None & None \\
\hline
\end{tabular}




\begin{tabular}{|c|c|c|c|c|c|c|c|}
\hline Committee member & Employment & Consultant & $\begin{array}{c}\text { Speaker's } \\
\text { bureau }\end{array}$ & $\begin{array}{c}\text { Ownership/ } \\
\text { partnership/ } \\
\text { principal }\end{array}$ & Personal research & $\begin{array}{c}\text { Institutional, } \\
\text { organizational } \\
\text { or other financial } \\
\text { benefit } \\
\end{array}$ & $\begin{array}{l}\text { Expert } \\
\text { witness } \\
\end{array}$ \\
\hline A. Pieter Kappetein* & $\begin{array}{l}\text { Erasmus Medical Center- } \\
\text { Department of Thoracic } \\
\text { Surgery }\end{array}$ & None & None & None & None & $\begin{array}{l}\text { - } \text { Boston } \\
\text { Scientific } \dagger \\
\text { - } \text { Medtronic } \dagger\end{array}$ & None \\
\hline Sanjay Kaul & $\begin{array}{l}\text { Cedars-Sinai Medical Center- } \\
\text { Director, Cardiology } \\
\text { Fellowship Training Program }\end{array}$ & None & None & None & None & None & None \\
\hline Jane A. Linderbaum & $\begin{array}{l}\text { Mayo Clinic; Saint Mary’s } \\
\text { Hospital—Assistant Professor } \\
\text { of Medicine; Director, CV } \\
\text { Inpatient Practice }\end{array}$ & None & None & None & None & None & None \\
\hline Michael J. Mack* & $\begin{array}{l}\text { The Heart Hospital Baylor } \\
\text { Plano-Director }\end{array}$ & None & None & None & $\begin{array}{l}\text { - } \text { Boston Scientific } \dagger \\
\text { - Edwards } \\
\text { Lifesciences } \dagger\end{array}$ & None & None \\
\hline Chirojit Mukherjee & $\begin{array}{l}\text { Heart Center Leipzig University } \\
\text { of Leipzig, Germany-Senior } \\
\text { Consultant \& Director } \\
\text { Fellowship Program } \\
\text { Anesthesia and Intensive } \\
\text { Medicine II }\end{array}$ & None & None & None & None & None & None \\
\hline Debabrata Mukherjee & $\begin{array}{l}\text { Texas Tech University Health } \\
\text { Sciences Center-Chief, } \\
\text { Cardiovascular Medicine }\end{array}$ & None & None & None & None & None & None \\
\hline Catherine M. Otto & $\begin{array}{l}\text { University of Washington, } \\
\text { Division of Cardiology- } \\
\text { Professor of Medicine }\end{array}$ & None & None & None & None & None & None \\
\hline Carlos E. Ruiz & $\begin{array}{l}\text { Lenox Hill Heart and Vascular } \\
\text { Institute of New York- } \\
\text { Professor and Chief, Division } \\
\text { of Pediatric Cardiology }\end{array}$ & None & None & None & None & None & None \\
\hline Ralph L. Sacco & $\begin{array}{l}\text { Miller School of Medicine, } \\
\text { Jackson Memorial Hospital, } \\
\text { University of Miami- } \\
\text { Chairman of Neurology, } \\
\text { Professor of Neurology, Public } \\
\text { Health \& Epidemiology, Human } \\
\text { Genetics, and Neurosurgery; } \\
\text { Executive Director, McKnight } \\
\text { Brain Institute }\end{array}$ & None & None & None & None & None & None \\
\hline Donnette Smith & $\begin{array}{l}\text { Mended Hearts-Consumer } \\
\text { Advocate }\end{array}$ & None & None & None & None & None & None \\
\hline James D. Thomas & $\begin{array}{l}\text { Cleveland Clinic Foundation- } \\
\text { Moore Chair of Cardiovascular } \\
\text { Imaging }\end{array}$ & None & None & None & None & None & None \\
\hline \multicolumn{8}{|c|}{ 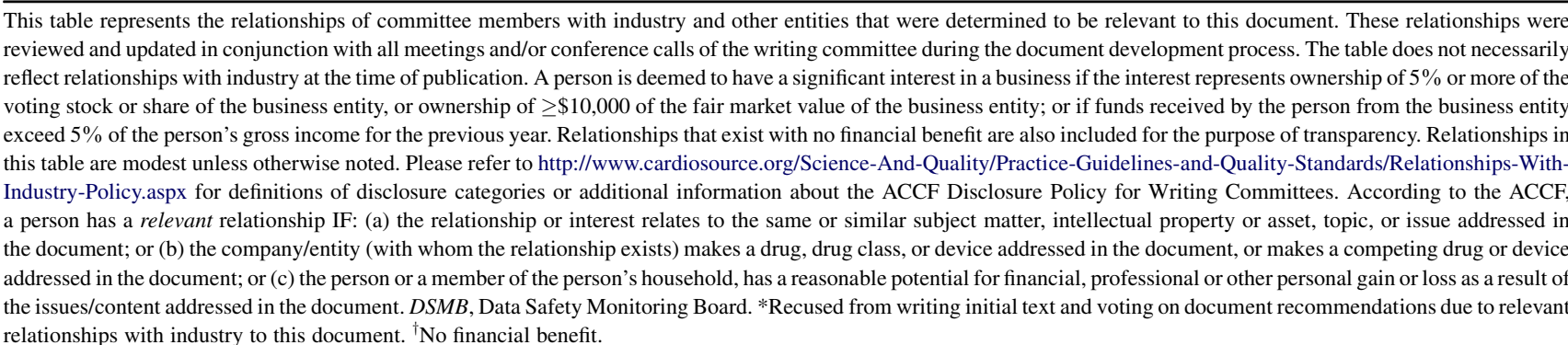 } \\
\hline
\end{tabular}


APPENDIX 2. Reviewer relationships with industry and other entities (relevant)—2012 ACCF/AATS/SCAI/STS expert consensus document on transcatheter aortic valve replacement

\begin{tabular}{|c|c|c|c|c|c|c|c|c|}
\hline Peer reviewer & Representation & Employment & Consultant & $\begin{array}{c}\text { Speaker's } \\
\text { bureau }\end{array}$ & $\begin{array}{l}\text { Ownership/ } \\
\text { partnership/ } \\
\text { principal }\end{array}$ & $\begin{array}{l}\text { Personal } \\
\text { research }\end{array}$ & $\begin{array}{c}\text { Institutional, } \\
\text { organizational, } \\
\text { or other financial } \\
\text { benefit }\end{array}$ & $\begin{array}{l}\text { Expert } \\
\text { witness }\end{array}$ \\
\hline David H. Adams & $\begin{array}{l}\text { Official } \\
\quad \text { Reviewer- } \\
\text { AATS }\end{array}$ & $\begin{array}{l}\text { Mt. Sinai Medical } \\
\text { Center } \\
\text { Department of } \\
\text { Thoracic } \\
\text { Surgery- } \\
\text { Professor and } \\
\text { Chair }\end{array}$ & $\begin{array}{l}\text { - Edwards } \\
\text { Lifesciences* }\end{array}$ & None & None & None & - Medtronic & None \\
\hline Gabriel S. Aldea & $\begin{array}{l}\text { Official } \\
\quad \text { Reviewer- } \\
\text { STS }\end{array}$ & $\begin{array}{l}\text { University of } \\
\text { Washington } \\
\text { Medical } \\
\text { Center- } \\
\text { Associate } \\
\text { Professor of } \\
\text { Medicine }\end{array}$ & None & None & None & None & None & None \\
\hline Ottavio Alfieri & $\begin{array}{l}\text { Organizational } \\
\text { Reviewer- } \\
\text { EACTS }\end{array}$ & $\begin{array}{l}\text { San Raffaele } \\
\text { University } \\
\text { Hospital- } \\
\text { Professor, } \\
\text { Division of } \\
\text { Cardiac Surgery }\end{array}$ & $\begin{array}{l}\text { - Symetis } \\
\text { - Valtech }\end{array}$ & None & None & None & $\begin{array}{l}\text { - Edwards } \\
\text { Lifesciences }\end{array}$ & None \\
\hline Stephen H. Bailey & Content Reviewer & $\begin{array}{l}\text { Allegheny General } \\
\text { Hospital } \\
\text { Department of } \\
\text { Cardiovascular } \\
\text { Thoracic } \\
\text { Surgery }\end{array}$ & None & None & None & None & None & None \\
\hline Deepak L. Bhatt & $\begin{array}{l}\text { Official } \\
\text { Reviewer- } \\
\text { ACCF Task } \\
\text { Force on } \\
\text { Clinical Expert } \\
\text { Consensus } \\
\text { Documents }\end{array}$ & $\begin{array}{l}\text { VA Boston Health } \\
\text { Care System- } \\
\text { Chief, Division } \\
\text { of Cardiology }\end{array}$ & None & None & None & $\begin{array}{l}\text { - Ethicon* } \\
\text { - Medtronic* }\end{array}$ & None & None \\
\hline $\begin{array}{c}\text { Emmanouil } \\
\text { Brilakis }\end{array}$ & $\begin{array}{l}\text { Content } \\
\text { Reviewer- } \\
\text { ACCF } \\
\text { Interventional } \\
\text { Scientific } \\
\text { Council }\end{array}$ & $\begin{array}{l}\text { UT Southwestern } \\
\text { Medical } \\
\text { School- } \\
\text { Director, } \\
\text { Cardiac } \\
\text { Catheterization } \\
\text { Laboratory, VA } \\
\text { North Texas } \\
\text { Health care } \\
\text { System }\end{array}$ & $\begin{array}{l}\text { - St. Jude* } \\
\text { - Terumo }\end{array}$ & None & None & None & $\begin{array}{l}\text { - Abbott Vascular* } \\
\text { - Infraredx* } \\
\text { - Medtronic* }\end{array}$ & None \\
\hline James Burke & $\begin{array}{l}\text { Content } \\
\text { Reviewer- } \\
\text { ACCF } \\
\text { Interventional } \\
\text { Scientific } \\
\text { Council }\end{array}$ & $\begin{array}{l}\text { Lehigh Valley Heart } \\
\text { Specialists }\end{array}$ & None & None & None & None & None & None \\
\hline Shelton Caruthers & $\begin{array}{l}\text { Organizational } \\
\quad \text { Reviewer- } \\
\text { SCMR }\end{array}$ & $\begin{array}{l}\text { Washington } \\
\text { University } \\
\text { School of } \\
\text { Medicine, } \\
\text { Cardiovascular } \\
\text { MR Labs }\end{array}$ & None & None & $\begin{array}{l}\text { - General Electric } \\
\text { - Royal Philips } \\
\text { Electronics }\end{array}$ & - Kereos* & None & None \\
\hline
\end{tabular}




\begin{tabular}{|c|c|c|c|c|c|c|c|c|}
\hline Peer reviewer & Representation & Employment & Consultant & $\begin{array}{c}\text { Speaker's } \\
\text { bureau }\end{array}$ & $\begin{array}{c}\text { Ownership/ } \\
\text { partnership/ } \\
\text { principal }\end{array}$ & $\begin{array}{l}\text { Personal } \\
\text { research }\end{array}$ & $\begin{array}{c}\text { Institutional, } \\
\text { organizational, } \\
\text { or other financial } \\
\text { benefit } \\
\end{array}$ & $\begin{array}{l}\text { Expert } \\
\text { witness }\end{array}$ \\
\hline Albert T. Cheung & $\begin{array}{l}\text { Organizational } \\
\text { Reviewer- } \\
\text { SCA }\end{array}$ & $\begin{array}{l}\text { Hospital of the } \\
\text { University of } \\
\text { Pennsylvania- } \\
\text { Professor of } \\
\text { Anesthesiology } \\
\text { \& Critical Care }\end{array}$ & None & None & None & None & None & None \\
\hline Joseph Cleveland & $\begin{array}{l}\text { Content } \\
\text { Reviewer- } \\
\text { ACCF Council } \\
\text { on } \\
\text { Cardiovascular } \\
\text { Care for Older } \\
\text { Adults }\end{array}$ & $\begin{array}{l}\text { University of } \\
\text { Colorado } \\
\text { Denver- } \\
\text { Associate } \\
\text { Professor }\end{array}$ & $\begin{array}{l}\text { - Baxter } \\
\text { Biosurgery }\end{array}$ & None & None & None & None & None \\
\hline George Dangas & $\begin{array}{l}\text { Content } \\
\quad \text { Reviewer- } \\
\text { ACCF } \\
\text { Interventional } \\
\text { Scientific } \\
\text { Council }\end{array}$ & $\begin{array}{l}\text { Mount Sinai } \\
\text { Medical } \\
\text { Center- } \\
\text { Program } \\
\text { Director, } \\
\text { Interventional } \\
\text { Cardiology }\end{array}$ & $\begin{array}{l}\text { - Abbott } \\
\text { - Boston Scientific } \\
\text { - Bracco } \\
\text { - Guerbet } \\
\text { - Maquet/ } \\
\text { - Datascope } \\
\text { - Medtronic } \\
\text { - St. Jude Medical }\end{array}$ & $\begin{array}{l}\text { - Astra } \\
\text { Zeneca } \\
\text { - NCME }\end{array}$ & None & None & $\begin{array}{l}\text { - Abbott Vascular } \dagger \\
\text { - Lutonix } \dagger \\
\text { - Medtronic } \dagger \\
\text { - Ortho McNeil }\end{array}$ & $\begin{array}{l}\text { Plaintiff, } \\
\quad \text { stroke, } 2011\end{array}$ \\
\hline Larry Dean & $\begin{array}{l}\text { Content } \\
\text { Reviewer- } \\
\text { ACCF } \\
\text { Interventional } \\
\text { Scientific } \\
\text { Council }\end{array}$ & $\begin{array}{l}\text { University of } \\
\text { Washington } \\
\text { School of } \\
\text { Medicine- } \\
\text { Professor of } \\
\text { Medicine \& } \\
\text { Surgery }\end{array}$ & $\begin{array}{l}\text { - Emageon } \\
\text { - Philips Medical }\end{array}$ & $\begin{array}{l}\text { - Edwards } \\
\text { Lifesciences }\end{array}$ & None & None & None & None \\
\hline Pamela Douglas & $\begin{array}{l}\text { Content } \\
\text { Reviewer- } \\
\text { ACCF Imaging } \\
\text { Council }\end{array}$ & $\begin{array}{l}\text { Duke University } \\
\text { Medical } \\
\text { Center-Ursula } \\
\text { Geller Professor } \\
\text { of Research in } \\
\text { Cardiovascular } \\
\text { Diseases }\end{array}$ & None & None & None & $\begin{array}{l}\text { - Atritech } \dagger \\
\text { - Edwards } \\
\text { LifeSciences } \dagger \\
\text { - Viacor } \dagger\end{array}$ & None & None \\
\hline Ted Feldman & $\begin{array}{l}\text { Official } \\
\quad \text { Reviewer- } \\
\text { SCAI }\end{array}$ & $\begin{array}{l}\text { Evanston Hospital } \\
\text { Cardiology } \\
\text { Division- } \\
\text { Director, } \\
\text { Cardiac } \\
\text { Catheterization } \\
\text { Lab }\end{array}$ & $\begin{array}{l}\text { - Abbott } \\
\text { - Boston } \\
\text { Scientific } \\
\text { - Edwards } \\
\text { Lifesciences } \\
\text { - W.L. Gore }\end{array}$ & None & None & $\begin{array}{l}\text { - } \text { Abbott } \\
\text { - Boston } \\
\text { Scientific } \dagger \\
\text { - Edwards } \\
\text { - Lifesciences } \dagger \\
\text { - } \text { W.L. Gore } \dagger\end{array}$ & None & None \\
\hline David A. Fullerton & $\begin{array}{l}\text { Official } \\
\quad \text { Reviewer- } \\
\text { STS \& TVRR } \\
\text { Writing } \\
\text { Committee }\end{array}$ & $\begin{array}{l}\text { University of } \\
\text { Colorado School } \\
\text { of Medicine- } \\
\text { Head, } \\
\text { Cardiothoracic } \\
\text { Surgery }\end{array}$ & None & None & None & None & None & None \\
\hline Mario J. Garcia & $\begin{array}{l}\text { Content } \\
\text { Reviewer- } \\
\text { ACCF Task } \\
\text { Force on } \\
\text { Clinical Expert } \\
\text { Consensus } \\
\text { Documents }\end{array}$ & $\begin{array}{l}\text { Montefiore Medical } \\
\text { Center-Albert } \\
\text { Einstein College } \\
\text { of Medicine- } \\
\text { Chief, Division } \\
\text { of Cardiology }\end{array}$ & - MD Imaging & None & - Pfizer & None & None & None \\
\hline
\end{tabular}




\begin{tabular}{|c|c|c|c|c|c|c|c|c|}
\hline Peer reviewer & Representation & Employment & Consultant & $\begin{array}{c}\text { Speaker's } \\
\text { bureau }\end{array}$ & $\begin{array}{c}\text { Ownership/ } \\
\text { partnership/ } \\
\text { principal }\end{array}$ & $\begin{array}{l}\text { Personal } \\
\text { research }\end{array}$ & $\begin{array}{c}\text { Institutional, } \\
\text { organizational, } \\
\text { or other financial } \\
\text { benefit }\end{array}$ & $\begin{array}{l}\text { Expert } \\
\text { witness }\end{array}$ \\
\hline Frederico Gentile & $\begin{array}{l}\text { Content } \\
\text { Reviewer- } \\
\text { ACCF Task } \\
\text { Force on } \\
\text { Clinical Expert } \\
\text { Consensus } \\
\text { Documents }\end{array}$ & $\begin{array}{r}\text { Centro Medico } \\
\text { Diagnostico }\end{array}$ & None & None & None & None & None & None \\
\hline Robert Guyton & $\begin{array}{l}\text { Content } \\
\text { Reviewer- } \\
\text { ACCF Patient- } \\
\text { Centered Care } \\
\text { Committee }\end{array}$ & $\begin{array}{l}\text { Emory Clinic, } \\
\text { Inc.-Professor } \\
\text { and Chief, } \\
\text { Division of } \\
\text { Cardiothoracic } \\
\text { Surgery }\end{array}$ & None & None & None & $\mathrm{NIH} \dagger$ & None & $\begin{array}{l}\text { Defendant, } \\
\text { Cardiac } \\
\text { Surgery, } 2011\end{array}$ \\
\hline Rebecca T. Hahn & $\begin{array}{l}\text { Organizational } \\
\quad \text { Reviewer-ASE }\end{array}$ & $\begin{array}{l}\text { Columbia } \\
\text { University } \\
\text { College of } \\
\text { Physicians \& } \\
\text { Surgeons } \\
\text {-Associate } \\
\text { Professor of } \\
\text { Clinical } \\
\text { Medicine }\end{array}$ & None & None & None & None & None & None \\
\hline Joerg Hausleiter & $\begin{array}{l}\text { Organizational } \\
\quad \text { Reviewer- } \\
\text { SCCT }\end{array}$ & $\begin{array}{l}\text { Duetsches } \\
\text { Herzzentrum } \\
\text { Munchen }\end{array}$ & None & - Abbott & None & None & None & None \\
\hline Joerg Herrmann & $\begin{array}{l}\text { Content } \\
\text { Reviewer- } \\
\text { ACCF } \\
\text { Interventional } \\
\text { Scientific } \\
\text { Council }\end{array}$ & Mayo Clinic & None & None & None & None & None & None \\
\hline Loren Hirtazka & $\begin{array}{l}\text { Organizational } \\
\text { Reviewer- } \\
\text { AHA }\end{array}$ & $\begin{array}{l}\text { Cardiac, Vascular } \\
\text { and Thoracic } \\
\text { Surgeons, Inc.- } \\
\text { Medical } \\
\text { Director, } \\
\text { Cardiac Surgery }\end{array}$ & None & None & None & None & None & $\begin{array}{l}\text { Defendant, } \\
\text { delayed } \\
\text { pericardial } \\
\text { tamponade } \\
\text { after aortic } \\
\text { valve } \\
\text { surgery, } 2004\end{array}$ \\
\hline Philippe H. Kolh & $\begin{array}{l}\text { Organizational } \\
\quad \text { Reviewer- } \\
\text { EACTS }\end{array}$ & Not reported & Not reported & Not reported & Not reported & Not reported & Not reported & Not reported \\
\hline $\begin{array}{l}\text { Michael J. } \\
\quad \text { Landzberg }\end{array}$ & $\begin{array}{l}\text { Content } \\
\text { Reviewer- } \\
\text { ACCF Adult } \\
\text { Congenital \& } \\
\text { Pediatric } \\
\text { Cardiology } \\
\text { Council }\end{array}$ & $\begin{array}{l}\text { BACH Pulmonary } \\
\text { Hypertension } \\
\text { Serv—Medical } \\
\text { Director, Boston } \\
\text { Adult } \\
\text { Congenital } \\
\text { Heart }\end{array}$ & None & None & None & None & None & None \\
\hline $\begin{array}{l}\text { Catherine Case } \\
\text { Larson }\end{array}$ & $\begin{array}{l}\text { Organizational } \\
\quad \text { Reviewer- } \\
\text { Mended Hearts }\end{array}$ & Not reported & Not reported & Not reported & Not reported & Not reported & Not reported & Not reported \\
\hline Jonathon Leipsic & $\begin{array}{l}\text { Organizational } \\
\quad \text { Reviewer- } \\
\text { SCCT }\end{array}$ & $\begin{array}{l}\text { Providence Health } \\
\text { Care; University } \\
\text { of British } \\
\text { Columbia- } \\
\text { Chairman, } \\
\text { Department of } \\
\text { Radiology; } \\
\text { Assistant } \\
\text { Professor of } \\
\text { Radiology }\end{array}$ & None & $\begin{array}{l}\text { - Edwards } \\
\text { Lifesciences } \\
\text { - GE Healthcare }\end{array}$ & None & None & $\begin{array}{l}\text { - Edwards } \\
\text { Lifesciences* }\end{array}$ & None \\
\hline
\end{tabular}




\begin{tabular}{|c|c|c|c|c|c|c|c|c|}
\hline Peer reviewer & Representation & Employment & Consultant & $\begin{array}{c}\text { Speaker's } \\
\text { bureau }\end{array}$ & $\begin{array}{c}\text { Ownership/ } \\
\text { partnership/ } \\
\text { principal }\end{array}$ & $\begin{array}{l}\text { Personal } \\
\text { research }\end{array}$ & $\begin{array}{c}\text { Institutional, } \\
\text { organizational, } \\
\text { or other financial } \\
\text { benefit } \\
\end{array}$ & $\begin{array}{l}\text { Expert } \\
\text { witness }\end{array}$ \\
\hline Glenn Levine & $\begin{array}{l}\text { Content } \\
\text { Reviewer- } \\
\text { ACC/AHA } \\
\text { Percutaneous } \\
\text { Coronary } \\
\text { Interventions } \\
\text { Guidelines }\end{array}$ & $\begin{array}{l}\text { Baylor College of } \\
\text { Medicine- } \\
\text { Professor of } \\
\text { Medicine }\end{array}$ & None & None & None & None & None & $\begin{array}{l}\text { Defendant, } \\
\quad \text { Patient } \\
\text { non-responsive } \\
\text { after } \\
\text { noncardiac } \\
\text { surgery, } 2010\end{array}$ \\
\hline $\begin{array}{l}\text { Jane A. } \\
\text { Linderbaum }\end{array}$ & $\begin{array}{l}\text { Content } \\
\text { Reviewer- } \\
\text { ACCF Task } \\
\text { Force on } \\
\text { Clinical Expert } \\
\text { Consensus } \\
\text { Documents }\end{array}$ & $\begin{array}{l}\text { Mayo Clinic; } \\
\text { Rochester Saint } \\
\text { Mary's } \\
\text { Hospital- } \\
\text { Assistant } \\
\text { Professor of } \\
\text { Medicine; } \\
\text { Director, CV } \\
\text { Inpatient } \\
\text { Practice }\end{array}$ & None & None & None & None & None & None \\
\hline Ehtisham Mahmud & $\begin{array}{l}\text { Content } \\
\text { Reviewer- } \\
\text { ACCF } \\
\text { Interventional } \\
\text { Scientific } \\
\text { Council }\end{array}$ & $\begin{array}{l}\text { University of } \\
\text { California, San } \\
\text { Diego-- } \\
\text { Professor of } \\
\text { Medicine and } \\
\text { Director, } \\
\text { Cardiac } \\
\text { Catheterization } \\
\text { Laboratory }\end{array}$ & $\begin{array}{l}\text { - Phillips } \\
\text { Medical }\end{array}$ & - Medtronic & None & $\begin{array}{l}\text { - Abbott } \\
\text { Vascular* } \\
\text { - Boston } \\
\text { Scientific* }\end{array}$ & $\begin{array}{l}\text { - St. Jude's } \\
\text { Medical }\end{array}$ & None \\
\hline Margo B. Minissian & $\begin{array}{l}\text { Content } \\
\quad \text { Reviewer- } \\
\text { ACCF } \\
\text { Cardiovascular } \\
\text { Team Council }\end{array}$ & $\begin{array}{l}\text { Cedars Sinai Heart } \\
\text { Institute } \\
\text { Women's Heart } \\
\text { Center; UCLA } \\
\text { School of } \\
\text { Nursing- } \\
\text { Cardiology } \\
\text { Nurse } \\
\text { Practitioner; } \\
\text { Assistant } \\
\text { Clinical } \\
\text { Professor }\end{array}$ & None & None & None & None & None & None \\
\hline David J. Moliterno & $\begin{array}{l}\text { Content } \\
\text { Reviewer- } \\
\text { ACCF Task } \\
\text { Force on } \\
\text { Clinical Expert } \\
\text { Consensus } \\
\text { Documents }\end{array}$ & $\begin{array}{l}\text { University of } \\
\text { Kentucky- } \\
\text { Chief of } \\
\text { Medicine }\end{array}$ & $\begin{array}{l}\text { - Boston } \\
\text { Scientific } \\
\text { - Symetis }\end{array}$ & None & None & None & None & None \\
\hline Srihari Naidu & $\begin{array}{l}\text { Content } \\
\text { Reviewer- } \\
\text { ACCF } \\
\text { Interventional } \\
\text { Scientific } \\
\text { Council }\end{array}$ & $\begin{array}{l}\text { Winthrop } \\
\text { University } \\
\text { Hospital- } \\
\text { Director, } \\
\text { Cardiac Cath } \\
\text { Lab }\end{array}$ & $\begin{array}{l}\text { - Abbott } \\
\text { Vascular }\end{array}$ & None & None & None & None & None \\
\hline Hani Najm & $\begin{array}{l}\text { Content } \\
\quad \text { Reviewer- } \\
\text { ACCF Surgeon } \\
\text { Scientific } \\
\text { Council }\end{array}$ & $\begin{array}{l}\text { National Guard } \\
\text { Health Affairs- } \\
\text { President, Saudi } \\
\text { Heart } \\
\text { Association }\end{array}$ & None & None & None & None & None & None \\
\hline
\end{tabular}




\begin{tabular}{|c|c|c|c|c|c|c|c|c|}
\hline Peer reviewer & Representation & Employment & Consultant & $\begin{array}{c}\text { Speaker's } \\
\text { bureau }\end{array}$ & $\begin{array}{c}\text { Ownership/ } \\
\text { partnership/ } \\
\text { principal }\end{array}$ & $\begin{array}{l}\text { Personal } \\
\text { research }\end{array}$ & $\begin{array}{c}\text { Institutional, } \\
\text { organizational, } \\
\text { or other financial } \\
\text { benefit }\end{array}$ & $\begin{array}{l}\text { Expert } \\
\text { witness }\end{array}$ \\
\hline Rick Nishimura & $\begin{array}{l}\text { Content } \\
\text { Reviewer- } \\
\text { ACC/AHA } \\
\text { Valvular Heart } \\
\text { Disease } \\
\text { Guidelines }\end{array}$ & $\begin{array}{l}\text { Mayo Clinic, } \\
\text { Division of } \\
\text { Cardiovascular } \\
\text { Disease—Judd } \\
\text { and Mary Morris } \\
\text { Leighton } \\
\text { Professor of } \\
\text { Medicine }\end{array}$ & None & None & None & None & None & None \\
\hline Amit R. Patel & $\begin{array}{l}\text { Organizational } \\
\quad \text { Reviewer- } \\
\text { SCMR }\end{array}$ & $\begin{array}{l}\text { Chicago } \\
\quad \text { Department of } \\
\text { Medicine- } \\
\text { Assistant } \\
\text { Professor of } \\
\text { Medicine }\end{array}$ & None & None & None & None & None & None \\
\hline Stephen Ramee & $\begin{array}{l}\text { Official } \\
\text { Reviewer- } \\
\text { ACC Board of } \\
\text { Governors }\end{array}$ & $\begin{array}{l}\text { Ochsner Clinic } \\
\text { Foundation- } \\
\text { Director, } \\
\text { Cardiac } \\
\text { Catheterization }\end{array}$ & None & None & $\begin{array}{l}\text { - Access } \\
\text { Closure* } \\
\text { - Boston } \\
\text { Scientific* } \\
\text { - Hot Spur* }\end{array}$ & $\begin{array}{l}\text { - } \text { Abbott } \dagger \\
\text { - } \text { Boston } \\
\text { Scientific } \dagger \\
\text { - Edwards } \\
\text { - } \text { Lifesciences } \dagger^{\dagger} \text { Medtronic } \dagger\end{array}$ & None & None \\
\hline $\begin{array}{l}\text { Pasala } \\
\quad \text { Ravichandran }\end{array}$ & $\begin{array}{l}\text { Content } \\
\text { Reviewer- } \\
\text { ACCF Surgeon } \\
\text { Scientific } \\
\text { Council \& } \\
\text { ACCF Heart } \\
\text { Failure \& } \\
\text { Transplant } \\
\text { Committee }\end{array}$ & $\begin{array}{l}\text { Oregon Health \& } \\
\text { Science } \\
\text { University- } \\
\text { Associate } \\
\text { Professor }\end{array}$ & None & None & None & None & None & $\begin{array}{r}\text { None } \\
11\end{array}$ \\
\hline Jack Shanewise & $\begin{array}{l}\text { Organizational } \\
\quad \text { Reviewer-SCA }\end{array}$ & $\begin{array}{l}\text { Columbia } \\
\quad \text { University } \\
\text { College of } \\
\text { Physicians \& } \\
\text { Surgeons- } \\
\text { Professor of } \\
\text { Clinical } \\
\text { Anesthesiology }\end{array}$ & None & None & None & None & None & $\begin{array}{l}\text { Defendant, } \\
\text { ruptured } \\
\text { pulmonary } \\
\text { artery by } \\
\text { PA cath, } \\
2011 \\
\text { Defendant, air } \\
\text { embolism } \\
\text { during } \\
\text { heart } \\
\text { surgery, 2010 } \\
\text { Plaintiff, failed } \\
\text { endotracheal } \\
\text { tube exchange, } \\
2011\end{array}$ \\
\hline William J. Stewart & $\begin{array}{l}\text { Organizational } \\
\quad \text { Reviewer-ASE }\end{array}$ & $\begin{array}{c}\text { Cleveland Clinic } \\
\text { Foundation- } \\
\text { Professor of } \\
\text { Medicine }\end{array}$ & None & None & None & None & $\begin{array}{l}\text { - Edwards } \\
\text { Lifesciences } \dagger\end{array}$ & None \\
\hline Lars G. Svensson & $\begin{array}{l}\text { Official } \\
\quad \text { Reviewer- } \\
\text { AATS }\end{array}$ & $\begin{array}{l}\text { Cleveland Clinic } \\
\text { Foundation } \\
\text { Department of } \\
\text { Thoracic \& } \\
\text { Cardiovascular } \\
\text { Surgery- } \\
\text { Director, Center } \\
\text { for Aortic } \\
\text { Surgery }\end{array}$ & None & None & $\begin{array}{l}\text { - Valve Exchange } \\
\text { Cardiosolutions } \\
\text { - Postthorax } \dagger\end{array}$ & $\begin{array}{l}\text { - Edwards } \\
\text { Lifesciences } \dagger\end{array}$ & None & None \\
\hline E. Murat Tuzcu & $\begin{array}{l}\text { Official } \\
\text { Reviewer- } \\
\text { ACCF Board of } \\
\text { Trustees }\end{array}$ & $\begin{array}{l}\text { Cleveland Clinic } \\
\text { Foundation- } \\
\text { Professor of } \\
\text { Medicine }\end{array}$ & None & None & None & None & None & None \\
\hline
\end{tabular}


APPENDIX 2. Continued

\begin{tabular}{|c|c|c|c|c|c|c|c|c|}
\hline Peer reviewer & Representation & Employment & Consultant & $\begin{array}{c}\text { Speaker's } \\
\text { bureau }\end{array}$ & $\begin{array}{c}\text { Ownership/ } \\
\text { partnership/ } \\
\text { principal }\end{array}$ & $\begin{array}{l}\text { Personal } \\
\text { research }\end{array}$ & $\begin{array}{c}\text { Institutional, } \\
\text { organizational, } \\
\text { or other financial } \\
\text { benefit }\end{array}$ & $\begin{array}{l}\text { Expert } \\
\text { witness }\end{array}$ \\
\hline Hector Ventura & $\begin{array}{l}\text { Content } \\
\text { Reviewer- } \\
\text { ACCF Heart } \\
\text { Failure \& } \\
\text { Transplant } \\
\text { Committee }\end{array}$ & $\begin{array}{l}\text { Ochsner Clinic } \\
\text { Foundation } \\
\text { Department of } \\
\text { Cardiology- } \\
\text { Director, Section } \\
\text { of Cardiomyo- } \\
\text { pathy and Heart } \\
\text { Transplan-tation }\end{array}$ & None & None & None & $\begin{array}{l}\text { - PARTNER Trial } \\
\text { Executive } \\
\text { Committee } \dagger\end{array}$ & None & None \\
\hline Robert Vincent & $\begin{array}{l}\text { Content } \\
\text { Reviewer- } \\
\text { ACCF Adult } \\
\text { Congenital \& } \\
\text { Pediatric } \\
\text { Cardiology } \\
\text { Council }\end{array}$ & $\begin{array}{l}\text { Children's Sibley } \\
\text { Heart Center- } \\
\text { Co-Medical } \\
\text { Director, Heart } \\
\text { Transplant; } \\
\text { Director, } \\
\text { Cardiac } \\
\text { Catheterization } \\
\text { Laboratory }\end{array}$ & None & None & None & None & $\begin{array}{l}\text { AGA Medical } \\
\text { Corp } \dagger\end{array}$ & $\begin{array}{l}\text { Defendant, vascular } \\
\text { injury during } \\
\text { cath, 2009 } \\
\text { Plaintiff, air } \\
\text { embolus, } 2008 \\
\text { Defendant, cause of } \\
\quad \text { dilated } \\
\text { cardiomyop- } \\
\text { athy, } 2007\end{array}$ \\
\hline $\begin{array}{l}\text { Andrew R. } \\
\text { Weintraub }\end{array}$ & $\begin{array}{l}\text { Organizational } \\
\quad \text { Reviewer- } \\
\text { HFSA }\end{array}$ & $\begin{array}{l}\text { Tufts University } \\
\text { School of } \\
\text { Medicine- } \\
\text { Assistant } \\
\text { Professor of } \\
\text { Medicine }\end{array}$ & None & None & None & None & - Cardiva Inc & None \\
\hline Christopher White & $\begin{array}{l}\text { Content } \\
\text { Reviewer- } \\
\text { ACCF } \\
\text { Interventional } \\
\text { Scientific } \\
\text { Council }\end{array}$ & $\begin{array}{l}\text { Ochsner Clinical } \\
\text { Foundation- } \\
\text { Chairman, } \\
\text { Department of } \\
\text { Cardiology }\end{array}$ & - Baxter & None & None & - St. Jude & None & None \\
\hline Alan Zajarias & $\begin{array}{l}\text { Official } \\
\quad \text { Reviewer- } \\
\text { SCAI }\end{array}$ & $\begin{array}{l}\text { Washington } \\
\text { University } \\
\text { School of } \\
\text { Medicine- } \\
\text { Associate } \\
\text { Professor of } \\
\text { Medicine } \\
\end{array}$ & $\begin{array}{l}\text { - St. Jude's } \\
\text { Medical } \\
\text { - Edwards } \\
\text { Lifesciences }\end{array}$ & None & None & $\begin{array}{l}\text { - Edwards } \\
\text { Lifesciences (PI, } \\
\text { PARTNER } 2 \\
\text { Trial) } \dagger\end{array}$ & None & None \\
\hline
\end{tabular}

This table represents the relationships of reviewers with industry and other entities that were disclosed at the time of peer review and determined to be relevant. It does not necessarily reflect relationships with industry at the time of publication. A person is deemed to have a significant interest in a business if the interest represents ownership of $\geq 5 \%$ of the voting stock or share of the business entity, or ownership of $\geq \$ 10,000$ of the fair market value of the business entity; or if funds received by the person from the business entity exceed $5 \%$ of the person's gross income for the previous year. A relationship is considered to be modest if it is less than significant under the preceding definition. Relationships that exist with no financial benefit are also included for the purpose of transparency. Relationships in this table are modest unless otherwise noted. Names are listed in alphabetical order within each category of review. According to the ACCF/AHA, a person has a relevant relationship IF: (a) the relationship or interest relates to the same or similar subject matter, intellectual property or asset, topic, or issue addressed in the document; or (b) the company/entity (with whom the relationship exists) makes a drug, drug class, or device addressed in the document, or makes a competing drug or device addressed in the document; or (c) the person or a member of the person's household, has a reasonable potential for financial, professional or other personal gain or loss as a result of the issues/content addressed in the document. AATS, American Association for Thoracic Surgery; ACCF, American College of Cardiology Foundation; $A C E$, Accreditation for Cardiovascular Excellence; AHA, American Heart Association; ASE, American Society of Echocardiography; DCRI, Duke Clinical Research Institute; EACTS, European Association for Cardio-Thoracic Surgery; HFSA, Heart Failure Society of America; NCDR-CARE, National Cardiovascular Data Registry-Carotid Artery Revascularization and Endarterectomy; NIH, National Institutes of Health; PARTNER, Placement of Aortic Transcatheter Valve Trial; PI, principal investigator; SCA, Society of Cardiovascular Anesthesiologists; SCAI, Society for Cardiovascular Angiography and Interventions; SCCT, Society of Cardiovascular Computed Tomography; SCMR, Society for Cardiovascular Magnetic Resonance; STS, Society of Thoracic Surgeons. *Significant relationship. †No financial benefit. 